\title{
Irreducible modules over finite simple Lie pseudoalgebras III. Primitive pseudoalgebras of type $H$
}

\author{
Bojko Bakalov $^{\mathrm{a}}$, Alessandro D'Andrea ${ }^{\mathrm{b}, *}$, Victor G. $\mathrm{Kac}^{\mathrm{c}}$ \\ a Department of Mathematics, North Carolina State University, Raleigh, \\ NC 27695, USA \\ b Dipartimento di Matematica, Istituto "Guido Castelnuovo", Università di Roma \\ "La Sapienza", 00185 Rome, Italy \\ c Department of Mathematics, MIT, Cambridge, MA 02139, USA
}

\section{A R T I C L E I N F O}

\section{Article history:}

Received 13 January 2020

Received in revised form 15 July

2021

Accepted 16 July 2021

Available online xxxx

Communicated by Roman

Bezrukavnikov

\section{Keywords:}

Lie pseudoalgebra

Lie-Cartan algebra of vector fields

Hopf algebra

Conformally symplectic geometry

\section{A B S T R A C T}

A Lie conformal algebra is an algebraic structure that encodes the singular part of the operator product expansion of chiral fields in conformal field theory. A Lie pseudoalgebra is a generalization of this structure, for which the algebra of polynomials $\mathbf{k}[\partial]$ in the indeterminate $\partial$ is replaced by the universal enveloping algebra $U(\mathfrak{d})$ of a finite-dimensional Lie algebra $\mathfrak{d}$ over the base field $\mathbf{k}$. The finite (i.e., finitely generated over $U(\mathfrak{d}))$ simple Lie pseudoalgebras were classified in our 2001 paper [1]. The complete list consists of primitive Lie pseudoalgebras of type $W, S, H$, and $K$, and of current Lie pseudoalgebras over them or over simple finite-dimensional Lie algebras. The present paper is the third in our series on representation theory of simple Lie pseudoalgebras. In the first paper, we showed that any finite irreducible module over a primitive Lie pseudoalgebra of type $W$ or $S$ is either an irreducible tensor module or the image of the differential in a member of the pseudo de Rham complex. In the second paper, we established a similar result for primitive Lie pseudoalgebras of type $K$, with the pseudo de Rham complex replaced by a certain reduction, called the contact pseudo de Rham complex. This reduction in the context of contact geometry

\footnotetext{
* Corresponding author.

E-mail addresses: bojko_bakalov@ncsu.edu (B. Bakalov), dandrea@mat.uniroma1.it (A. D'Andrea), kac@math.mit.edu (V.G.Kac).
} 
was discovered by M. Rumin [11]. In the present paper, we show that for primitive Lie pseudoalgebras of type $H$, a similar to type $K$ result holds with the contact pseudo de Rham complex replaced by a suitable complex. However, the type $H$ case in more involved, since the annihilation algebra is not the corresponding Lie-Cartan algebra, as in other cases, but an irreducible central extension. When the action of the center of the annihilation algebra is trivial, this complex is related to work by M. Eastwood [6] on conformally symplectic geometry, and we call it conformally symplectic pseudo de Rham complex.

(C) 2021 Elsevier Inc. All rights reserved.

\section{Contents}

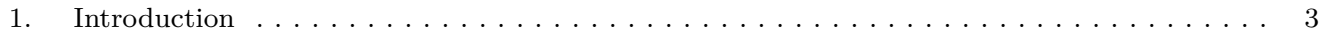

2. Preliminaries on Lie pseudoalgebras $\ldots \ldots \ldots \ldots \ldots \ldots \ldots \ldots \ldots \ldots \ldots \ldots \ldots \ldots \ldots \ldots \ldots \ldots \ldots$

2.1. Bases and filtrations of $H$ and $H^{*} \ldots \ldots \ldots \ldots \ldots \ldots$

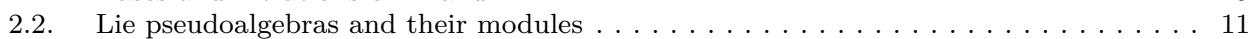

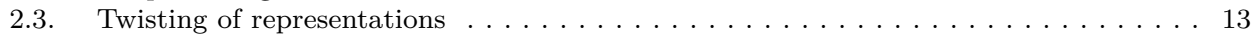

2.4. Annihilation algebras of Lie pseudoalgebras . . . . . . . . . . . . . . . . 14

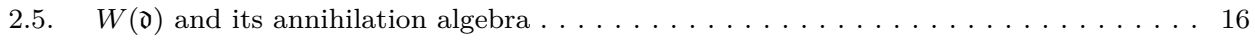

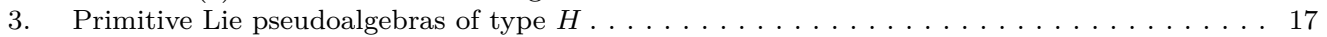

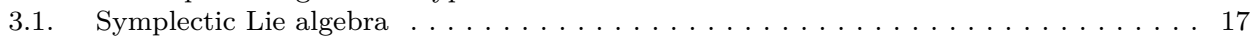

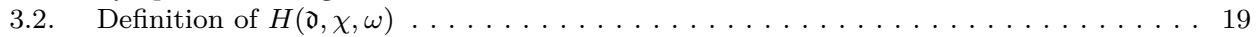

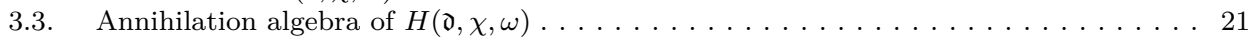

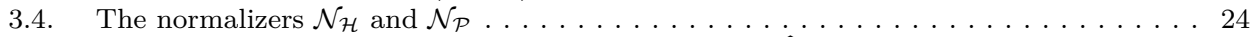

3.5. Finite-dimensional irreducible representations of $\iota_{*}^{-1}(\widehat{\mathfrak{d}}) \ldots \ldots \ldots \ldots$

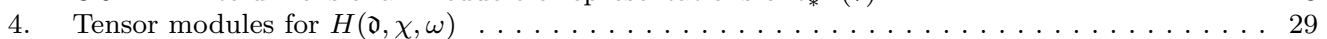

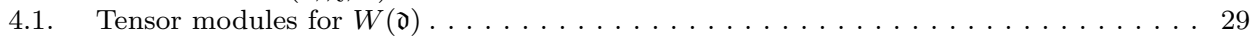

4.2. Restriction of tensor modules from $W(\mathfrak{d})$ to $H(\mathfrak{d}, \chi, \omega) \ldots \ldots \ldots$

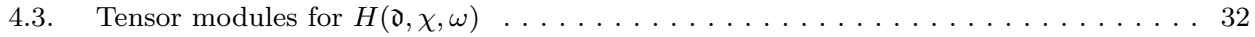

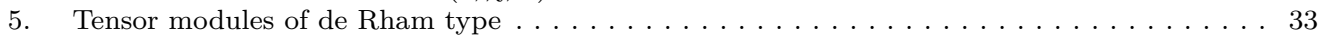

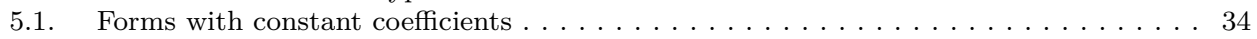

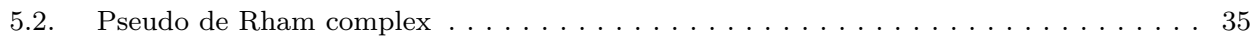

5.3. Conformally symplectic pseudo de Rham complex for $\chi=0 \ldots \ldots \ldots$

5.4. Twisted conformally symplectic pseudo de Rham complex . . . . . . . . . . . . 38

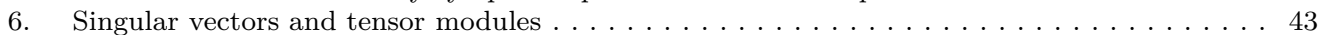

6.1. Conformal representations of $\tilde{\mathcal{P}} \ldots \ldots \ldots \ldots \ldots \ldots$

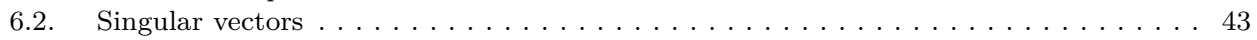

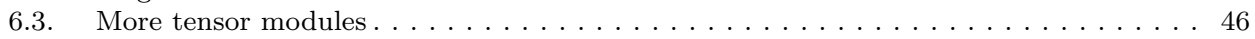

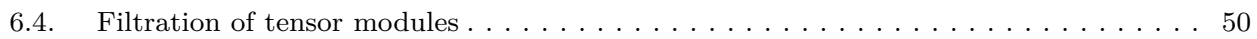

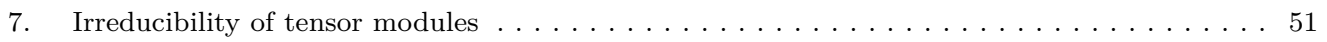

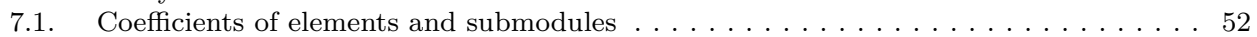

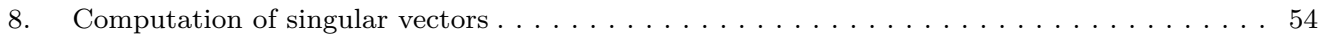

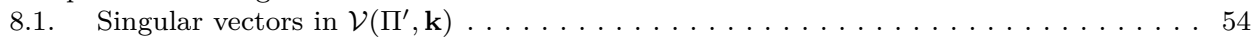

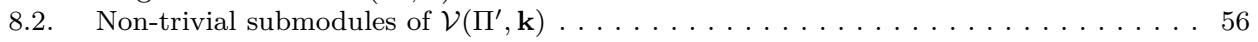

8.3. Singular vectors in $\mathcal{V}\left(\Pi^{\prime}, U\right)$ for a non-trivial $U \ldots \ldots \ldots \ldots \ldots$

8.4. Singular vectors of degree one in $\mathcal{V}\left(\Pi^{\prime}, R\left(\pi_{n}\right)\right), 1 \leq n \leq N \ldots \ldots \ldots$

8.5. Singular vectors of degree two in $\mathcal{V}\left(\Pi^{\prime}, R\left(\pi_{n}\right)\right), 1 \leq n \leq N \ldots \ldots$

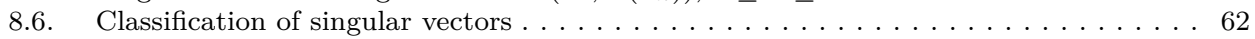

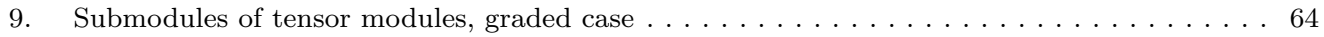

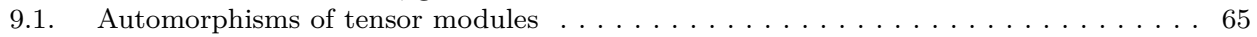

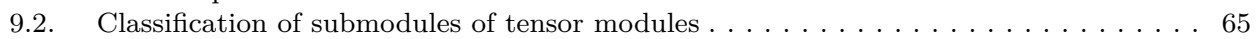

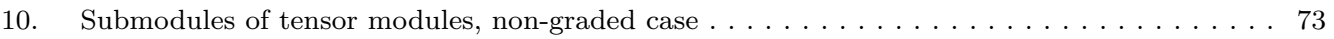


11. Classification of finite irreducible modules over $H(\mathfrak{d}, \chi, \omega) \ldots \ldots \ldots$

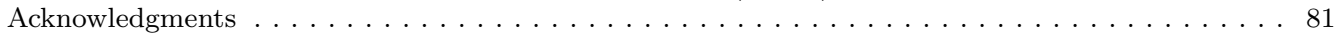

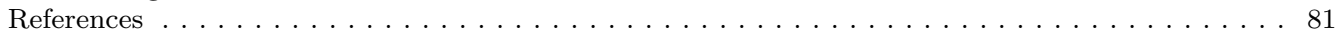

\section{Introduction}

The present paper is the third in our series of papers on representation theory of simple Lie pseudoalgebras, the first two of which are [2] and [3]. As in these papers, we will work over an algebraically closed field $\mathbf{k}$ of characteristic 0 . Unless otherwise specified, all vector spaces, linear maps and tensor products will be considered over $\mathbf{k}$.

Recall that a Lie pseudoalgebra is a (left) module $L$ over a cocommutative Hopf algebra $H$, endowed with a pseudobracket

$$
L \otimes L \rightarrow(H \otimes H) \otimes_{H} L, \quad a \otimes b \mapsto[a * b],
$$

which is an $H$-bilinear map of $H$-modules, satisfying some analogs of the skew-symmetry and Jacobi identity of a Lie algebra bracket (see [4], [1], or (2.21) and (2.22) in Section 2.2 of the present paper).

In the case when $H=\mathbf{k}$, this notion coincides with that of a Lie algebra. Furthermore, any Lie algebra $\mathfrak{g}$ gives rises to a Lie pseudoalgebra Cur $\mathfrak{g}=H \otimes \mathfrak{g}$ over $H$ with the pseudobracket

$$
[(1 \otimes a) *(1 \otimes b)]=(1 \otimes 1) \otimes_{H}[a, b]
$$

extended to the whole Cur $\mathfrak{g}$ by $H$-bilinearity.

In the case where $H=\mathbf{k}[\partial]$, the algebra of polynomials in an indeterminate $\partial$ with the comultiplication $\Delta(\partial)=\partial \otimes 1+1 \otimes \partial$, the notion of a Lie pseudoalgebra coincides with that of a Lie conformal algebra [8]. The main result of [5] states that in this case any finite (i.e., finitely generated over $H=\mathbf{k}[\partial]$ ) simple Lie pseudoalgebra is isomorphic to either Cur $\mathfrak{g}$ with simple finite-dimensional $\mathfrak{g}$, or to the Virasoro pseudoalgebra Vir $=\mathbf{k}[\partial] \ell$, where

$$
[\ell * \ell]=(1 \otimes \partial-\partial \otimes 1) \otimes_{\mathbf{k}[\partial]} \ell
$$

In [1], we generalized this result to the case when $H=U(\mathfrak{d})$, where $\mathfrak{d}$ is a finitedimensional Lie algebra. The generalization of the Virasoro pseudoalgebra is the Lie pseudoalgebra $W(\mathfrak{d})=H \otimes \mathfrak{d}$ with the pseudobracket

$$
\begin{aligned}
{[(1 \otimes a) *(1 \otimes b)]=} & (1 \otimes 1) \otimes_{H}(1 \otimes[a, b]) \\
& +(b \otimes 1) \otimes_{H}(1 \otimes a)-(1 \otimes a) \otimes_{H}(1 \otimes b) .
\end{aligned}
$$


The main result of [1] is that all non-zero subalgebras of the Lie pseudoalgebra $W(\mathfrak{d})$ are simple and non-isomorphic, and along with Cur $\mathfrak{g}$, where $\mathfrak{g}$ is a simple finitedimensional Lie algebra, they provide a complete list of finitely generated over $H$ simple Lie pseudoalgebras. Furthermore, in [1] we gave a description of all subalgebras of $W(\mathfrak{d})$. Namely, a complete list consists of the "primitive" series: the special Lie pseudoalgebras $S(\mathfrak{d}, \chi)$, the Hamiltonian Lie pseudoalgebras $H(\mathfrak{d}, \chi, \omega)$, the contact Lie pseudoalgebras $K(\mathfrak{d}, \theta)$, and their "current" extensions.

The geometric meaning of the data $\chi, \omega$, and $\theta$ is as follows: $\chi \in \mathfrak{d}^{*}$ is a closed 1-form $(=$ trace-form $)$, i.e., $(d \chi)(a \wedge b):=\chi([a, b])=0 ; \omega \in \bigwedge^{2} \mathfrak{d}^{*}$ is a conformally symplectic form, i.e., it is non-degenerate and

$$
d \omega+\chi \wedge \omega=0
$$

finally, $\theta \in \mathfrak{d}^{*}$ is a contact 1 -form (cf. [3]). This explains why the representation theory of the $K$ and $H$ type Lie pseudoalgebras is intimately related to the constructions in contact and conformally symplectic geometry of [11] and [6].

For every Lie pseudoalgebra $L$, we have a functor [1]

$$
Y \mapsto \mathcal{A}_{Y} L:=Y \otimes_{H} L
$$

that assigns a Lie algebra $\mathcal{A}_{Y} L$ to any commutative associative algebra $Y$ equipped with compatible left and right actions of the Hopf algebra $H$. The Lie algebra bracket on $\mathcal{A}_{Y} L$ is given by

$$
\left[x \otimes_{H} a, y \otimes_{H} b\right]=\sum_{i}\left(x f_{i}\right)\left(y g_{i}\right) \otimes_{H} c_{i}, \quad \text { if } \quad[a * b]=\sum_{i}\left(f_{i} \otimes g_{i}\right) \otimes_{H} c_{i} .
$$

The main tool in the study of Lie pseudoalgebras and their representations is the annihilation algebra $\mathcal{A}_{X} L$, where $X=H^{*}$ is the commutative associative algebra dual to the coalgebra $H$. In particular, a module over a Lie pseudoalgebra $L$ is the same as a "conformal" module over the extended annihilation Lie algebra $\mathfrak{d} \ltimes \mathcal{A}_{X} L$ (see [2] and Proposition 2.1 below).

Note that $X \simeq \mathcal{O}_{M}:=\mathbf{k}\left[\left[t^{1}, \ldots, t^{M}\right]\right]$ where $M=\operatorname{dim} \mathfrak{d}$. We define a topology on $\mathcal{O}_{M}$ with a fundamental system of neighborhoods of 0 given by the powers of the maximal ideal $\left(t^{1}, \ldots, t^{M}\right)$. Then the annihilation algebra of the Lie pseudoalgebra $W(\mathfrak{d})$ is isomorphic to the Lie-Cartan algebra $W_{M}$ of continuous derivations of $\mathcal{O}_{M}$ (see [2] and Section 2.5 below). Similar isomorphisms hold for $S$ - and $K$-type Lie pseudoalgebras [2], [3]. However the annihilation algebra of $H(\mathfrak{d}, \chi, \omega)$ is isomorphic to the Lie algebra structure $P_{M}$ on $\mathcal{O}_{M}, M=2 N$, given by

$$
[f, g]=\sum_{i=1}^{N}\left(\frac{\partial f}{\partial t^{i}} \frac{\partial g}{\partial t^{N+i}}-\frac{\partial f}{\partial t^{N+i}} \frac{\partial g}{\partial t^{i}}\right)
$$


so that $P_{M} / \mathbf{k} 1$ is isomorphic to the Hamiltonian Lie-Cartan algebra $H_{2 N}$ of vector fields annihilating the standard symplectic form $\sum_{i=1}^{N} d t^{i} \wedge d t^{N+i}$. This explains the name and notation $H$ of the corresponding Lie pseudoalgebra. We hope that the reader will not confuse this $H$ with the Hopf algebra $H=U(\mathfrak{d})$.

In [2], we constructed all finite (i.e., finitely generated over $H=U(\mathfrak{d})$ ) irreducible modules over the Lie pseudoalgebras $W(\mathfrak{d})$ and $S(\mathfrak{d}, \chi)$. The simplest non-zero module over $W(\mathfrak{d})$ is $\Omega^{0}(\mathfrak{d})=H$, with the action given by

$$
(f \otimes a) * g=-(f \otimes g a) \otimes_{H} 1, \quad f, g \in H, a \in \mathfrak{d} .
$$

A generalization of this construction is as follows [2]. Given a finite-dimensional $\mathfrak{g l} \mathfrak{d}$ module $V_{0}$, we construct a representation of the Lie pseudoalgebra $W(\mathfrak{d})$ on the $H$ module $\mathcal{T}\left(V_{0}\right)=H \otimes V_{0}$ by

$$
\begin{aligned}
\left(1 \otimes \partial_{i}\right) *(1 \otimes v)= & (1 \otimes 1) \otimes_{H}\left(1 \otimes\left(\operatorname{ad} \partial_{i}\right) v\right)-\left(1 \otimes \partial_{i}\right) \otimes_{H}(1 \otimes v) \\
& +\sum_{j=1}^{N}\left(\partial_{j} \otimes 1\right) \otimes_{H}\left(1 \otimes e_{i}^{j} v\right),
\end{aligned}
$$

where $v \in V_{0},\left\{\partial_{i}\right\}$ is a basis of $\mathfrak{d}$ and elements $e_{i}^{j} \in \mathfrak{g l d}$ satisfy $e_{i}^{j} \partial_{k}=\delta_{k}^{j} \partial_{i}$. Such representations may be twisted with a $\mathfrak{d}$-module $\Pi$, by setting up on the $H$-module $\mathcal{T}\left(\Pi, V_{0}\right)=H \otimes\left(\Pi \otimes V_{0}\right)$ a $W(\mathfrak{d})$-action obtained by adding the term $(1 \otimes 1) \otimes_{H}\left(1 \otimes \partial_{i} v\right)$ to the right-hand side of (1.2). All of the above modules are called tensor $W(\mathfrak{d})$-modules. A more conceptual construction of tensor $W(\mathfrak{d})$-modules may be found in [2, Section 4].

The main result of [2] states that any finite irreducible $W(\mathfrak{d})$-module is a unique quotient of a tensor $W(\mathfrak{d})$-module $\mathcal{T}\left(\Pi, V_{0}\right)$ for some finite-dimensional irreducible modules $\Pi$ and $V_{0}$ over $\mathfrak{d}$ and $\mathfrak{g l} \mathfrak{d}$, respectively. Furthermore, [2] describes all cases where $\mathcal{T}\left(\Pi, V_{0}\right)$ are not irreducible, and provides an explicit construction of their irreducible quotients, called the degenerate $W(\mathfrak{d})$-modules. Namely, we prove that all degenerate $W(\mathfrak{d})$-modules occur as images of the differential $\mathrm{d}$ in the $\Pi$-twisted pseudo de Rham complex of $W(\mathfrak{d})$-modules

$$
0 \rightarrow \Omega_{\Pi}^{0}(\mathfrak{d}) \stackrel{\mathrm{d}}{\rightarrow} \Omega_{\Pi}^{1} \stackrel{\mathrm{d}}{\rightarrow} \cdots \stackrel{\mathrm{d}}{\rightarrow} \Omega_{\Pi}^{\operatorname{dim} \mathfrak{d}}(\mathfrak{d})
$$

where $\Omega_{\Pi}^{n}(\mathfrak{d})=\mathcal{T}\left(\Pi, \bigwedge^{n} \mathfrak{d}^{*}\right)$ is the space of pseudo $n$-forms.

In the present paper, we construct all finite irreducible modules over the Hamiltonian Lie pseudoalgebra $H(\mathfrak{d}, \chi, \omega)$. This Lie pseudoalgebra is constructed as follows. Choose a basis $\left\{\partial_{i}\right\}_{i=1}^{2 N}$ of $\mathfrak{d}$, and the dual basis $\left\{\partial^{i}\right\}_{i=1}^{2 N}$ with respect to the (non-degenerate) bilinear form $\omega$ on $\mathfrak{d}$, so that $\omega\left(\partial^{i} \wedge \partial_{j}\right)=\delta_{j}^{i}$. Let

$$
r=\sum_{i=1}^{2 N} \partial_{i} \otimes \partial^{i}=-\sum_{i=1}^{2 N} \partial^{i} \otimes \partial_{i}=\sum_{i, j=1}^{2 N} r^{i j} \partial_{i} \otimes \partial_{j}
$$


and define $s \in \mathfrak{d}$ by

$$
\chi(a)=\omega(s \wedge a), \quad a \in \mathfrak{d} .
$$

Let, as before, $H=U(\mathfrak{d})$ and consider the free $H$-module of rank $1, H e$, equipped with the pseudobracket given by

$$
[e * e]=(r+s \otimes 1-1 \otimes s) \otimes_{H} e,
$$

and extended to $H e$ by bilinearity. This is a simple Lie pseudoalgebra, denoted by $H(\mathfrak{d}, \chi, \omega)$. There is a unique pseudoalgebra embedding of $H(\mathfrak{d}, \chi, \omega)$ in $W(\mathfrak{d})=H \otimes \mathfrak{d}$, defined by [1]

$$
e \mapsto-r+1 \otimes s
$$

We will denote again by $e$ its image in $W(\mathfrak{d})$.

Let $\mathfrak{s p} \mathfrak{d}$ be the symplectic subalgebra of the Lie algebra $\mathfrak{g l} \mathfrak{d}$, defined by the skewsymmetric bilinear form $\omega$. Let $\left\{e_{j}^{i}\right\} \subset$ End $\mathfrak{d}$ be the basis of matrix units in the basis $\left\{\partial_{i}\right\}$ of $\mathfrak{d}$, and let $e^{i j}=\sum_{k} r^{i k} e_{k}^{j}$ be another basis of End $\mathfrak{d}$, where the $r^{i k}$ are defined by (1.4). Then the elements

$$
f^{i j}=-\frac{1}{2}\left(e^{i j}+e^{j i}\right), \quad 1 \leq i \leq j \leq 2 N,
$$

form a basis of $\mathfrak{s p} \mathfrak{d}$.

It is important to highlight that repeating the same strategy as in primitive Lie pseudoalgebras of type $W, S, K$ is not straightforward, as in type $H$ there are several issues that force novel and more irregular behavior.

- The annihilation algebra $\mathcal{P}$ of $H(\mathfrak{d}, \chi, \omega)$ is a graded Lie algebra with a non-trivial one-dimensional center. The above embedding of $H(\mathfrak{d}, \chi, \omega)$ in $W(\mathfrak{d})$ does not induce a corresponding embedding of annihilation algebras, since the center of $\mathcal{P}$ lies in its kernel; as a consequence, a complete family of tensor modules for $H(\mathfrak{d}, \chi, \omega)$ cannot be obtained by restriction from tensor modules for $W(\mathfrak{d})$, as one will only obtain modules where the center acts trivially.

- Constructing tensor modules as induced modules does not yield, as with primitive pseudoalgebras of other types, a corresponding grading, but only a filtration. Indeed, even though the annihilation algebra $\mathcal{P}$ is graded, central elements of $\mathcal{P}$ lie in degree -2 , whereas Schur's Lemma forces them to act via scalar multiplication which, if homogeneous, should have degree 0 .

- The proof of existence of a unique maximal submodule in degenerate tensor modules fails, as it relies on a grading. In principle, non-constant singular vectors (i.e., of positive degree in the filtration) may generate elements of lower degree and they indeed do so in explicit examples. 
- Finally, non-constant singular vectors in degenerate tensor $H(\mathfrak{d}, \chi, \omega)$-modules split in multiple isotypical $\mathfrak{s p} \mathfrak{d}$-components. This hints towards a more complicated structure of the lattice of their submodules and the possibility, which indeed occurs, that an irreducible quotient of each tensor module contains more non-isomorphic $\mathfrak{s p d}$ summands of singular vectors.

All issues, but the last one, disappear if one only focuses on $H(\mathfrak{d}, \chi, \omega)$-modules with a trivial action of the center, i.e., when the action of $\mathcal{P} \simeq P_{2 N}$ factors through $\mathcal{H} \simeq H_{2 N}$, and the usual strategy may be employed. In such case, writing down the action of $e$ in a tensor $W(\mathfrak{d})$-module $\mathcal{T}\left(V_{0}\right)$, where $V_{0}$ is a $\mathfrak{d} \oplus \mathfrak{g l} \mathfrak{d}$-module, and suitably twisting by $\chi$, we obtain a formula for the $H(\mathfrak{d}, \chi, \omega)$-module $\mathcal{T}\left(V_{0}\right)=H \otimes V_{0}$, where now $V_{0}$ is a $\mathfrak{d} \oplus \mathfrak{s p} \mathfrak{d}$-module. Explicitly, we have for $v \in V_{0}=\mathbf{k} \otimes V_{0} \subset \mathcal{T}\left(V_{0}\right)$ :

$$
\begin{aligned}
& e * v=\sum_{k=1}^{2 N}\left(\bar{\partial}_{k} \otimes \partial^{k}\right) \otimes_{H} v-\sum_{k=1}^{2 N}\left(\bar{\partial}_{k} \otimes 1\right) \otimes_{H}\left(\partial^{k}+\operatorname{ad}^{\mathfrak{s p}} \partial^{k}\right) v \\
& +\sum_{i, j=1}^{2 N}\left(\bar{\partial}_{i} \bar{\partial}_{j} \otimes 1\right) \otimes_{H} f^{i j} v
\end{aligned}
$$

where $\bar{\partial}_{i}=\partial_{i}-\chi\left(\partial_{i}\right)$, and $\operatorname{ad}^{\mathfrak{s p}} \partial^{k}$ is the image of ad $\partial^{k}+\partial^{k} \otimes \chi$ under the projection $\mathfrak{g l} \mathfrak{d} \rightarrow \mathfrak{s p} \mathfrak{d}$ defined by $e^{i j} \mapsto-f^{i j}$.

Theorem 6.1 of the present paper, analogous to those in [2] and [3], implies that every finite irreducible $H(\mathfrak{d}, \chi, \omega)$-module, with a trivial action of the center of $\mathcal{P}$, is a quotient of the tensor module $\mathcal{T}\left(V_{0}\right)$, where $V_{0}$ is a finite-dimensional irreducible $\mathfrak{d} \oplus \mathfrak{s p} \mathfrak{d}$-module. We describe all cases where the $H(\mathfrak{d}, \chi, \omega)$-modules $\mathcal{T}\left(V_{0}\right)$ are not irreducible and give an explicit construction of their irreducible quotients, called degenerate $H(\mathfrak{d}, \chi, \omega)$-modules.

It turns out that, in analogy with the contact case [3], all of the above non-trivial degenerate $H(\mathfrak{d}, \chi, \omega)$-modules appear as images of composition of two maps (not one as in the contact case) in a certain complex of $H(\mathfrak{d}, \chi, \omega)$-modules, which we call the twisted conformally symplectic pseudo de Rham complex. This complex is constructed in Section 5 by a certain reduction of the pseudo de Rham complex (1.3) (see Theorem 5.1 and Theorem 10.1). The idea of this construction is similar to Eastwood's reduction of the de Rham complex on a conformally symplectic manifold [6]. The structure of the lattice of submodules of members of the twisted conformally symplectic pseudo de Rham complex requires a detailed study of singular vectors. This is carried out in Section 8 and 9 .

Let us now proceed to the case of $H(\mathfrak{d}, \chi, \omega)$-modules with a non-trivial action of central elements in the annihilation algebra $\mathcal{P}$. Since $\mathcal{P}$ is a central extension of the corresponding simple Lie-Cartan algebra $H_{2 N}$, an important role in representation theory of the Lie pseudoalgebra $H(\mathfrak{d}, \chi, \omega)$ is played by the extension $\mathfrak{d}^{\prime}=\mathfrak{d}+\mathbf{k} c$ of the Lie algebra $\mathfrak{d}$ by a 1 -dimensional abelian ideal $\mathbf{k} c$, with brackets: 


$$
[\partial, c]^{\prime}=\chi(\partial) c, \quad\left[\partial_{1}, \partial_{2}\right]^{\prime}=\left[\partial_{1}, \partial_{2}\right]+\omega\left(\partial_{1} \wedge \partial_{2}\right) c, \quad \text { where } \partial, \partial_{1}, \partial_{2} \in \mathfrak{d}
$$

One easily checks that the central elements of $\mathcal{P}$ may only act by a non-trivial scalar when $\chi=0$ and $\omega=\mathrm{d} \zeta$ is exact. Lie algebras with a non-degenerate exact 2-form are known as Frobenius Lie algebras (the simplest example being the non-abelian 2dimensional Lie algebra). The central extension $\mathfrak{d}^{\prime}$ then splits as a direct $\operatorname{sum} \mathfrak{d}^{\zeta} \oplus \mathbf{k} c$, where $\mathfrak{d}^{\zeta}$ is a Lie subalgebra isomorphic to $\mathfrak{d}$ which is a complement to the central ideal $\mathbf{k} c$.

In this case, the definition of tensor modules $\mathcal{T}\left(V_{0}\right)$ must be modified as follows:

$$
\begin{aligned}
e * v=\sum_{k=1}^{2 N}\left(\bar{\partial}_{k} \otimes \partial^{k}\right) \otimes_{H} v & -\sum_{k=1}^{2 N}\left(\bar{\partial}_{k} \otimes 1\right) \otimes_{H}\left(\partial^{k}+\operatorname{ad}^{\mathfrak{s p}} \partial^{k}\right) v \\
& +\sum_{i, j=1}^{2 N}\left(\bar{\partial}_{i} \bar{\partial}_{j} \otimes 1\right) \otimes_{H} f^{i j} v+(1 \otimes 1) \otimes_{H} c v
\end{aligned}
$$

where $c \in \mathbf{k}$ provides the scalar action of the center of $\mathcal{P}$. Once again Theorem 6.1 shows that every finite irreducible non-trivial $H(\mathfrak{d}, 0, \mathrm{~d} \zeta)$-module is a quotient of $\mathcal{T}\left(V_{0}\right)$, where $V_{0}$ is a finite-dimensional $\mathfrak{d}^{\prime} \oplus \mathfrak{s p} \mathfrak{d}$-module. The complex of degenerate $H(\mathfrak{d}, 0, \mathrm{~d} \zeta)$-modules is now constructed by hand, by using the canonical maps between tensor modules whose existence is due to the presence of non-constant singular vectors. The resulting complex is (10.1). The pathological behavior we anticipated indeed occurs. Namely:

- each $\mathcal{V}\left(\Pi^{\prime}, R\left(\pi_{n}\right)\right), 1 \leq n \leq N$, contains two maximal submodules;

- each $\operatorname{Im} D_{\Pi^{\prime}}^{n}, 0 \leq n \leq N$, contains two distinct non-isomorphic $\mathfrak{d}^{\prime} \oplus \mathfrak{s p} \mathfrak{d}$-summands of singular vectors, hence it is a quotient of two distinct degenerate tensor modules.

Somewhat surprisingly, one may show that the whole complex is split exact, and that each member of the complex decomposes into the direct sum of its maximal submodules, which are also irreducible: this is a special behavior of tensor modules when $c \neq 0$, which completely falls apart when $c=0$. Thus, every finite irreducible degenerate $H(\mathfrak{d}, 0, \mathrm{~d} \zeta)$ module, which has a non-trivial action as $c \neq 0$, arises as the image of one differential in this complex. This complex still lacks a differential geometrical construction, and its geometrical meaning is as yet unclear.

Irreducibility of tensor modules, not appearing in the above complexes, is proved in Section 7 (see Theorem 7.1). The resulting complete non-redundant list of finite irreducible $H(\mathfrak{d}, \chi, \omega)$-modules is given in Theorem 11.1.

As a corollary of our results, we obtain a classification and description of all degenerate irreducible modules over the Hamiltonian Lie-Cartan algebra $P_{2 N}$, along with the description of the singular vectors. This result when $c=0$ was obtained long ago in [10]. 


\section{Preliminaries on Lie pseudoalgebras}

In this section, we review some facts and notation that will be used throughout the paper. For a more detailed treatment, we refer to our previous works [1-3].

\subsection{Bases and filtrations of $H$ and $H^{*}$}

We will denote by $H$ the universal enveloping algebra $U(\mathfrak{d})$ of the Lie algebra $\mathfrak{d}$. Then $H$ is a Hopf algebra with a coproduct $\Delta$, antipode $S$, and counit $\varepsilon$ given by:

$$
\Delta(\partial)=\partial \otimes 1+1 \otimes \partial, \quad S(\partial)=-\partial, \quad \varepsilon(\partial)=0, \quad \partial \in \mathfrak{d} .
$$

We will use the following notation (cf. [12]):

$$
\begin{aligned}
\Delta(h) & =h_{(1)} \otimes h_{(2)}=h_{(2)} \otimes h_{(1)}, \\
(\Delta \otimes \mathrm{id}) \Delta(h) & =(\mathrm{id} \otimes \Delta) \Delta(h)=h_{(1)} \otimes h_{(2)} \otimes h_{(3)}, \\
(S \otimes \mathrm{id}) \Delta(h) & =h_{(-1)} \otimes h_{(2)}, \quad h \in H .
\end{aligned}
$$

Then the axioms of antipode and counit can be written as follows:

$$
\begin{gathered}
h_{(-1)} h_{(2)}=h_{(1)} h_{(-2)}=\varepsilon(h), \\
\varepsilon\left(h_{(1)}\right) h_{(2)}=h_{(1)} \varepsilon\left(h_{(2)}\right)=h,
\end{gathered}
$$

while the fact that $\Delta$ is a homomorphism of algebras translates as:

$$
(f g)_{(1)} \otimes(f g)_{(2)}=f_{(1)} g_{(1)} \otimes f_{(2)} g_{(2)}, \quad f, g \in H
$$

Eqs. (2.5), (2.6) imply the following useful relations:

$$
h_{(-1)} h_{(2)} \otimes h_{(3)}=1 \otimes h=h_{(1)} h_{(-2)} \otimes h_{(3)} .
$$

We are interested in the cocommutative Hopf algebra $H=U(\mathfrak{d})$ serving as coefficient ring for a primitive pseudoalgebra of type $H$, see Section 3 below. As a consequence, we shall henceforth assume that the Lie algebra $\mathfrak{d}$ is even-dimensional.

It will be convenient to work with a basis $\left\{\partial_{1}, \ldots, \partial_{2 N}\right\}$ of $\mathfrak{d}$ and the dual basis $\left\{x^{1}, \ldots, x^{2 N}\right\}$ of $\mathfrak{d}^{*}$. Denote by $c_{i j}^{k}$ the structure constants of $\mathfrak{d}$, so that

$$
\left[\partial_{i}, \partial_{j}\right]=\sum_{k=1}^{2 N} c_{i j}^{k} \partial_{k}, \quad i, j=1, \ldots, 2 N
$$

Note that $H$ has a basis 


$$
\partial^{(I)}=\partial_{1}^{i_{1}} \cdots \partial_{2 N}^{i_{2 N}} / i_{1} ! \cdots i_{2 N} !, \quad I=\left(i_{1}, \ldots, i_{2 N}\right) \in \mathbb{Z}_{+}^{2 N}
$$

The canonical increasing filtration of $H=U(\mathfrak{d})$ is given by

$$
\mathrm{F}^{n} H=\operatorname{span}_{\mathbf{k}}\left\{\partial^{(I)}|| I \mid \leq n\right\}, \quad \text { where } \quad|I|=i_{1}+\cdots+i_{2 N} .
$$

This filtration does not depend on the choice of basis of $\mathfrak{d}$, and is compatible with the Hopf algebra structure of $H$ (see, e.g., [1, Section 2.2] for more details). We have: $\mathrm{F}^{-1} H=\{0\}, \mathrm{F}^{0} H=\mathbf{k}$, and $\mathrm{F}^{1} H=\mathbf{k} \oplus \mathfrak{d}$.

The dual $X=H^{*}:=\operatorname{Hom}_{\mathbf{k}}(H, \mathbf{k})$ is a commutative associative algebra. We will identify $\mathfrak{d}^{*}$ as a subspace of $X$ by letting $\left\langle x^{i}, \partial_{i}\right\rangle=1$ and $\left\langle x^{i}, \partial^{(I)}\right\rangle=0$ for all other basis vectors (2.10). This gives rise to an isomorphism from $X$ to the algebra $\mathcal{O}_{2 N}=$ $\mathbf{k}\left[\left[t^{1}, \ldots, t^{2 N}\right]\right]$ of formal power series in $2 N$ indeterminates, which sends $x^{i}$ to $t^{i}$. The Lie algebra $\mathfrak{d}$ has left and right actions on $X$ by derivations, given by

$$
\begin{aligned}
& \langle\partial x, h\rangle=-\langle x, \partial h\rangle, \\
& \langle x \partial, h\rangle=-\langle x, h \partial\rangle, \quad \partial \in \mathfrak{d}, x \in X, h \in H,
\end{aligned}
$$

where $\partial h$ and $h \partial$ are the products in $H$. These two actions coincide only when $\mathfrak{d}$ is abelian. The difference $\partial x-x \partial$ gives the coadjoint action of $\partial \in \mathfrak{d}$ on $x \in X$.

Throughout the paper, we will be given a trace-form $\chi \in \mathfrak{d}^{*}$, so that $\chi\left(\left[\partial, \partial^{\prime}\right]\right)=0$ for all $\partial, \partial^{\prime} \in \mathfrak{d}$. Then the assignment

$$
\partial \mapsto \bar{\partial}=\partial-\chi(\partial), \quad \partial \in \mathfrak{d}
$$

extends to an associative algebra automorphism $h \mapsto \bar{h}$ of $H$. Note that its inverse is given in the same way but with $-\chi$ in place of $\chi$.

It is convenient to define another basis of $H$ by applying the bar automorphism, thus obtaining elements $\bar{\partial}^{(I)}$. It is easily verified that the corresponding bar filtration on $H$ coincides with $\left\{\mathrm{F}^{n} H\right\}$. The following results will be useful in the rest of the paper.

Lemma 2.1. For any trace-form $\chi \in \mathfrak{d}^{*}$ and $\partial \in \mathfrak{d}$, we have $\partial \chi=\chi \partial=-\chi(\partial)$. In particular,

$$
\begin{aligned}
& \left(x e^{-\chi}\right) \bar{\partial}=(x \partial) e^{-\chi}, \\
& \bar{\partial}\left(x e^{-\chi}\right)=(\partial x) e^{-\chi}, \quad x \in X, \partial \in \mathfrak{d} .
\end{aligned}
$$

Proof. The first claim follows from (2.12) and (2.13). Then (2.15) and (2.16) are derived using that the right and left actions of $\partial$ are derivations of $X$.

Lemma 2.2. (i) The map $h \mapsto S(\overline{S(h)})$ is the inverse to the automorphism $h \mapsto \bar{h}$ of $H$.

(ii) The basis $\left\{x_{I} e^{-\chi}\right\}$ of $X$ is dual to the basis $\left\{S\left(\overline{S\left(\partial^{(I)}\right)}\right)\right\}$ of $H$. 
Proof. (i) The map $h \mapsto S(\overline{S(h)})$ is indeed an automorphism, because $h \mapsto \bar{h}$ is an automorphism and $S$ is an anti-automorphism. It is easy to see that for $\partial \in \mathfrak{d}$, we have $S(\overline{S(\partial)})=\partial+\chi(\partial)$, which is the inverse to the map (2.14).

To prove (ii), first observe that $\langle x, 1\rangle=\left\langle x e^{-\chi}, 1\right\rangle$ for $x \in X$, since $\langle x, 1\rangle$ is the constant term of $x$. Then using (2.12) and (2.16), we find

$$
\begin{aligned}
\delta_{I}^{J} & =\left\langle x_{I}, \partial^{(J)}\right\rangle=\left\langle S\left(\partial^{(J)}\right) x_{I}, 1\right\rangle=\left\langle\left(S\left(\partial^{(J)}\right) x_{I}\right) e^{-\chi}, 1\right\rangle \\
& =\left\langle\overline{S\left(\partial^{(J)}\right)}\left(x_{I} e^{-\chi}\right), 1\right\rangle=\left\langle x_{I} e^{-\chi}, S\left(\overline{S\left(\partial^{(J)}\right)}\right)\right\rangle
\end{aligned}
$$

as claimed.

We introduce a decreasing filtration of $X$ by letting $\mathrm{F}_{n} X=\left(\mathrm{F}^{n} H\right)^{\perp}$ be the set of elements from $X$ that vanish on $\mathrm{F}^{n} H$. Then $\mathrm{F}_{-1} X=X, X / \mathrm{F}_{0} X \simeq \mathbf{k}$, and $\mathrm{F}_{0} X / \mathrm{F}_{1} X \simeq \mathfrak{d}^{*}$. We define a topology of $X$ by considering $\left\{\mathrm{F}_{n} X\right\}$ as a fundamental system of neighborhoods of 0 . We will always consider $X$ with this topology, while $H$ and $\mathfrak{d}$ are endowed with the discrete topology. Then $X$ is linearly compact (see [1, Chapter 6$]$ ), and both the multiplication in $X$ and the left and right actions of $\mathfrak{d}$ on it are continuous.

\subsection{Lie pseudoalgebras and their modules}

Recall from [1, Chapter 3] that a pseudobracket on a left $H$-module $L$ is an $H$-bilinear map

$$
L \otimes L \rightarrow(H \otimes H) \otimes_{H} L, \quad a \otimes b \mapsto[a * b]
$$

where we use the comultiplication $\Delta: H \rightarrow H \otimes H$ to define $(H \otimes H) \otimes_{H} L$. We extend the pseudobracket (2.17) to maps $\left(H^{\otimes 2} \otimes_{H} L\right) \otimes L \rightarrow H^{\otimes 3} \otimes_{H} L$ and $L \otimes\left(H^{\otimes 2} \otimes_{H} L\right) \rightarrow$ $H^{\otimes 3} \otimes_{H} L$ by letting:

$$
\begin{aligned}
& {\left[\left(h \otimes_{H} a\right) * b\right]=\sum_{i}(h \otimes 1)(\Delta \otimes \mathrm{id})\left(g_{i}\right) \otimes_{H} c_{i},} \\
& {\left[a *\left(h \otimes_{H} b\right)\right]=\sum_{i}(1 \otimes h)(\mathrm{id} \otimes \Delta)\left(g_{i}\right) \otimes_{H} c_{i},}
\end{aligned}
$$

where $h \in H^{\otimes 2}, a, b \in L$, and

$$
[a * b]=\sum_{i} g_{i} \otimes_{H} c_{i} \quad \text { with } \quad g_{i} \in H^{\otimes 2}, c_{i} \in L
$$

A Lie pseudoalgebra is a left $H$-module equipped with a pseudobracket satisfying the following skew-commutativity and Jacobi identity axioms: 


$$
\begin{aligned}
{[b * a] } & =-\left(\sigma \otimes_{H} \mathrm{id}\right)[a * b] \\
{[[a * b] * c] } & =[a *[b * c]]-\left((\sigma \otimes \mathrm{id}) \otimes_{H} \mathrm{id}\right)[b *[a * c]] .
\end{aligned}
$$

Here, $\sigma: H \otimes H \rightarrow H \otimes H$ is the permutation of factors, and the compositions $[[a * b] * c]$, $[a *[b * c]]$ are defined using (2.18), (2.19).

Example 2.1. For a Lie algebra $\mathfrak{g}$, the current Lie pseudoalgebra Cur $\mathfrak{g}=H \otimes \mathfrak{g}$ has an action of $H$ by left multiplication on the first tensor factor and a pseudobracket

$$
[(f \otimes a) *(g \otimes b)]=(f \otimes g) \otimes_{H}(1 \otimes[a, b])
$$

for $f, g \in H$ and $a, b \in \mathfrak{g}$.

A module over a Lie pseudoalgebra $L$ is a left $H$-module $V$ together with an $H$-bilinear map

$$
L \otimes V \rightarrow(H \otimes H) \otimes_{H} V, \quad a \otimes v \mapsto a * v
$$

that satisfies $(a, b \in L, v \in V)$ :

$$
[a * b] * v=a *(b * v)-\left((\sigma \otimes \mathrm{id}) \otimes_{H} \mathrm{id}\right)(b *(a * v)) .
$$

An $L$-module $V$ will be called finite if it is finitely generated as an $H$-module, and is called trivial if $a * v=0$ for all $a \in L, v \in V$, i.e., when the action of $L$ on $V$ is trivial. The zero $L$-module is the set $\{0\}$.

Example 2.2. For any module $V_{0}$ over a Lie algebra $\mathfrak{g}$, we have the Cur $\mathfrak{g}$-module $V=$ $H \otimes V_{0}$, with the action given by

$$
(g \otimes b) *(h \otimes v)=(g \otimes h) \otimes_{H}(1 \otimes b v)
$$

for $g, h \in H, b \in \mathfrak{g}$ and $v \in V_{0}$.

Let $U$ and $V$ be two $L$-modules. A map $\beta: U \rightarrow V$ is a homomorphism of $L$-modules if $\beta$ is $H$-linear and satisfies

$$
\left((\mathrm{id} \otimes \mathrm{id}) \otimes_{H} \beta\right)(a * u)=a * \beta(u), \quad a \in L, u \in U
$$

A subspace $W \subset V$ is an $L$-submodule if it is an $H$-submodule and $L * W \subset(H \otimes H) \otimes_{H} W$, where $L * W$ is the linear span of all elements $a * w$ with $a \in L$ and $w \in W$. A submodule $W \subset V$ is called proper if $W \neq V$. An $L$-module $V$ is irreducible (or simple) if it does not contain any non-zero proper $L$-submodules and $L * V \neq\{0\}$. 
Remark 2.1. (i) Let $V$ be a module over a Lie pseudoalgebra $L$ and $W$ be an $H$-submodule of $V$. By [3, Lemma 2.3], for each $a \in L, v \in V$, we can write

$$
a * v=\sum_{I \in \mathbb{Z}_{+}^{2 N}}\left(\partial^{(I)} \otimes 1\right) \otimes_{H} v_{I}^{\prime}, \quad v_{I}^{\prime} \in V,
$$

where the elements $v_{I}^{\prime}$ are uniquely determined by $a$ and $v$. Then $W \subset V$ is an $L$ submodule iff it has the property that all $v_{I}^{\prime} \in W$ whenever $v \in W$.

(ii) Similarly, for each $a \in L, v \in V$, we can write uniquely

$$
a * v=\sum_{I \in \mathbb{Z}_{+}^{2 N}}\left(1 \otimes \partial^{(I)}\right) \otimes_{H} v_{I}^{\prime \prime}, \quad v_{I}^{\prime \prime} \in V
$$

and $W$ is an $L$-submodule iff $v_{I}^{\prime \prime} \in W$ whenever $v \in W$.

\subsection{Twisting of representations}

Let $V$ and $W$ be left $H$-modules. In [1, Chapter 10], we introduced the space $\operatorname{Chom}(V, W)$ of conformal linear maps and showed that when $V$ is finite, one may set up a Lie pseudoalgebra structure on $\operatorname{gc} V:=\operatorname{Chom}(V, V)$ so that defining an action of a Lie pseudoalgebra $L$ on $V$ is equivalent to providing a Lie pseudoalgebra homomorphism $L \rightarrow \operatorname{gc} V$. In addition, if $V$ and $W$ are finite $L$-modules, then $\operatorname{Chom}(V, W)$ has a structure of an $L$-module, given by [1, Remark 10.2].

Definition 2.1 ([2, Definition 4.2]). Let $L$ be a pseudoalgebra over $H=U(\mathfrak{d})$ and $\mathbf{k}$ denote the trivial $H$-module.

(i) For any finite $L$-module $V$, the $L$-module $D(V)=\operatorname{Chom}(V, \mathbf{k})$ is called the dual of $V$. Then $D$ extends to a contravariant functor from the category of finite $L$-modules to itself.

(ii) For any finite $L$-module $V$ and finite-dimensional $\mathfrak{d}$-module $\Pi$, the $L$-module $T_{\Pi}(V)=\operatorname{Chom}(D(V), \Pi)$ is called the twisting of $V$ by $\Pi$. Then $T_{\Pi}$ extends to a covariant functor from the category of finite $L$-modules to itself.

We spell out the meaning of $T_{\Pi}$ in the case where all the $L$-modules are free as $H$ modules, which will be sufficient for our purposes. For a finite $L$-module $V=H \otimes V_{0}$, which is free over $H$, we choose a $\mathbf{k}$-basis $\left\{v_{i}\right\}$ for $V_{0}$, and write the action of $L$ on $V$ in the form

$$
a *\left(1 \otimes v_{i}\right)=\sum_{j}\left(f_{i j} \otimes g_{i j}\right) \otimes_{H}\left(1 \otimes v_{j}\right)
$$

where $a \in L, f_{i j}, g_{i j} \in H$. Then the twisting of $V$ by $\Pi$ is the $L$-module $T_{\Pi}(V)=$ $H \otimes\left(\Pi \otimes V_{0}\right)$, where $H$ acts by a left multiplication on the first factor and 


$$
a *\left(1 \otimes u \otimes v_{i}\right)=\sum_{j}\left(f_{i j} \otimes g_{i j_{(1)}}\right) \otimes_{H}\left(1 \otimes g_{i j(-2)} u \otimes v_{j}\right)
$$

for $a \in L, u \in \Pi$. By [2, Proposition 4.2], $T_{\Pi}(V)$ is an $L$-module and the action of $L$ on it is independent of the choice of basis for $V_{0}$.

Given another $L$-module $V^{\prime}=H \otimes V_{0}^{\prime}$ and a homomorphism $\beta: V \rightarrow V^{\prime}$, we can write

$$
\beta\left(1 \otimes v_{i}\right)=\sum_{j} h_{i j} \otimes v_{j}^{\prime}, \quad h_{i j} \in H
$$

where $\left\{v_{j}^{\prime}\right\}$ is a fixed $\mathbf{k}$-basis for $V_{0}^{\prime}$. Then we have a homomorphism of $L$-modules $T_{\Pi}(\beta): T_{\Pi}(V) \rightarrow T_{\Pi}\left(V^{\prime}\right)$, defined by

$$
T_{\Pi}(\beta)\left(1 \otimes u \otimes v_{i}\right)=\sum_{j} h_{i j(1)} \otimes h_{i j_{(-2)}} u \otimes v_{j}^{\prime}
$$

Moreover, $T_{\Pi}(\beta)$ is independent of the choice of bases [2, Proposition 4.2].

It is now useful to recall two properties showed in [3, Proposition 3.1]. First, the functor $T_{\Pi}$ is exact on free $H$-modules, i.e., if $V \stackrel{\beta}{\rightarrow} V^{\prime} \stackrel{\beta^{\prime}}{\rightarrow} V^{\prime \prime}$ is a short exact sequence of finite free $H$-modules, then the sequence $T_{\Pi}(V) \stackrel{T_{\Pi}(\beta)}{\longrightarrow} T_{\Pi}\left(V^{\prime}\right) \stackrel{T_{\Pi}\left(\beta^{\prime}\right)}{\longrightarrow} T_{\Pi}\left(V^{\prime \prime}\right)$ is exact. Second, the image of $T_{\Pi}(\beta)$ has a finite codimension in $T_{\Pi}\left(V^{\prime}\right)$, whenever the image of $\beta: V \rightarrow V^{\prime}$ has a finite codimension.

\subsection{Annihilation algebras of Lie pseudoalgebras}

For a Lie pseudoalgebra $L$, we let $\mathcal{L}=\mathcal{A}(L)=X \otimes_{H} L$, where as before $X=H^{*}$. We define a Lie bracket on $\mathcal{L}$ by the formula (cf. [1, Eq. (7.2)]):

$$
\left[x \otimes_{H} a, y \otimes_{H} b\right]=\sum_{i}\left(x f_{i}\right)\left(y g_{i}\right) \otimes_{H} c_{i}, \quad \text { if } \quad[a * b]=\sum_{i}\left(f_{i} \otimes g_{i}\right) \otimes_{H} c_{i}
$$

Then $\mathcal{L}$ is a Lie algebra, called the annihilation algebra of $L$ (see [1, Section 7.1]). There is an obvious left action of $H$ on $\mathcal{L}$ given by

$$
h\left(x \otimes_{H} a\right)=h x \otimes_{H} a, \quad h \in H, x \in X, a \in L ;
$$

in particular, the Lie algebra $\mathfrak{d}$ acts on $\mathcal{L}$ by derivations. The semidirect sum $\widetilde{\mathcal{L}}=\mathfrak{d} \ltimes \mathcal{L}$ is called the extended annihilation algebra.

When $L$ is finite, we can define a filtration on $\mathcal{L}$ as follows (see [1, Section 7.4] for more details). We fix a finite-dimensional vector subspace $L_{0}$ of $L$ such that $L=H L_{0}$, and set

$$
\mathrm{F}_{n} \mathcal{L}=\left\{x \otimes_{H} a \in \mathcal{L} \mid x \in \mathrm{F}_{n} X, a \in L_{0}\right\}, \quad n \geq-1 .
$$


The subspaces $\mathrm{F}_{n} \mathcal{L}$ constitute a decreasing filtration of $\mathcal{L}$, satisfying

$$
\left[\mathrm{F}_{m} \mathcal{L}, \mathrm{F}_{n} \mathcal{L}\right] \subset \mathrm{F}_{m+n-\ell} \mathcal{L}, \quad \mathfrak{d}\left(\mathrm{F}_{n} \mathcal{L}\right) \subset \mathrm{F}_{n-1} \mathcal{L}
$$

where $\ell$ is an integer depending only on the choice of $L_{0}$; see [1, Proposition 7.4]. Notice that this filtration of $\mathcal{L}$ depends on the choice of $L_{0}$, but the induced topology does not [1, Lemma 7.2]. With this topology, $\mathcal{L}$ becomes a linearly-compact Lie algebra (see [1]). We set $\mathcal{L}_{n}=\mathrm{F}_{n+\ell} \mathcal{L}$, so that $\left[\mathcal{L}_{m}, \mathcal{L}_{n}\right] \subset \mathcal{L}_{m+n}$. In particular, $\mathcal{L}_{0}$ is a Lie algebra.

We also define a decreasing filtration of $\widetilde{\mathcal{L}}$ by letting $\mathrm{F}_{-1} \widetilde{\mathcal{L}}=\widetilde{\mathcal{L}}, \mathrm{F}_{n} \widetilde{\mathcal{L}}=\mathrm{F}_{n} \mathcal{L}$ for $n \geq 0$, and we set $\widetilde{\mathcal{L}}_{n}=\mathrm{F}_{n+\ell} \widetilde{\mathcal{L}}$. An $\widetilde{\mathcal{L}}$-module $V$ is called conformal if every $v \in V$ is annihilated by some $\mathcal{L}_{n}$; in other words, if $V$ is a topological $\widetilde{\mathcal{L}}$-module when endowed with the discrete topology. The next two results from [1] play a crucial role in our study of representations (see [1], Propositions 9.1 and 14.2, and Lemma 14.4).

Proposition 2.1. Any module $V$ over the Lie pseudoalgebra L has a natural structure of a conformal $\widetilde{\mathcal{L}}$-module, given by the action of $\mathfrak{d}$ on $V$ and by

$$
\left(x \otimes_{H} a\right) \cdot v=\sum_{i}\left\langle x, S\left(f_{i} g_{i(-1)}\right)\right\rangle g_{i(2)} v_{i}, \quad \text { if } \quad a * v=\sum_{i}\left(f_{i} \otimes g_{i}\right) \otimes_{H} v_{i}
$$

for $a \in L, x \in X, v \in V$. Conversely, any conformal $\widetilde{\mathcal{L}}$-module $V$ has a natural structure of an L-module, given by

$$
a * v=\sum_{I \in \mathbb{Z}_{+}^{N}}\left(S\left(\partial^{(I)}\right) \otimes 1\right) \otimes_{H}\left(\left(x_{I} \otimes_{H} a\right) \cdot v\right) .
$$

Moreover, $V$ is irreducible as an L-module iff it is irreducible as an $\widetilde{\mathcal{L}}$-module.

Remark 2.2. In the proof of (2.37), one only uses that $\left\{\partial^{(I)}\right\}$ and $\left\{x_{I}\right\}$ are dual bases of $H$ and $H^{*}$. Then using Lemma 2.2(ii) also gives

$$
a * v=\sum_{I \in \mathbb{Z}_{+}^{N}}\left(\overline{S\left(\partial^{(I)}\right)} \otimes 1\right) \otimes_{H}\left(\left(x_{I} e^{-\chi} \otimes_{H} a\right) \cdot v\right)
$$

Lemma 2.3. Let $L$ be a finite Lie pseudoalgebra and $V$ be a finite L-module. For $n \geq$ $-1-\ell$, let

$$
\operatorname{ker}_{n} V=\left\{v \in V \mid \mathcal{L}_{n} v=0\right\}
$$

so that, for example, $\operatorname{ker}_{-1-\ell} V=\operatorname{ker} V$ and $V=\bigcup \operatorname{ker}_{n} V$. Then all vector spaces $\operatorname{ker}_{n} V / \operatorname{ker} V$ are finite dimensional. In particular, if $\operatorname{ker} V=\{0\}$, then every vector $v \in V$ is contained in a finite-dimensional subspace invariant under $\mathcal{L}_{0}$. 


\section{5. $W(\mathfrak{d})$ and its annihilation algebra}

One of the most important Lie pseudoalgebras is $W(\mathfrak{d})=H \otimes \mathfrak{d}$, with the Lie pseudobracket (see [1, Section 8.1]):

$$
\begin{aligned}
{[(f \otimes a) *(g \otimes b)] } & =(f \otimes g) \otimes_{H}(1 \otimes[a, b]) \\
& -(f \otimes g a) \otimes_{H}(1 \otimes b)+(f b \otimes g) \otimes_{H}(1 \otimes a),
\end{aligned}
$$

for $f, g \in H, a, b \in \mathfrak{d}$. The formula

$$
(f \otimes a) * h=-(f \otimes h a) \otimes_{H} 1
$$

defines the structure of a $W(\mathfrak{d})$-module on $H$.

We denote the annihilation algebra of $W(\mathfrak{d})$ by

$$
\mathcal{W}=\mathcal{A}(W(\mathfrak{d}))=X \otimes_{H}(H \otimes \mathfrak{d}) \simeq X \otimes \mathfrak{d}
$$

The Lie bracket in $\mathcal{W}$ is given by $(x, y \in X, a, b \in \mathfrak{d})$ :

$$
[x \otimes a, y \otimes b]=x y \otimes[a, b]-x(y a) \otimes b+(x b) y \otimes a .
$$

The extended annihilation algebra of $W(\mathfrak{d})$ is $\widetilde{\mathcal{W}}=\mathfrak{d} \ltimes \mathcal{W}$, where

$$
[\partial, x \otimes a]=\partial x \otimes a, \quad \partial, a \in \mathfrak{d}, x \in X
$$

The Lie algebra $\mathcal{W}$ has a decreasing filtration

$$
\mathcal{W}_{n}=\mathrm{F}_{n} \mathcal{W}=\mathrm{F}_{n} X \otimes \mathfrak{d}, \quad n \geq-1
$$

satisfying $\mathcal{W}_{-1}=\mathcal{W}$ and $\left[\mathcal{W}_{i}, \mathcal{W}_{j}\right] \subset \mathcal{W}_{i+j}$. Note that $\mathcal{W} / \mathcal{W}_{0} \simeq \mathbf{k} \otimes \mathfrak{d} \simeq \mathfrak{d}$ and $\mathcal{W}_{0} / \mathcal{W}_{1} \simeq$ $\mathfrak{d}^{*} \otimes \mathfrak{d}$.

Lemma 2.4 ([2]). The map from $\mathcal{W}_{0} / \mathcal{W}_{1}$ to $\mathfrak{d} \otimes \mathfrak{d}^{*} \simeq \mathfrak{g l} \mathfrak{d}$, defined by $x \otimes a \bmod \mathcal{W}_{1} \mapsto-a \otimes\left(x \bmod \mathrm{F}_{1} X\right), \quad x \in \mathrm{F}_{0} X, a \in \mathfrak{d}$,

is a Lie algebra isomorphism. Under this isomorphism, the adjoint action of $\mathcal{W}_{0} / \mathcal{W}_{1}$ on $\mathcal{W} / \mathcal{W}_{0}$ coincides with the standard action of $\mathfrak{g l} \mathfrak{d}$ on $\mathfrak{d}$.

The action (2.40) of $W(\mathfrak{d})$ on $H$ induces a corresponding action of the annihilation algebra $\mathcal{W}$ on $X$ given by

$$
(x \otimes a) y=-x(y a), \quad x, y \in X, a \in \mathfrak{d} .
$$


Since $\mathfrak{d}$ acts on $X$ by continuous derivations, the Lie algebra $\mathcal{W}$ acts on $X$ by continuous derivations. The isomorphism $X \simeq \mathcal{O}_{2 N}$ induces a Lie algebra homomorphism $\varphi$ from $\mathcal{W}$ to $W_{2 N}=\operatorname{Der} \mathcal{O}_{2 N}$, the Lie algebra of continuous derivations of the algebra $\mathcal{O}_{2 N}=$ $\mathbf{k}\left[\left[t^{1}, \ldots, t^{2 N}\right]\right]$. In fact, $\varphi$ is an isomorphism compatible with the filtrations (see [2, Proposition 3.1]). We recall that the canonical filtration of the Lie-Cartan algebra $W_{2 N}$ is given explicitly by

$$
\mathrm{F}_{p} W_{2 N}=\left\{\sum_{i=1}^{2 N} f_{i} \frac{\partial}{\partial t^{i}} \mid f_{i} \in \mathrm{F}_{p} \mathcal{O}_{2 N}\right\}, \quad p \geq-1
$$

where $\mathrm{F}_{p} \mathcal{O}_{2 N}$ is the $(p+1)$-st power of the maximal ideal $\left(t^{1}, \ldots, t^{2 N}\right)$ of $\mathcal{O}_{2 N}$.

It is well known that all continuous derivations of the Lie algebra $W_{2 N}$ are inner (see e.g. [1, Proposition 6.4(i)]). Hence, the same is true for $\mathcal{W}$. Since every non-zero $\partial \in \mathfrak{d}$ acts as a non-zero continuous derivation of $\mathcal{W}$ by (2.43), we obtain an injective Lie algebra homomorphism $\gamma: \mathfrak{d} \hookrightarrow \mathcal{W}$ such that the elements

$$
\widetilde{\partial}:=\partial-\gamma(\partial) \in \widetilde{\mathcal{W}} \quad(\partial \in \mathfrak{d})
$$

centralize $\mathcal{W}$. The set $\widetilde{\mathfrak{d}}$ of all $\widetilde{\partial}$ is a subalgebra of $\widetilde{\mathcal{W}}$, which is isomorphic to $\mathfrak{d}$ under the map $\partial \mapsto \widetilde{\partial}$ (see [2, Proposition 3.2]). Moreover, by [2, Lemma 3.3],

$$
\widetilde{\partial}=\partial+1 \otimes \partial-\operatorname{ad} \partial \bmod \mathcal{W}_{1}, \quad \partial \in \mathfrak{d},
$$

where ad $\partial$ is understood as an element of $\mathfrak{g l} \mathfrak{d} \simeq \mathcal{W}_{0} / \mathcal{W}_{1}$ via Lemma 2.4.

\section{Primitive Lie pseudoalgebras of type $H$}

In this section, we introduce the main objects of our study: the Lie pseudoalgebra $H(\mathfrak{d}, \chi, \omega)$ and its annihilation Lie algebra (see [1, Chapter 8]). We also review the unique embedding of $H(\mathfrak{d}, \chi, \omega)$ into the Lie pseudoalgebra $W(\mathfrak{d})$ and the induced homomorphism of annihilation algebras.

\subsection{Symplectic Lie algebra}

Let $\omega \in \mathfrak{d}^{*} \wedge \mathfrak{d}^{*}$ be a nondegenerate skew-symmetric 2 -form, so that

$$
\underbrace{\omega \wedge \cdots \wedge \omega}_{N} \neq 0, \quad \operatorname{dim} \mathfrak{d}=2 N
$$

We set $\omega_{i j}=\omega\left(\partial_{i} \wedge \partial_{j}\right)$ and denote by $\left(r^{i j}\right)$ the inverse matrix to $\left(\omega_{i j}\right)$ :

$$
\sum_{k=1}^{2 N} r^{i k} \omega_{k j}=\delta_{j}^{i}, \quad i, j=1, \ldots, 2 N .
$$


We identify End $\mathfrak{d}$ with $\mathfrak{d} \otimes \mathfrak{d}^{*}$ so that the elementary matrix $e_{i}^{j} \in$ End $\mathfrak{d}$ is identified with the element $\partial_{i} \otimes x^{j} \in \mathfrak{d} \otimes \mathfrak{d}^{*}$, where $e_{i}^{j}\left(\partial_{k}\right)=\delta_{k}^{j} \partial_{i}$. Notice that $(\partial \otimes x)\left(\partial^{\prime}\right)=\left\langle x, \partial^{\prime}\right\rangle \partial$, and the composition $(\partial \otimes x) \circ\left(\partial^{\prime} \otimes x^{\prime}\right)$ equals $\left\langle x, \partial^{\prime}\right\rangle \partial \otimes x^{\prime}$. We will raise indices using the matrix $\left(r^{i j}\right)$ and lower them using $\left(\omega_{i j}\right)$. In particular,

$$
e^{i j}=\partial^{i} \otimes x^{j}=\sum_{k=1}^{2 N} r^{i k} e_{k}^{j},
$$

where

$$
\partial^{i}=\sum_{k=1}^{2 N} r^{i k} \partial_{k}, \quad \omega\left(\partial^{i} \wedge \partial_{j}\right)=\delta_{j}^{i}
$$

Conversely, we have

$$
\partial_{k}=\sum_{j=1}^{2 N} \omega_{k j} \partial^{j}
$$

Denote by $\mathfrak{s p} \mathfrak{d}=\mathfrak{s p}(\mathfrak{d}, \omega)$ the Lie algebra of all $A \in \mathfrak{g l} \mathfrak{d}$ such that $A \cdot \omega=0$, i.e.,

$$
\omega\left(A \partial_{i} \wedge \partial_{j}\right)+\omega\left(\partial_{i} \wedge A \partial_{j}\right)=0, \quad i, j=1, \ldots, 2 N
$$

The Lie algebra $\mathfrak{s p} \mathfrak{d}$ is isomorphic to $\mathfrak{s p}_{2 N}$ and, in particular, is simple. It is easy to see that the elements

$$
f^{i j}=-\frac{1}{2}\left(e^{i j}+e^{j i}\right)=f^{j i}, \quad 1 \leq i \leq j \leq 2 N
$$

form a basis of $\mathfrak{s p} \mathfrak{d}$. We will denote by $R(\lambda)$ the irreducible $\mathfrak{s p} \mathfrak{d}$-module with highest weight $\lambda$, and by $\pi_{n}$ the fundamental weights of $\mathfrak{s p d}$. For example, $R\left(\pi_{1}\right) \simeq \mathfrak{d}$ is the vector representation. We set $R\left(\pi_{0}\right)=\mathbf{k}$ and $R\left(\pi_{n}\right)=\{0\}$ if $n<0$ or $n>N$.

Example 3.1. Let us choose the basis of $\mathfrak{d}$ to be symplectic, i.e.,

$$
\omega\left(\partial_{i} \wedge \partial_{i+N}\right)=1=-\omega\left(\partial_{i+N} \wedge \partial_{i}\right), \quad \omega\left(\partial_{i} \wedge \partial_{j}\right)=0 \text { for } \quad|i-j| \neq N .
$$

Then we have:

$$
\partial^{i}=-\partial_{i+N}, \quad \partial^{i+N}=\partial_{i}, \quad 1 \leq i \leq N
$$

which implies

$$
e^{i j}=-e_{i+N}^{j}, \quad e^{i+N, j}=e_{i}^{j}, \quad 1 \leq i \leq N, 1 \leq j \leq 2 N
$$




\subsection{Definition of $H(\mathfrak{d}, \chi, \omega)$}

Let again $\chi \in \mathfrak{d}^{*}$ be a trace form and $\omega \in \mathfrak{d}^{*} \wedge \mathfrak{d}^{*}$ be a nondegenerate skew-symmetric 2 -form on $\mathfrak{d}$. From now on we will assume that $\omega$ and $\chi$ satisfy the equation

$$
\left(\omega\left(\left[a_{1}, a_{2}\right] \wedge a_{3}\right)-\chi\left(a_{1}\right) \omega\left(a_{2} \wedge a_{3}\right)\right)+\text { cyclic }=0, \quad a_{1}, a_{2}, a_{3} \in \mathfrak{d},
$$

where "cyclic" here and further means applying the two non-trivial cyclic permutations to the indices $1,2,3$. We fix a basis $\left\{\partial_{1}, \ldots, \partial_{2 N}\right\}$ of $\mathfrak{d}$ as in Section 2.1, and let

$$
r=\sum_{i, j=1}^{2 N} r^{i j} \partial_{i} \otimes \partial_{j}=\sum_{i=1}^{2 N} \partial_{i} \otimes \partial^{i}=-\sum_{i=1}^{2 N} \partial^{i} \otimes \partial_{i}
$$

(cf. (3.4)). Notice that $r$ is skew-symmetric and independent of the choice of basis. We define $s \in \mathfrak{d}$ by the property

$$
\chi(a)=\left(\iota_{s} \omega\right)(a)=\omega(s \wedge a), \quad a \in \mathfrak{d} .
$$

Then $(3.11)$ and $\chi([\mathfrak{d}, \mathfrak{d}])=0$ are equivalent to the following system of equations for $r$ and $s$ (see [1, Lemma 8.5]):

$$
\begin{aligned}
{[r, \Delta(s)] } & =0, \\
\left(\left[r_{12}, r_{13}\right]+r_{12} s_{3}\right)+\text { cyclic } & =0,
\end{aligned}
$$

where we use the standard notation $r_{12}=r \otimes 1, s_{3}=1 \otimes 1 \otimes s$, etc. Consider a free rank-one $H$-module $H e$ with a generator $e$. It follows from [1, Lemma 8.7] that one may endow $\mathrm{He}$ with a unique Lie pseudoalgebra bracket extending

$$
[e * e]=(r+s \otimes 1-1 \otimes s) \otimes_{H} e .
$$

The resulting Lie pseudoalgebra is denoted $H(\mathfrak{d}, \chi, \omega)$. There is an injective homomorphism of Lie pseudoalgebras

$$
\iota: H(\mathfrak{d}, \chi, \omega) \rightarrow W(\mathfrak{d}), \quad e \mapsto-r+1 \otimes s
$$

where $W(\mathfrak{d})=H \otimes \mathfrak{d}$ is from Section 2.5 (see [1, Lemma 8.3]). Moreover, this is the unique non-trivial homomorphism from $H(\mathfrak{d}, \chi, \omega)$ to $W(\mathfrak{d})$, by [1, Theorem 13.7]. From now on, we will often identify $H(\mathfrak{d}, \chi, \omega)$ with its image in $W(\mathfrak{d})$.

Note that, by (3.4) and (3.13), we have

$$
s=\sum_{i=1}^{2 N} \chi\left(\partial_{i}\right) \partial^{i}=-\sum_{i=1}^{2 N} \chi\left(\partial^{i}\right) \partial_{i} .
$$


This allows us to rewrite (3.16) in the form

$$
[e * e]=\sum_{i, j=1}^{2 N} r^{i j}\left(\bar{\partial}_{i} \otimes \bar{\partial}_{j}\right) \otimes_{H} e=\sum_{i=1}^{2 N}\left(\bar{\partial}_{i} \otimes \bar{\partial}^{i}\right) \otimes_{H} e,
$$

where $\bar{\partial}$ is given by (2.14). Thus,

$$
\iota(e)=-r+1 \otimes s=-\sum_{i=1}^{2 N} \bar{\partial}_{i} \otimes \partial^{i}=\sum_{i=1}^{2 N} \bar{\partial}^{i} \otimes \partial_{i} .
$$

Observe also that $\chi(s)=\omega(s \wedge s)=0$ and so $\bar{s}=s$.

Applying the multiplication map of $U(\mathfrak{d})$ to (3.12) shows that

$$
\sum_{i=1}^{2 N} \partial_{i} \partial^{i}=-\sum_{i=1}^{2 N} \partial^{i} \partial_{i}
$$

so that the element

$$
U(\mathfrak{d}) \ni \rho:=\sum_{i=1}^{2 N} \partial_{i} \partial^{i}=\frac{1}{2} \sum_{i=1}^{2 N}\left[\partial_{i}, \partial^{i}\right]=\frac{1}{2} \sum_{i, j=1}^{2 N} r^{i j}\left[\partial_{i}, \partial_{j}\right]
$$

lies in $\mathfrak{d} \subset U(\mathfrak{d})$. We consider the linear function

$$
\phi=-\chi+\iota_{\rho} \omega=\iota_{\rho-s} \omega \in \mathfrak{d}^{*}
$$

Here $\iota_{\rho} \omega \in \mathfrak{d}^{*}$ is defined by $\left(\iota_{\rho} \omega\right)(a)=\omega(\rho \wedge a)$ for $a \in \mathfrak{d}$, and we used that $\chi=\iota_{s} \omega$.

Remark 3.1. By [1, Remark 8.5], the above embedding (3.17) realizes $H(\mathfrak{d}, \chi, \omega)$ as a subalgebra of the Lie pseudoalgebra $S(\mathfrak{d}, \phi) \subset W(\mathfrak{d})$. Note that for $N=1$, we have $H(\mathfrak{d}, \chi, \omega)=S(\mathfrak{d}, \phi)=S(\mathfrak{d},-\chi+\operatorname{tr}$ ad $) ;$ see $[1$, Example 8.1].

As $S(\mathfrak{d}, \phi)$ is a well-defined subalgebra of $W(\mathfrak{d})$, we know that $\phi$ must be a trace form on $\mathfrak{d}$. This becomes more evident from another expression for it, which will be useful in the sequel.

Lemma 3.1. With the above notation (3.21), (3.22), we have $\phi=-N \chi+\operatorname{tr}$ ad. In particular, $\phi$ is a trace form on $\mathfrak{d}$.

Proof. First observe that, by (3.4), for any linear operator $A$ on $\mathfrak{d}$, we have

$$
\operatorname{tr} A=\sum_{i=1}^{2 N} \omega\left(A \partial^{i} \wedge \partial_{i}\right)=-\sum_{i=1}^{2 N} \omega\left(A \partial_{i} \wedge \partial^{i}\right)
$$


Then, using (3.4), (3.11), (3.13) and (3.18), we find for $a \in \mathfrak{d}$ :

$$
\begin{aligned}
2 \omega(\rho \wedge a)= & \sum_{i=1}^{2 N} \omega\left(\left[\partial_{i}, \partial^{i}\right] \wedge a\right) \\
= & -\sum_{i=1}^{2 N}\left(\omega\left(\left[\partial^{i}, a\right] \wedge \partial_{i}\right)+\omega\left(\left[a, \partial_{i}\right] \wedge \partial^{i}\right)\right) \\
& +\sum_{i=1}^{2 N}\left(\chi\left(\partial_{i}\right) \omega\left(\partial^{i} \wedge a\right)+\chi\left(\partial^{i}\right) \omega\left(a \wedge \partial_{i}\right)+\chi(a) \omega\left(\partial_{i} \wedge \partial^{i}\right)\right) \\
= & 2 \operatorname{tr} \operatorname{ad} a+2 \omega(s \wedge a)-2 N \chi(a) \\
= & 2 \operatorname{tr} \operatorname{ad} a+(2-2 N) \chi(a) .
\end{aligned}
$$

Therefore, $\iota_{\rho} \omega=(1-N) \chi+\operatorname{tr}$ ad, as claimed.

\subsection{Annihilation algebra of $H(\mathfrak{d}, \chi, \omega)$}

We will denote by $\mathcal{P}$ the annihilation algebra of the Lie pseudoalgebra $H(\mathfrak{d}, \chi, \omega)$ (see Section 2.4 and [1, Section 7.1]). By definition, we have

$$
\mathcal{P}=\mathcal{A}(H(\mathfrak{d}, \chi, \omega))=X \otimes_{H} H(\mathfrak{d}, \chi, \omega)=X \otimes_{H} H e,
$$

which sometimes will be identified with $X$ via the map $x \otimes_{H} h e \mapsto x h$. The Lie bracket on $\mathcal{P}$ is given by (cf. (2.32), (3.19)):

$$
[x, y]=\sum_{i, j=1}^{2 N} r^{i j}\left(x \bar{\partial}_{i}\right)\left(y \bar{\partial}_{j}\right)=\sum_{i=1}^{2 N}\left(x \bar{\partial}_{i}\right)\left(y \bar{\partial}^{i}\right), \quad x, y \in X .
$$

We define a decreasing filtration on $\mathcal{P}$ by

$$
\mathcal{P}_{n}=\mathrm{F}_{n} \mathcal{P}=\mathrm{F}_{n+1} X \otimes_{H} e \simeq \mathrm{F}_{n+1} X, \quad n \geq-2,
$$

which is obtained as in Section 2.4 by choosing $L_{0}=\mathbf{k} e$. The canonical injection $\iota$ of the subalgebra $H(\mathfrak{d}, \chi, \omega)$ in $W(\mathfrak{d})$ induces a Lie algebra homomorphism $\iota_{*}: \mathcal{P} \rightarrow \mathcal{W}$, which is given explicitly by (cf. (3.20)):

$$
\iota_{*}(x)=-\sum_{i=1}^{2 N} x \bar{\partial}_{i} \otimes \partial^{i}=\sum_{i=1}^{2 N} x \bar{\partial}^{i} \otimes \partial_{i}, \quad x \in X \simeq \mathcal{P} .
$$

We will also denote by $\iota_{*}$ the corresponding Lie algebra homomorphism of extended annihilation algebras $\widetilde{\mathcal{P}} \rightarrow \widetilde{\mathcal{W}}$, defined by $(3.26)$ and by $\iota_{*}(\partial)=\partial$ for $\partial \in \mathfrak{d}$.

The map $\iota_{*}$ is not injective, contrary to what happens with primitive Lie pseudoalgebras of all other types. 
Lemma 3.2. The map $\iota_{*}: \mathcal{P} \rightarrow \mathcal{W}$ has a 1-dimensional kernel, which is spanned over $\mathbf{k}$ by $e^{-\chi} \equiv e^{-\chi} \otimes_{H}$ e and is contained in the center of $\mathcal{P}$.

Proof. Using (3.26) and (2.15), we obtain $\iota_{*}\left(x e^{-\chi}\right)=\sum_{i}\left(x \partial^{i}\right) e^{-\chi} \otimes \partial_{i}$. This is zero if and only if $x \partial^{i}=0$ for all $i$, which only happens when $x$ lies in $\mathbf{k} \subset X$. The fact that $e^{-\chi}$ is central in $\mathcal{P}$ follows immediately from (3.24) and (2.15).

From now on, we will denote by $\mathcal{H}:=\iota_{*}(\mathcal{P})$ the image of $\mathcal{P}$ in $\mathcal{W}$. It has two filtrations, induced by the filtrations of $\mathcal{P}$ and of $\mathcal{W}$, which coincide due to the next lemma.

Lemma 3.3. The filtrations of $\mathcal{P}$ and $\mathcal{W}$ are compatible, i.e.,

$$
\mathcal{H}_{n}:=\iota_{*}\left(\mathcal{P}_{n}\right)=\iota_{*}(\mathcal{P}) \cap \mathcal{W}_{n}, \quad n \geq-2 .
$$

Moreover, $\left[\mathcal{P}_{m}, \mathcal{P}_{n}\right] \subset \mathcal{P}_{m+n}$ for all $m, n \in \mathbb{Z}$.

Proof. This follows from (2.44), (3.24)-(3.26) and the facts that $\left(\mathrm{F}_{n+1} X\right) \mathfrak{d} \subset \mathrm{F}_{n} X$ and $\left(\mathrm{F}_{m} X\right)\left(\mathrm{F}_{n} X\right) \subset \mathrm{F}_{m+n+1} X$.

By Lemma 3.2, there is a central extension of Lie algebras

$$
0 \rightarrow \mathbf{k} e^{-\chi} \rightarrow \mathcal{P} \stackrel{\iota_{*}}{\rightarrow} \mathcal{H} \rightarrow 0
$$

Composing the isomorphism $\varphi: \mathcal{W} \rightarrow W_{2 N}$ with the homomorphism $\iota_{*}: \mathcal{P} \rightarrow \mathcal{W}$, one obtains a Lie algebra homomorphism $\mathcal{P} \rightarrow W_{2 N}$ with kernel $\mathbf{k} e^{-\chi}$. Its image does not necessarily coincide with the Lie-Cartan algebra $H_{2 N} \subset W_{2 N}$ of Hamiltonian vector fields, but it does up to a change of variables, as we will see below.

Let us review the definition of $H_{2 N}$ and its irreducible central extension $P_{2 N}$. We have $P_{2 N}=\mathcal{O}_{2 N}$ with the Lie bracket

$$
[f, g]=\sum_{i=1}^{N} \frac{\partial f}{\partial t^{i}} \frac{\partial g}{\partial t^{N+i}}-\frac{\partial f}{\partial t^{N+i}} \frac{\partial g}{\partial t^{i}}, \quad f, g \in \mathcal{O}_{2 N}
$$

(which is known as the Poisson bracket). There is a homomorphism $P_{2 N} \rightarrow W_{2 N}$ given by

$$
f \mapsto \sum_{i=1}^{N} \frac{\partial f}{\partial t^{i}} \frac{\partial}{\partial t^{N+i}}-\frac{\partial f}{\partial t^{N+i}} \frac{\partial}{\partial t^{i}}
$$

whose image is $H_{2 N}$ and whose kernel is $\mathbf{k}$. Note that $H_{2 N}$ consists of all vector fields from $W_{2 N}$ annihilating the standard symplectic form $\sum_{i=1}^{N} d t^{i} \wedge d t^{N+i}$. 
Proposition 3.1. There exists a ring automorphism $\psi$ of $\mathcal{O}_{2 N}$, which induces a Lie algebra automorphism $\psi$ of $W_{2 N}$, such that the image of $\mathcal{H}$ in $W_{2 N}$ under the isomorphism $\varphi: \mathcal{W} \rightarrow W_{2 N}$ coincides with $\psi\left(H_{2 N}\right)$. Furthermore, $\psi$ is compatible with the filtrations, and it induces the identity map on the associated graded algebras, i.e.,

$$
(\psi-\mathrm{id}) \mathrm{F}_{p} W_{2 N} \subset \mathrm{F}_{p+1} W_{2 N}, \quad p \geq-1 .
$$

Proof. The same as that of [2, Proposition 3.6] and [3, Proposition 4.1].

As a consequence of Proposition 3.1, $\mathcal{H} \simeq H_{2 N}$ and $\mathcal{P} \simeq P_{2 N}$. In the following, we will need certain explicit elements of $\mathcal{H}$.

Lemma 3.4. The Lie algebra homomorphism $\iota_{*}: \mathcal{P} \rightarrow \mathcal{W}$ identifies the following elements:

(i) $e^{-\chi} \otimes_{H} e \mapsto 0$,

(ii) $x^{k} e^{-\chi} \otimes_{H} e \mapsto e^{-\chi} \otimes \partial^{k}-\sum_{1 \leq i<j \leq 2 N} c_{i j}^{k} x^{j} e^{-\chi} \otimes \partial^{i} \bmod \mathcal{W}_{1}$,

(iii) $x^{i} x^{j} e^{-\chi} \otimes_{H} e \mapsto 2 f^{i j} \bmod \mathcal{W}_{1}$.

Proof. The proof is straightforward, using (3.26). Note that $f^{i j} \in \mathfrak{g l} \mathfrak{d}$ are understood via the identification $\mathfrak{g l} \mathfrak{d} \simeq \mathcal{W}_{0} / \mathcal{W}_{1}$ (see Lemma 2.4).

Corollary 3.1. We have a Lie algebra isomorphism $\mathcal{H}_{0} / \mathcal{H}_{1} \simeq \mathfrak{s p} \mathfrak{d}$.

Proof. The composition $\mathcal{H}_{0} \rightarrow \mathcal{W}_{0} \rightarrow \mathcal{W}_{0} / \mathcal{W}_{1}$ is injective on the linear span of elements $x^{i} x^{j} \otimes_{H} e$, which generate a subalgebra isomorphic to $\mathfrak{s p} \mathfrak{d}$.

For future use, we introduce the following linear operators on $\mathfrak{d}(1 \leq k \leq 2 N)$ :

$$
\operatorname{ad}^{\mathfrak{s p}} \partial^{k}:=\operatorname{ad} \partial^{k}+\partial^{k} \otimes \chi+\frac{1}{2} \sum_{i, j=1}^{2 N} c_{i j}^{k} e^{i j}-\frac{1}{2} \chi\left(\partial^{k}\right) I
$$

where $I$ denotes the identity operator and as before we identify $\mathfrak{d} \otimes \mathfrak{d}^{*} \simeq$ End $\mathfrak{d}$. Recall also that $e^{i j}$ are given by (3.3), $f^{i j}$ by (3.7), and $c_{i j}^{k}$ are the structure constants of $\mathfrak{d}$ given by (2.9). The notation $\operatorname{ad}^{\mathfrak{s p}}$ is explained by the next lemma (note that it differs from the notation used in [3]).

Lemma 3.5. For every $k=1, \ldots, 2 N$, we have $\operatorname{ad}^{\mathfrak{s p}} \partial^{k} \in \mathfrak{s p} \mathfrak{d}$. Moreover, $\operatorname{ad}^{\mathfrak{s p}} \partial^{k}$ is the image of ad $\partial^{k}+\partial^{k} \otimes \chi$ under the projection $\pi: \mathfrak{g l} \mathfrak{d} \rightarrow \mathfrak{s p} \mathfrak{d}$ defined by $\pi\left(e^{i j}\right)=-f^{i j}$.

Proof. Denoting the operator (3.30) by $A$, we see that

$$
A \partial_{j}=\left[\partial^{k}, \partial_{j}\right]+\chi\left(\partial_{j}\right) \partial^{k}-\frac{1}{2} \chi\left(\partial^{k}\right) \partial_{j}+\frac{1}{2} \sum_{i=1}^{2 N} c_{i j}^{k} \partial^{i}
$$


Then, using (3.4) and (3.11), we get:

$$
\begin{aligned}
& \omega\left(A \partial_{j} \wedge \partial_{\ell}\right)+\omega\left(\partial_{j} \wedge A \partial_{\ell}\right) \\
& \quad=\omega\left(\left[\partial^{k}, \partial_{j}\right] \wedge \partial_{\ell}\right)+\chi\left(\partial_{j}\right) \omega\left(\partial^{k} \wedge \partial_{\ell}\right)-\frac{1}{2} \chi\left(\partial^{k}\right) \omega\left(\partial_{j} \wedge \partial_{\ell}\right)+\frac{1}{2} c_{\ell j}^{k} \\
& \quad+\omega\left(\partial_{j} \wedge\left[\partial^{k}, \partial_{\ell}\right]\right)+\chi\left(\partial_{\ell}\right) \omega\left(\partial_{j} \wedge \partial^{k}\right)-\frac{1}{2} \chi\left(\partial^{k}\right) \omega\left(\partial_{j} \wedge \partial_{\ell}\right)-\frac{1}{2} c_{j \ell}^{k} \\
& \quad=\omega\left(\left[\partial_{\ell}, \partial_{j}\right] \wedge \partial^{k}\right)+c_{\ell j}^{k} \\
& \quad=\sum_{i=1}^{2 N} c_{\ell j}^{i} \omega\left(\partial_{i} \wedge \partial^{k}\right)+c_{\ell j}^{k} \\
& =0
\end{aligned}
$$

Therefore, $A \in \mathfrak{s p} \mathfrak{d}$. Note that $\pi$ is indeed a projection, since $\pi\left(f^{i j}\right)=f^{i j}$. To finish the proof, we need to show that $\pi\left(A-\operatorname{ad} \partial^{k}-\partial^{k} \otimes \chi\right)=0$. By (3.5), we have

$$
I=\sum_{i=1}^{2 N} \partial_{i} \otimes x^{i}=\sum_{i, j=1}^{2 N} \omega_{i j} e^{j i}
$$

which implies $\pi(I)=0$, because $\pi\left(e^{i j}\right)=\pi\left(e^{j i}\right)$ while $\omega_{i j}=-\omega_{j i}$. For the same reason,

$$
\pi\left(\sum_{i, j=1}^{2 N} c_{i j}^{k} e^{i j}\right)=0
$$

thus completing the proof.

Extending (3.30) by linearity, we obtain a linear map $\operatorname{ad}^{\mathfrak{s p}}: \mathfrak{d} \rightarrow \mathfrak{s p} \mathfrak{d}$, which does not depend on the choice of basis due to Lemma 3.5.

\subsection{The normalizers $\mathcal{N}_{\mathcal{H}}$ and $\mathcal{N}_{\mathcal{P}}$}

Recall the filtration (3.25) of the annihilation algebra $\mathcal{P}$ of the Lie pseudoalgebra $H(\mathfrak{d}, \chi, \omega)$. Our next goal is to compute the normalizer of $\mathcal{P}_{0}$ inside the extended annihilation algebra $\widetilde{\mathcal{P}}$. The first step will be to find the normalizer of $\mathcal{H}_{0}=\iota_{*}\left(\mathcal{P}_{0}\right)$ in $\widetilde{\mathcal{H}}=\mathfrak{d} \ltimes \mathcal{H} \subset \widetilde{\mathcal{W}}$.

It is well known that every continuous derivation of the Lie algebra $H_{2 N}$ is the sum of an inner derivation and a scalar multiple of ad $E$, where

$$
E:=\sum_{i=1}^{2 N} t^{i} \frac{\partial}{\partial t^{i}} \in \mathrm{F}_{0} W_{2 N}
$$


is the Euler vector field (see e.g. [1, Proposition 6.4(i)]). Due to Proposition 3.1, the normalizer of $\mathcal{H}$ in $\mathcal{W}$ coincides with $\mathcal{H}+\mathbf{k} \mathcal{E}$, where

$$
\mathcal{E}=\varphi^{-1} \psi(E) \in \mathcal{W}_{0}
$$

Moreover, $\psi(E)=E \bmod \mathrm{F}_{1} W_{2 N}$, and by [2, (3.16)],

$$
\varphi^{-1}(E)=-\sum_{i=1}^{2 N} x^{i} \otimes \partial_{i} \bmod \mathcal{W}_{1}
$$

Therefore, the image of $\mathcal{E}$ in $\mathcal{W}_{0} / \mathcal{W}_{1} \simeq \mathfrak{g l} \mathfrak{d}$ is the identity operator $I$ (see Lemma 2.4).

Every non-zero $\partial \in \mathfrak{d}$ acts as a non-zero continuous derivation of $\mathcal{H}$; hence, we get an injective Lie algebra homomorphism $\gamma: \mathfrak{d} \hookrightarrow \mathcal{H}+\mathbf{k} \mathcal{E}$ such that $\widetilde{\partial}=\partial-\gamma(\partial) \in \widetilde{\mathcal{W}}$ centralizes $\mathcal{H}$. Since the centralizer of $\mathcal{H}$ in $\mathcal{W}$ is trivial, the elements $\widetilde{\partial}$ coincide with those constructed from the Lie algebra $\mathcal{W}$ (see (2.47)). As before, the map $\partial \mapsto \widetilde{\partial}$ is a Lie algebra isomorphism from $\mathfrak{d}$ onto a subalgebra $\widetilde{\mathfrak{d}}$ of $\widetilde{\mathcal{W}}$. If we add a suitable scalar multiple of $\mathcal{E}$ to $\widetilde{\partial}$, we will obtain a unique $\widehat{\partial} \in \widetilde{\mathcal{H}}$ such that $\widehat{\partial}-\partial \in \mathcal{H}$ and the adjoint action of $\widehat{\partial}$ on $\mathcal{H}$ is a scalar multiple of $\mathcal{E}$. The next lemma will allow us to find $\widehat{\partial}$ explicitly.

Lemma 3.6. For all $k=1, \ldots, 2 N$, we have:

$$
\widetilde{\partial}^{k}=\partial^{k}-\frac{1}{2} \chi\left(\partial^{k}\right) \mathcal{E}+\iota_{*}\left(x^{k} e^{-\chi} \otimes_{H} e\right)-\operatorname{ad}^{\mathfrak{s p}} \partial^{k}+\sum_{1 \leq i<j \leq 2 N} c_{i j}^{k} f^{i j} \bmod \mathcal{W}_{1}
$$

where we use the notation (2.9), (3.7) and (3.30).

Proof. Start with (2.48):

$$
\widetilde{\partial}^{k}=\partial^{k}+1 \otimes \partial^{k}-\operatorname{ad} \partial^{k} \bmod \mathcal{W}_{1}
$$

From Lemma 3.4, we know that

$$
\begin{aligned}
\iota_{*}\left(x^{k} e^{-\chi} \otimes_{H} e\right) & =e^{-\chi} \otimes \partial^{k}-\sum_{1 \leq i<j \leq 2 N} c_{i j}^{k} x^{j} e^{-\chi} \otimes \partial^{i} \bmod \mathcal{W}_{1} \\
& =1 \otimes \partial^{k}-\chi \otimes \partial^{k}-\sum_{1 \leq i<j \leq 2 N} c_{i j}^{k} x^{j} \otimes \partial^{i} \bmod \mathcal{W}_{1} .
\end{aligned}
$$

By Lemma 2.4, $\chi \otimes \partial^{k} \bmod \mathcal{W}_{1}$ is identified with $-\partial^{k} \otimes \chi \in \mathfrak{d} \otimes \mathfrak{d}^{*} \simeq \mathfrak{g l} \mathfrak{d}$, and $x^{j} \otimes$ $\partial^{i} \bmod \mathcal{W}_{1}$ with $-\partial^{i} \otimes x^{j}=-e^{i j} \in \mathfrak{g l} \mathfrak{d}$. Hence,

$$
1 \otimes \partial^{k}=\iota_{*}\left(x^{k} e^{-\chi} \otimes_{H} e\right)-\partial^{k} \otimes \chi-\sum_{1 \leq i<j \leq 2 N} c_{i j}^{k} e^{i j} \bmod \mathcal{W}_{1}
$$


which gives

$$
\widetilde{\partial}^{k}=\partial^{k}-\operatorname{ad} \partial^{k}+\iota_{*}\left(x^{k} e^{-\chi} \otimes_{H} e\right)-\partial^{k} \otimes \chi-\sum_{1 \leq i<j \leq 2 N} c_{i j}^{k} e^{i j} \bmod \mathcal{W}_{1} .
$$

Using the definition of $\operatorname{ad}^{\mathfrak{s p}}$ in (3.30), we rewrite the right-hand side as

$$
\partial^{k}+\iota_{*}\left(x^{k} e^{-\chi} \otimes_{H} e\right)-\operatorname{ad}^{\mathfrak{s p}} \partial^{k}-\sum_{1 \leq i<j \leq 2 N} c_{i j}^{k} e^{i j}+\frac{1}{2} \sum_{i, j=1}^{2 N} c_{i j}^{k} e^{i j}-\frac{1}{2} \chi\left(\partial^{k}\right) I \quad \bmod \mathcal{W}_{1}
$$

The proof then follows from the identity

$$
\sum_{i, j=1}^{2 N} c_{i j}^{k} e^{i j}=\sum_{1 \leq i<j \leq 2 N} c_{i j}^{k}\left(e^{i j}-e^{j i}\right)
$$

the definition $(3.7)$ of $f^{i j}$, and the fact that $\mathcal{E} \bmod \mathcal{W}_{1}$ identifies with $I \in \mathfrak{g l} \mathfrak{d}$.

Corollary 3.2. The above Lie algebra homomorphism $\gamma: \mathfrak{d} \hookrightarrow \mathcal{H}+\mathbf{k} \mathcal{E}$ satisfies

$$
\gamma(\partial)-\frac{1}{2} \chi(\partial) \mathcal{E} \in \mathcal{H}, \quad \partial \in \mathfrak{d}
$$

Proof. Using $\gamma(\partial)=\partial-\widetilde{\partial}$ and $\mathfrak{s p d} \simeq \mathcal{H}_{0} / \mathcal{H}_{1} \simeq\left(\mathcal{H}_{0}+\mathcal{W}_{1}\right) / \mathcal{W}_{1}$, it follows from Lemma 3.6 that

$$
\gamma(\partial)-\frac{1}{2} \chi(\partial) \mathcal{E} \in \mathcal{H}+\mathcal{W}_{1}
$$

As the projection of $\mathcal{E}$ to $\mathcal{W}_{0} / \mathcal{W}_{1} \simeq \mathfrak{g l} \mathfrak{d}$ does not belong to $\mathfrak{s p} \mathfrak{d}$, we see that $\left(\mathcal{H}+\mathcal{W}_{1}\right) \cap$ $(\mathcal{H}+\mathbf{k} \mathcal{E})=\mathcal{H}$.

As a consequence of Corollary 3.2, we have

$$
\widehat{\partial}=\widetilde{\partial}+\frac{1}{2} \chi(\partial) \mathcal{E} \in \partial+\mathcal{H} \subset \widetilde{\mathcal{H}}, \quad \partial \in \mathfrak{d} .
$$

Since $\partial \mapsto \widetilde{\partial}$ is Lie algebra isomorphism and $\chi$ is a trace-form, it follows that $\partial \mapsto \widehat{\partial}$ is a Lie algebra isomorphism from $\mathfrak{d}$ onto a subalgebra $\widehat{\mathfrak{d}}$ of $\widetilde{\mathcal{H}}$. The Lie algebra $\widehat{\mathfrak{d}}$ normalizes every $\mathcal{H}_{n}$, as so does $\mathcal{E}$ and $[\widetilde{\mathfrak{d}}, \mathcal{H}]=\{0\}$.

Remark 3.2. Recall that $H(\mathfrak{d}, \chi, \omega) \subset S(\mathfrak{d}, \phi)$, where $\phi=-N \chi+\operatorname{tr}$ ad (see Remark 3.1 and Lemma 3.1). Our elements $\widehat{\partial}$ coincide with those for $S(\mathfrak{d}, \phi)$ defined in [2, (3.29)], if we replace there $\chi$ by $\phi$ and $N$ by $2 N=\operatorname{dim} \mathfrak{d}$.

Now we can determine the normalizers of $\mathcal{H}_{0}$ in $\widetilde{\mathcal{H}}$ and of $\mathcal{P}_{0}$ in $\widetilde{\mathcal{P}}$. 
Proposition 3.2. For $n \geq 0$, the normalizer $\mathcal{N}_{\mathcal{H}}$ of $\mathcal{H}_{n}$ inside $\widetilde{\mathcal{H}}$ coincides with $\widehat{\mathfrak{d}} \ltimes \mathcal{H}_{0}$ and is independent of $n$. There is a decomposition as a direct sum of vector spaces $\widetilde{\mathcal{H}}=\mathfrak{d} \oplus \mathcal{N}_{\mathcal{H}}$.

Proof. Both $\mathcal{H}_{0}$ and $\widehat{\mathfrak{d}}$ normalize $\mathcal{H}_{n}$. If we denote by $\mathcal{N}_{\mathcal{H}}$ the subspace $\widehat{\mathfrak{d}}+\mathcal{H}_{0}=\widehat{\mathfrak{d}} \ltimes \mathcal{H}_{0}$, then we obtain a direct sum of vector spaces $\widetilde{\mathcal{H}}=\mathfrak{d} \oplus \mathcal{N}_{\mathcal{H}}$. Since $\mathcal{H}_{n} \varsubsetneqq \partial\left(\mathcal{H}_{n}\right) \subset \mathcal{H}_{n-1}$ for all $0 \neq \partial \in \mathfrak{d}$, we see that no element outside $\mathcal{N}_{\mathcal{H}}$ is contained in the normalizer of $\mathcal{H}_{n}$ in $\widetilde{\mathcal{H}}$, thus proving the statement.

Recall that the Lie algebra homomorphism $\iota_{*}: \widetilde{\mathcal{P}} \rightarrow \widetilde{\mathcal{H}}$ has a kernel $\mathbf{k} e^{-\chi}$, which is the center of $\mathcal{P}$. Hence, $\iota_{*}^{-1}(\widehat{\mathfrak{d}})$ is a subalgebra of $\widetilde{\mathcal{P}}$, as $\widehat{\mathfrak{d}}$ is a subalgebra of $\widetilde{\mathcal{H}}$. Since $\mathcal{P}_{0}$ is stabilized by $\iota_{*}^{-1}(\widehat{\mathfrak{d}})$, we have the semidirect sum

$$
\mathcal{N}_{\mathcal{P}}:=\iota_{*}^{-1}\left(\mathcal{N}_{\mathcal{H}}\right)=\iota_{*}^{-1}(\widehat{\mathfrak{d}}) \ltimes \mathcal{P}_{0}
$$

Proposition 3.3. For $n \geq 0$, the normalizer of $\mathcal{P}_{n}$ inside $\widetilde{\mathcal{P}}$ coincides with $\mathcal{N}_{\mathcal{P}}$ and is independent of $n$. There is a decomposition as a direct sum of vector spaces $\widetilde{\mathcal{P}}=\mathfrak{d} \oplus \mathcal{N}_{\mathcal{P}}$.

Proof. Since it is a Lie algebra homomorphism, $\iota_{*}$ sends elements of $\widetilde{\mathcal{P}}$ normalizing $\mathcal{P}_{n}$ to elements of $\widetilde{\mathcal{H}}$ normalizing $\mathcal{H}_{n}$. Therefore, the normalizer of $\mathcal{P}_{n}$ inside $\widetilde{\mathcal{P}}$ is contained in $\iota_{*}^{-1}\left(\mathcal{N}_{\mathcal{H}}\right)=\mathcal{N}_{\mathcal{P}}$.

We already know that $\left[\mathcal{P}_{0}, \mathcal{P}_{n}\right] \subset \mathcal{P}_{n}$. Moreover, from $\left[\mathfrak{d}, \mathcal{P}_{n}\right] \subset \mathcal{P}_{n-1}$ and $\left[\mathcal{P}, \mathcal{P}_{n}\right] \subset$ $\mathcal{P}_{n-1}$ we deduce $\left[\iota_{*}^{-1}(\widehat{\mathfrak{d}}), \mathcal{P}_{n}\right] \subset \mathcal{P}_{n-1}$. Since $\left[\widehat{\mathfrak{d}}, \mathcal{H}_{n}\right] \subset \mathcal{H}_{n}$, we also obtain $\left[\iota_{*}^{-1}(\widehat{\mathfrak{d}}), \mathcal{P}_{n}\right] \subset$ $\mathcal{P}_{n}+\mathbf{k} e^{-\chi}$. However, $e^{-\chi} \notin \mathcal{P}_{m}$ for $m \geq-1$, which implies $\left[\iota_{*}^{-1}(\widehat{\mathfrak{d}}), \mathcal{P}_{n}\right] \subset \mathcal{P}_{n}$ if $n \geq 0$. Finally, the fact that $\widetilde{\mathcal{P}}=\mathfrak{d} \oplus \mathcal{N}_{\mathcal{P}}$ is clear.

Lemma 3.7. The Lie algebra $\iota_{*}^{-1}(\widehat{\mathfrak{d}})$ is an abelian extension of $\widehat{\mathfrak{d}} \simeq \mathfrak{d}$ by the ideal $\mathbf{k} e^{-\chi}$ of character $\chi$ with cocycle $\omega$. This means that in $\iota_{*}^{-1}(\widehat{\mathfrak{d}})$ :

$$
\left[\widehat{\partial}, e^{-\chi}\right]=\chi(\partial) e^{-\chi}, \quad\left[\widehat{\partial}, \widehat{\partial}^{\prime}\right]=\widehat{\left[\partial, \partial^{\prime}\right]}+\omega\left(\partial \wedge \partial^{\prime}\right) e^{-\chi}, \quad \partial, \partial^{\prime} \in \mathfrak{d} .
$$

Proof. Let us identify elements $\widehat{\partial} \in \widehat{\mathfrak{d}} \subset \widetilde{\mathcal{H}}$ with their unique liftings to $\mathfrak{d} \ltimes \mathcal{P}_{-1} \subset \widetilde{\mathcal{P}}$. By (2.16), we have in $\widetilde{\mathcal{P}}$ :

$$
\left[\partial, e^{-\chi}\right]=\partial\left(e^{-\chi}\right)=\chi(\partial) e^{-\chi}
$$

Since $\widehat{\partial}-\partial \in \mathcal{H}$ and $\left[\mathcal{H}, e^{-\chi}\right]=0$, we get $\left[\widehat{\partial}, e^{-\chi}\right]=\chi(\partial) e^{-\chi}$.

To prove the second identity in (3.36), notice that

$$
\left[\widehat{\partial}^{i}, \widehat{\partial}^{j}\right]-\widehat{\left[\partial, \partial^{\prime}\right]} \in \operatorname{ker} \iota_{*}=\mathbf{k} e^{-\chi}
$$

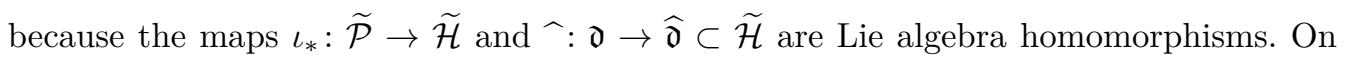
the other hand, by (3.34) and Lemma 3.6, 


$$
\left[\widehat{\partial}^{i}, \widehat{\partial}^{j}\right] \in\left[\partial^{i}+x^{i} e^{-\chi}+\mathcal{P}_{0}, \partial^{j}+x^{j} e^{-\chi}+\mathcal{P}_{0}\right]
$$

The only terms giving contributions in $\mathbf{k} e^{-\chi}$ are:

$$
\left[\partial^{i}, x^{j} e^{-\chi}\right], \quad\left[\partial^{j}, x^{i} e^{-\chi}\right], \quad\left[x^{i} e^{-\chi}, x^{j} e^{-\chi}\right]
$$

and the coefficients of $e^{-\chi}$ can be found by taking images in $X / \mathrm{F}_{0} X \simeq \mathbf{k}$. All three are easy to compute. For instance, using again (2.16), we obtain

$$
\begin{aligned}
{\left[\partial^{i}, x^{j} e^{-\chi}\right] } & =\partial^{i}\left(x^{j} e^{-\chi}\right)=\left(\partial^{i} x^{j}+\chi\left(\partial^{i}\right) x^{j}\right) e^{-\chi} \\
& =\partial^{i} x^{j} \bmod \mathrm{F}_{0} X=-r^{i j} \bmod \mathrm{F}_{0} X
\end{aligned}
$$

by (3.4) and (2.12). Similarly, using (3.24), (2.15) and (2.13),

$$
\begin{aligned}
{\left[x^{i} e^{-\chi}, x^{j} e^{-\chi}\right] } & \left.=\sum_{k=1}^{2 N}\left(\left(x^{i} e^{-\chi}\right) \bar{\partial}_{k}\right)\right)\left(\left(x^{j} e^{-\chi}\right) \bar{\partial}^{k}\right) \\
& =\sum_{k=1}^{2 N}\left(x^{i} \partial_{k}\right)\left(x^{j} \partial^{k}\right) e^{-2 \chi} \\
& =r^{i j} \bmod \mathrm{F}_{0} X
\end{aligned}
$$

Adding all contributions, we obtain

$$
\left[\widehat{\partial}^{i}, \widehat{\partial}^{j}\right]=-r^{i j} e^{-\chi} \bmod \widehat{\mathfrak{d}}
$$

which gives

$$
\left[\widehat{\partial}_{i}, \widehat{\partial}_{j}\right]=\omega_{i j} e^{-\chi} \bmod \widehat{\mathfrak{d}}
$$

after lowering indices (see (3.4), (3.5)).

\subsection{Finite-dimensional irreducible representations of $\left.\iota_{*}^{-1} \widehat{\mathfrak{d}}\right)$}

In this subsection, we briefly study the finite-dimensional irreducible representations of the Lie algebra $\mathfrak{d}^{\prime}:=\iota_{*}^{-1}(\widehat{\mathfrak{d}})$. They will be used later in the classification of irreducible representations of $H(\mathfrak{d}, \chi, \omega)$.

Lemma 3.8. Let $V$ be a finite-dimensional irreducible representation of $\mathfrak{d}^{\prime}$. Then $e^{-\chi}$ acts trivially on $V$ unless $\chi=0$ and $\omega$ is a 2-coboundary.

Proof. Recall that the structure of the Lie algebra $\mathfrak{d}^{\prime}$ is described in Lemma 3.7. In particular, $c=e^{-\chi}$ spans an abelian ideal of $\mathfrak{d}^{\prime}$, hence it lies in the radical. By the CartanJacobson Theorem, $c$ must act via scalar multiplication by some element $\lambda \in \mathbf{k}$ on each 
finite-dimensional irreducible representation of $\mathfrak{d}^{\prime}$. If $c \in\left[\mathfrak{d}^{\prime}, \mathfrak{d}^{\prime}\right]$, then $\operatorname{tr}_{V} c=0=\lambda \operatorname{dim} V$ and $\lambda=0$.

Suppose that $c \notin\left[\mathfrak{d}^{\prime}, \mathfrak{d}^{\prime}\right]$. Then, by (3.36), we have $\chi=0$ and $\mathfrak{d}^{\prime}$ is a central extension of $\mathfrak{d}$ with a center $\mathbf{k} c$ and a 2-cocycle $\omega$. Let $\overline{\mathfrak{d}}$ be any subspace of $\mathfrak{d}^{\prime}$ containing $\left[\mathfrak{d}^{\prime}, \mathfrak{d}^{\prime}\right]$, such that $\mathfrak{d}^{\prime}=\overline{\mathfrak{d}} \oplus \mathbf{k} c$ as vector spaces. Then $[\overline{\mathfrak{d}}, \overline{\mathfrak{d}}] \subset\left[\mathfrak{d}^{\prime}, \mathfrak{d}^{\prime}\right] \subset \overline{\mathfrak{d}}$, so $\overline{\mathfrak{d}}$ is a subalgebra of $\mathfrak{d}^{\prime}$. Therefore, the central extension splits and $\omega$ is a 2 -coboundary.

When $\omega$ is a 2 -coboundary, we may find a 1 -form $\zeta$ of $\mathfrak{d}$ (i.e., $\zeta \in \mathfrak{d}^{*}$ ) such that $\mathrm{d} \zeta=\omega$. Then elements $\widehat{\partial}+\zeta(\partial) e^{-\chi}$ with $\partial \in \mathfrak{d}$ span a Lie subalgebra $\mathfrak{d} \zeta \subset \mathfrak{d}^{\prime}$ isomorphic to $\mathfrak{d}$, which provides a splitting $\mathfrak{d}^{\prime}=\mathfrak{d}^{\zeta} \oplus \mathbf{k} e^{-\chi}$. As a consequence, we obtain:

Proposition 3.4. Let $V$ be a finite-dimensional irreducible representation of $\mathfrak{d}^{\prime}$. Then:

- either $V$ is obtained by lifting to $\mathfrak{d}^{\prime}$ an irreducible representation of $\mathfrak{d} \simeq \mathfrak{d}^{\prime} / \mathbf{k} e^{-\chi}$, in which case $e^{-\chi}$ acts trivially on $V$;

- or $\chi=0, \omega=\mathrm{d} \zeta$, so that $\mathfrak{d}^{\prime}$ splits as a direct sum of Lie algebras $\mathfrak{d}^{\prime}=\mathfrak{d}^{\zeta} \oplus \mathbf{k} 1$, and $V$ is obtained from an irreducible representation of $\mathfrak{d}^{\zeta} \simeq \mathfrak{d}$ by supplementing it with a (possibly non-trivial) scalar action of the center $\mathbf{k} 1$.

\section{Tensor modules for $H(\mathfrak{d}, \chi, \omega)$}

In our classification of irreducible finite $W(\mathfrak{d})$-modules [2], a crucial role was played by the so-called tensor modules. In this section, by restricting them to the subalgebra $H(\mathfrak{d}, \chi, \omega)$ of $W(\mathfrak{d})$, we obtain the notion of tensor $H(\mathfrak{d}, \chi, \omega)$-modules.

\subsection{Tensor modules for $W(\mathfrak{d})$}

Let us review the construction of tensor modules for $W(\mathfrak{d})$ from [2]. First, given a Lie algebra $\mathfrak{g}$, we define the semidirect sum $W(\mathfrak{d}) \ltimes$ Cur $\mathfrak{g}$ as a direct sum as $H$-modules, for which $W(\mathfrak{d})$ is a subalgebra and Cur $\mathfrak{g}$ is an ideal, with the following pseudobracket between them:

$$
[(f \otimes a) *(g \otimes b)]=-(f \otimes g a) \otimes_{H}(1 \otimes b),
$$

for $f, g \in H, a \in \mathfrak{d}, b \in \mathfrak{g}$ (see Example 2.1 and Section 2.5). For any finite-dimensional $\mathfrak{g}$-module $V_{0}$, we construct a representation of $W(\mathfrak{d}) \ltimes \operatorname{Cur} \mathfrak{g}$ in $V=H \otimes V_{0}$ by

$$
((f \otimes a) \oplus(g \otimes b)) * v=-(f \otimes a) \otimes_{H} v+(g \otimes 1) \otimes_{H} b v,
$$

where $f, g \in H, a \in \mathfrak{d}, b \in \mathfrak{g}, v \in V_{0}$. For convenience, in (4.1), we identify $v \in V_{0}$ with its image $1 \otimes v \in \mathbf{k} \otimes V_{0} \subset V$. The action on $V_{0}$ is then extended to the whole $V$ by $H$-linearity. Note that (4.1) combines the usual action of Cur $\mathfrak{g}$ on $V$ from Example 2.2 with the $W(\mathfrak{d})$-action on $H$ given by $(2.40)$. 
Next, we define an embedding of $W(\mathfrak{d})$ in $W(\mathfrak{d}) \ltimes \operatorname{Cur}(\mathfrak{d} \oplus \mathfrak{g l} \mathfrak{d})$ by

$$
1 \otimes \partial_{i} \mapsto\left(1 \otimes \partial_{i}\right) \oplus\left(\left(1 \otimes \partial_{i}\right) \oplus\left(1 \otimes \operatorname{ad} \partial_{i}+\sum_{j} \partial_{j} \otimes e_{i}^{j}\right)\right)
$$

Composing this embedding with the action (4.1) of $W(\mathfrak{d}) \ltimes \operatorname{Cur} \mathfrak{g}$ for $\mathfrak{g}=\mathfrak{d} \oplus \mathfrak{g l} \mathfrak{d}$, we obtain a $W(\mathfrak{d})$-module $V=H \otimes V_{0}$ for each $(\mathfrak{d} \oplus \mathfrak{g l} \mathfrak{d})$-module $V_{0}$. This module is called a tensor $W(\mathfrak{d})$-module and is denoted $\mathcal{T}\left(V_{0}\right)$. Explicitly, the action of $W(\mathfrak{d})$ on $\mathcal{T}\left(V_{0}\right)$ is given by [2, Eq. (4.30)]:

$$
\begin{aligned}
\left(1 \otimes \partial_{i}\right) * v & =(1 \otimes 1) \otimes_{H}\left(\operatorname{ad} \partial_{i}\right) v+\sum_{j}\left(\partial_{j} \otimes 1\right) \otimes_{H} e_{i}^{j} v \\
& -\left(1 \otimes \partial_{i}\right) \otimes_{H} v+(1 \otimes 1) \otimes_{H}\left(\partial_{i} \cdot v\right),
\end{aligned}
$$

for $v \in V_{0}$. Here we denoted by $\partial_{i} \cdot v \in V_{0}$ the action of $\partial_{i} \in \mathfrak{d}$ on $v \in V_{0}$, which should be distinguished from the element $\partial_{i} \otimes v_{0} \in V$.

If $\Pi$ is a finite-dimensional $\mathfrak{d}$-module and $U$ is a finite-dimensional $\mathfrak{g l} \mathfrak{d}$-module, their exterior tensor product $\Pi \otimes U$ is defined as the $(\mathfrak{d} \oplus \mathfrak{g l} \mathfrak{d})$-module $\Pi \otimes U$, where $\mathfrak{d}$ acts on the first factor and $\mathfrak{g l} \mathfrak{d}$ acts on the second one. In this case, the tensor module $\mathcal{T}(\Pi \otimes U)$ will also be denoted as $\mathcal{T}(\Pi, U)$. Notice that

$$
\mathcal{T}(\Pi, U)=T_{\Pi}(\mathcal{T}(\mathbf{k}, U))
$$

where $T_{\Pi}$ is the twisting functor from Section 2.3. More generally, given two $\mathfrak{d}$-modules $\Pi_{1}, \Pi_{2}$ and a $\mathfrak{g l} \mathfrak{d}$-module $U$, we have

$$
T_{\Pi_{1}}\left(\mathcal{T}\left(\Pi_{2}, U\right)\right)=\mathcal{T}\left(\Pi_{1} \otimes \Pi_{2}, U\right)
$$

\subsection{Restriction of tensor modules from $W(\mathfrak{d})$ to $H(\mathfrak{d}, \chi, \omega)$}

We will identify $H(\mathfrak{d}, \chi, \omega)$ as a subalgebra of $W(\mathfrak{d})$ via the embedding (3.17). Then $H(\mathfrak{d}, \chi, \omega)=H e$ where $e \in W(\mathfrak{d})$ is given by (3.20). In view of (4.2), we introduce the $H$-linear map (but not a homomorphism of Lie pseudoalgebras) $\tau: W(\mathfrak{d}) \rightarrow$ Cur $\mathfrak{g l} \mathfrak{d}$ given by

$$
\tau\left(h \otimes \partial_{i}\right)=h \otimes \operatorname{ad} \partial_{i}+\sum_{j=1}^{2 N} h \partial_{j} \otimes e_{i}^{j}, \quad h \in H
$$

Then the image of $e$ under the map (4.2) is $e \oplus(e \oplus \tau(e))$, where the first $e$ is considered an element of $W(\mathfrak{d})=H \otimes \mathfrak{d}$, while the second $e$ is in Cur $\mathfrak{d}=H \otimes \mathfrak{d}$.

Recall also the notation $\bar{\partial}=\partial-\chi(\partial)$ and the linear map $\operatorname{ad}^{\mathfrak{s p}}: \mathfrak{d} \rightarrow \mathfrak{s p} \mathfrak{d}$ defined by (3.30). Then we have the following analog of [3, Lemma 5.1]. 
Lemma 4.1. We have

$$
\begin{aligned}
\tau(e) & =\left(\operatorname{id} \otimes \operatorname{ad}^{\mathfrak{s p}}\right)(e)+\frac{1}{2} s \otimes I+\sum_{i, j=1}^{2 N} \bar{\partial}_{i} \bar{\partial}_{j} \otimes f^{i j} \\
& =-\sum_{k=1}^{2 N} \bar{\partial}_{k} \otimes\left(\operatorname{ad}^{\mathfrak{s p}} \partial^{k}+\frac{1}{2} \chi\left(\partial^{k}\right) I\right)+\sum_{i, j=1}^{2 N} \bar{\partial}_{i} \bar{\partial}_{j} \otimes f^{i j}
\end{aligned}
$$

Proof. First note that, by (3.3), (3.4) and (4.6),

$$
\tau\left(h \otimes \partial^{i}\right)=h \otimes \operatorname{ad} \partial^{i}+\sum_{j=1}^{2 N} h \partial_{j} \otimes e^{i j}, \quad h \in H
$$

Applying this to (3.20), we find

$$
\begin{aligned}
\tau(e) & =-\sum_{i=1}^{2 N} \bar{\partial}_{i} \otimes \operatorname{ad} \partial^{i}-\sum_{i, j=1}^{2 N} \bar{\partial}_{i} \partial_{j} \otimes e^{i j} \\
& =-\sum_{i=1}^{2 N} \bar{\partial}_{i} \otimes\left(\operatorname{ad} \partial^{i}+\sum_{j=1}^{2 N} \chi\left(\partial_{j}\right) e^{i j}\right)-\sum_{i, j=1}^{2 N} \bar{\partial}_{i} \bar{\partial}_{j} \otimes e^{i j} \\
& =-\sum_{k=1}^{2 N} \bar{\partial}_{k} \otimes\left(\operatorname{ad} \partial^{k}+\partial^{k} \otimes \chi\right)-\sum_{i, j=1}^{2 N} \bar{\partial}_{i} \bar{\partial}_{j} \otimes e^{i j}
\end{aligned}
$$

Now we write

$$
\begin{gathered}
-\sum_{i, j=1}^{2 N} \bar{\partial}_{i} \bar{\partial}_{j} \otimes e^{i j}=-\frac{1}{2} \sum_{i, j=1}^{2 N} \bar{\partial}_{i} \bar{\partial}_{j} \otimes e^{i j}-\frac{1}{2} \sum_{i, j=1}^{2 N} \bar{\partial}_{j} \bar{\partial}_{i} \otimes e^{j i} \\
=-\frac{1}{2} \sum_{i, j=1}^{2 N} \bar{\partial}_{i} \bar{\partial}_{j} \otimes e^{i j}-\frac{1}{2} \sum_{i, j=1}^{2 N} \bar{\partial}_{i} \bar{\partial}_{j} \otimes e^{j i}+\frac{1}{2} \sum_{i, j=1}^{2 N}\left[\bar{\partial}_{i}, \bar{\partial}_{j}\right] \otimes e^{j i} \\
=\sum_{i, j=1}^{2 N} \bar{\partial}_{i} \bar{\partial}_{j} \otimes f^{i j}-\frac{1}{2} \sum_{i, j=1}^{2 N}\left[\bar{\partial}_{i}, \bar{\partial}_{j}\right] \otimes e^{i j}
\end{gathered}
$$

Since the map (2.14) is a Lie algebra homomorphism, (2.9) gives

$$
\left[\bar{\partial}_{i}, \bar{\partial}_{j}\right]=\sum_{k=1}^{2 N} c_{i j}^{k} \bar{\partial}_{k}
$$

Finally, from (3.18), we obtain $(\mathrm{id} \otimes \chi)(e)=\bar{s}=s$. Plugging all of these in the above formula for $\tau(e)$, we derive (4.7). 
As a consequence, we obtain the image of $e$ under the map (4.2). Then, by (4.1), the action of $e$ on a tensor $W(\mathfrak{d})$-module $\mathcal{T}\left(V_{0}\right)$ is given explicitly by:

$$
\begin{aligned}
e * v & =\sum_{k=1}^{2 N}\left(\bar{\partial}_{k} \otimes \partial^{k}\right) \otimes_{H} v-\sum_{k=1}^{2 N}\left(\bar{\partial}_{k} \otimes 1\right) \otimes_{H}\left(\partial^{k}+\operatorname{ad}^{\mathfrak{s p}} \partial^{k}+\frac{1}{2} \chi\left(\partial^{k}\right) I\right) \cdot v \\
& +\sum_{i, j=1}^{2 N}\left(\bar{\partial}_{i} \bar{\partial}_{j} \otimes 1\right) \otimes_{H} f^{i j} v,
\end{aligned}
$$

for $v \in V_{0} \equiv \mathbf{k} \otimes V_{0} \subset \mathcal{T}\left(V_{0}\right)$.

Remark 4.1. We already know that the unique inclusion $H(\mathfrak{d}, \chi, \omega) \hookrightarrow W(\mathfrak{d})$ induces a non-injective homomorphism of the corresponding annihilation algebras, its kernel being spanned over $\mathbf{k}$ by the central element $c=e^{-\chi} \in \mathcal{P}$. Consequently, every module obtained from a $W(\mathfrak{d})$-tensor module by restricting it to $H(\mathfrak{d}, \chi, \omega)$ necessarily exhibits a trivial action of $c$. One may provide an alternate construction which allows for nonzero scalar actions of the center of $\mathcal{P}$ as follows.

Assume that $\chi=0$ and $\omega=\mathrm{d} \zeta$ (cf. Lemma 3.8). Set $H^{\prime}=U\left(\mathfrak{d}^{\prime}\right)$ and $X^{\prime}=\left(H^{\prime}\right)^{*}$, where $\mathfrak{d}^{\prime}=\iota_{*}^{-1}(\widehat{\mathfrak{d}})$ as before. Recall that we may construct, as in [1, Remark 8.23], a Lie pseudoalgebra $K\left(\mathfrak{d}^{\prime}, \theta\right)=H^{\prime} e^{\prime}$, whose annihilation algebra we denote by $\mathcal{K}$. Then the canonical projection of Lie algebras $\pi: \mathfrak{d}^{\prime} \rightarrow \mathfrak{d}^{\prime} / \mathbf{k} c \simeq \mathfrak{d}$ induces corresponding algebra homomorphisms $\pi_{*}: H^{\prime} \rightarrow H$ and $\pi^{*}: X \rightarrow X^{\prime}$ so that the map

$$
\mathcal{P} \rightarrow \mathcal{K}, \quad x \otimes_{H} e \mapsto \pi^{*}(x) \otimes_{H^{\prime}} e^{\prime}
$$

is an injective Lie algebra homomorphism. As both the left and the right action of $\partial_{0}=c=e^{-\chi}$ are trivial on $\pi^{*}(X)$, one obtains that all Fourier coefficients $\pi^{*}(x) \otimes_{H^{\prime}} \partial_{0} e^{\prime}$ vanish, and that the semidirect product $\mathfrak{d}^{\prime} \ltimes \mathcal{P}$ induced from the extended annihilation algebra $\mathfrak{d}^{\prime} \ltimes \mathcal{K}$ projects to $\mathfrak{d} \ltimes \mathcal{P}$.

Due to the correspondence between representations of a Lie pseudoalgebra and conformal representations of the corresponding extended annihilation algebra, one may consider tensor modules for $K\left(\mathfrak{d}^{\prime}, \theta\right)$, introduced in [3], and restrict the action of $\mathcal{K}$ to the subalgebra $\mathcal{P}$. One thus obtains a representation $T$ of the semidirect product $\mathfrak{d}^{\prime} \ltimes \mathcal{P}$, and it is not difficult to check that $T / \partial_{0} T$ then admits an action of $\mathfrak{d} \ltimes \mathcal{P}$, thus yielding a representation of $H(\mathfrak{d}, \chi, \omega)$ with a possibly non-trivial action of $e^{-\chi} \in \mathcal{P}$.

\subsection{Tensor modules for $H(\mathfrak{d}, \chi, \omega)$}

For any trace form $\varphi$ on $\mathfrak{d}$, the corresponding 1-dimensional $\mathfrak{d}$-module $\mathbf{k}_{\varphi}$ is spanned by an element $1_{\varphi}$ such that $\partial \cdot 1_{\varphi}=\varphi(\partial) 1_{\varphi}$ for $\partial \in \mathfrak{d}$. Similarly, for any $\mathfrak{d}$-module $\Pi$, 
we may form the tensor product $\mathfrak{d}$-module $\Pi_{\varphi}:=\Pi \otimes \mathbf{k}_{\varphi}$, which is isomorphic to $\Pi$ as a vector space, but is endowed with the action

$$
\partial \cdot\left(u \otimes 1_{\varphi}\right)=((\partial+\varphi(\partial)) \cdot u) \otimes 1_{\varphi}, \quad \partial \in \mathfrak{d}, \quad u \in \Pi
$$

This observation implies that, by changing the action of $\mathfrak{d}$ on a $(\mathfrak{d} \oplus \mathfrak{g l} \mathfrak{d})$-module $V_{0}$, we can eliminate the term $\frac{1}{2} \chi\left(\partial^{k}\right) I$ in (4.8). Furthermore, note that both $\operatorname{ad}^{\mathfrak{s p}} \partial^{k}$ and $f^{i j}$ lie in the subalgebra $\mathfrak{s p} \mathfrak{d} \subset \mathfrak{g l} \mathfrak{d}$. This motivates the following definition.

Definition 4.1. Let $V_{0}$ be a finite-dimensional representation of $\mathfrak{d}^{\prime} \oplus \mathfrak{s p} \mathfrak{d}$. Then the tensor module $\mathcal{T}\left(V_{0}\right)$ over $H(\mathfrak{d}, \chi, \omega)$ is defined as $\mathcal{T}\left(V_{0}\right)=H \otimes V_{0}$ with the action:

$$
\begin{aligned}
e * v & =\sum_{k=1}^{2 N}\left(\bar{\partial}_{k} \otimes \partial^{k}\right) \otimes_{H} v-\sum_{k=1}^{2 N}\left(\bar{\partial}_{k} \otimes 1\right) \otimes_{H}\left(\partial^{k}+\operatorname{ad}^{\mathfrak{s p}} \partial^{k}\right) \cdot v+(1 \otimes 1) \otimes_{H}(c \cdot v) \\
& +\sum_{i, j=1}^{2 N}\left(\bar{\partial}_{i} \bar{\partial}_{j} \otimes 1\right) \otimes_{H}\left(f^{i j} \cdot v\right), \quad v \in V_{0} \equiv \mathbf{k} \otimes V_{0} \subset \mathcal{T}\left(V_{0}\right)
\end{aligned}
$$

where $c=e^{-\chi}$. For $V_{0}=\Pi^{\prime} \otimes U$, where $\Pi^{\prime}$ is a finite-dimensional $\mathfrak{d}^{\prime}$-module and $U$ is a finite-dimensional $\mathfrak{s p} \mathfrak{d}$-module, we will write $\mathcal{T}\left(V_{0}\right)=\mathcal{T}\left(\Pi^{\prime}, U\right)$. When $V_{0}=\Pi \otimes U$ is a $\mathfrak{d} \oplus$ $\mathfrak{s p} \mathfrak{d}$-module, we will abuse the notation and set $\mathcal{T}\left(V_{0}\right)=\mathcal{T}(\Pi, U):=\mathcal{T}\left(\pi^{*} \Pi, U\right)$, where $\pi^{*} \Pi$ is viewed as a $\mathfrak{d}^{\prime}$-module via composition with the canonical projection $\pi: \mathfrak{d}^{\prime} \rightarrow$ $\mathfrak{d}^{\prime} / \mathbf{k} c \simeq \mathfrak{d}$.

As usual, the action of $H(\mathfrak{d}, \chi, \omega)=H e$ on $\mathcal{T}\left(V_{0}\right)$ is determined uniquely from (4.10) and $H$-linearity. Notice that when the action of $c \in \mathfrak{d}^{\prime}$ is trivial and $\Pi^{\prime}$ gets identified with $\pi^{*} \Pi$ as above, (4.10) coincides with the expression from (4.8), thus showing that $\mathcal{T}\left(V_{0}\right)$ is indeed an $H(\mathfrak{d}, \chi, \omega)$-module. One may in principle use Remark 4.1 to generalize this fact to non-trivial actions of $c$; we will instead postpone this to Section 6 , where it will be derived in terms of singular vectors. Using (4.8)-(4.10), we obtain the next proposition.

Proposition 4.1. Let $\Pi$ be a finite-dimensional $\mathfrak{d}$-module, and $U$ be a finite-dimensional $\mathfrak{g l} \mathfrak{d}$-module, which is also an $\mathfrak{s p d}$-module by restriction. Suppose that $I \in \mathfrak{g l} \mathfrak{d}$ acts as $k$ id on $U$ for some $k \in \mathbf{k}$. Then the restriction of the tensor $W(\mathfrak{d})$-module $\mathcal{T}(\Pi, U)$ to the subalgebra $H(\mathfrak{d}, \chi, \omega)$ is isomorphic to the tensor $H(\mathfrak{d}, \chi, \omega)$-module $\mathcal{T}\left(\Pi_{k \chi / 2}, U\right)$.

\section{Tensor modules of de Rham type}

The main goal of this section is to define an important complex of $H(\mathfrak{d}, \chi, \omega)$-modules, called the conformally symplectic pseudo de Rham complex. We start by reviewing the pseudo de Rham complex of $W(\mathfrak{d})$-modules from $[1,2]$. 


\subsection{Forms with constant coefficients}

Consider the cohomology complex of the Lie algebra $\mathfrak{d}$ with trivial coefficients:

$$
0 \rightarrow \Omega^{0} \stackrel{\mathrm{d}_{0}}{\longrightarrow} \Omega^{1} \stackrel{\mathrm{d}_{0}}{\longrightarrow} \cdots \stackrel{\mathrm{d}_{0}}{\longrightarrow} \Omega^{2 N} \rightarrow 0, \quad \operatorname{dim} \mathfrak{d}=2 N
$$

where $\Omega^{n}=\bigwedge^{n} \mathfrak{d}^{*}$. Set $\Omega=\bigwedge^{\bullet} \mathfrak{d}^{*}=\bigoplus_{n=0}^{2 N} \Omega^{n}$ and $\Omega^{n}=\{0\}$ if $n<0$ or $n>2 N$. We will think of the elements of $\Omega^{n}$ as skew-symmetric $n$-forms, i.e., linear maps from $\bigwedge^{n} \mathfrak{d}$ to $\mathbf{k}$. The differential $\mathrm{d}_{0}$ is given by the formula $\left(\alpha \in \Omega^{n}, a_{i} \in \mathfrak{d}\right)$ :

$$
\begin{aligned}
& \left(\mathrm{d}_{0} \alpha\right)\left(a_{1} \wedge \cdots \wedge a_{n+1}\right) \\
& \quad=\sum_{i<j}(-1)^{i+j} \alpha\left(\left[a_{i}, a_{j}\right] \wedge a_{1} \wedge \cdots \wedge \widehat{a}_{i} \wedge \cdots \wedge \widehat{a}_{j} \wedge \cdots \wedge a_{n+1}\right), \quad n \geq 1,
\end{aligned}
$$

and $\mathrm{d}_{0} \alpha=0$ if $\alpha \in \Omega^{0}=\mathbf{k}$. Here, as usual, a hat over a term means that it is omitted in the wedge product. Recall also that the wedge product of two forms $\alpha \in \Omega^{n}$ and $\beta \in \Omega^{p}$ is defined by:

$$
\begin{aligned}
(\alpha & \wedge \beta)\left(a_{1} \wedge \cdots \wedge a_{n+p}\right) \\
& =\frac{1}{n ! p !} \sum_{\pi \in S_{n+p}}(\operatorname{sgn} \pi) \alpha\left(a_{\pi(1)} \wedge \cdots \wedge a_{\pi(n)}\right) \beta\left(a_{\pi(n+1)} \wedge \cdots \wedge a_{\pi(n+p)}\right),
\end{aligned}
$$

where $S_{n+p}$ denotes the symmetric group on $n+p$ letters and $\operatorname{sgn} \pi$ is the sign of the permutation $\pi$.

The wedge product (5.3) makes $\Omega$ a $\mathbb{Z}_{+}$-graded associative commutative superalgebra with parity $p(\alpha)=n \bmod 2 \mathbb{Z}$ for $\alpha \in \Omega^{n}$ :

$$
\alpha \wedge \beta=(-1)^{p(\alpha) p(\beta)} \beta \wedge \alpha, \quad(\alpha \wedge \beta) \wedge \gamma=\alpha \wedge(\beta \wedge \gamma)
$$

The differential $\mathrm{d}_{0}$ is an odd derivation of $\Omega$ :

$$
\mathrm{d}_{0}(\alpha \wedge \beta)=\mathrm{d}_{0} \alpha \wedge \beta+(-1)^{p(\alpha)} \alpha \wedge \mathrm{d}_{0} \beta
$$

For $a \in \mathfrak{d}$, we have operators $\iota_{a}: \Omega^{n} \rightarrow \Omega^{n-1}$ defined by

$$
\left(\iota_{a} \alpha\right)\left(a_{1} \wedge \cdots \wedge a_{n-1}\right)=\alpha\left(a \wedge a_{1} \wedge \cdots \wedge a_{n-1}\right), \quad a_{i} \in \mathfrak{d}
$$

which are also odd derivations of $\Omega$. For $A \in \mathfrak{g l} \mathfrak{d}$, denote by $A$. its action on $\Omega$ :

$$
(A \cdot \alpha)\left(a_{1} \wedge \cdots \wedge a_{n}\right)=\sum_{i=1}^{n}(-1)^{i} \alpha\left(A a_{i} \wedge a_{1} \wedge \cdots \wedge \widehat{a}_{i} \wedge \cdots \wedge a_{n}\right) .
$$


Then $A \cdot$ is an even derivation of $\Omega$ :

$$
A \cdot(\alpha \wedge \beta)=(A \cdot \alpha) \wedge \beta+\alpha \wedge(A \cdot \beta)
$$

As before, let us fix a 1-form $\chi \in \Omega^{1}=\mathfrak{d}^{*}$ and a nondegenerate 2-form $\omega \in \Omega^{2}=$ $\mathfrak{d}^{*} \wedge \mathfrak{d}^{*}$. Then equation (3.11) and the condition that $\chi$ is a trace form are equivalent to:

$$
\mathrm{d}_{0} \chi=0, \quad \mathrm{~d}_{0} \omega+\chi \wedge \omega=0 .
$$

Consider the operator $\Psi$ of wedge multiplication with $\omega$, i.e., $\Psi(\alpha)=\omega \wedge \alpha$ for $\alpha \in \Omega$. Denote by $I^{n} \subset \Omega^{n}$ the image of $\left.\Psi\right|_{\Omega^{n-2}}$ and by $J^{n} \subset \Omega^{n}$ the kernel of $\left.\Psi\right|_{\Omega^{n}}$. Since $\omega$ is nondegenerate, we have:

$$
I^{k}=\Omega^{k} \text { for } k \geq N+1, \quad J^{n}=\{0\} \text { for } n \leq N-1
$$

In particular, $\Psi$ gives an isomorphism $\Omega^{N-1} \rightarrow \Omega^{N+1}$. More generally, $\Psi^{m}$ gives an isomorphism $\Omega^{N-m} \rightarrow \Omega^{N+m}$ for $0 \leq m \leq N$. By [3, Lemma 6.1], the composition

$$
J^{N+m} \hookrightarrow \Omega^{N+m} \stackrel{\simeq}{\longrightarrow} \Omega^{N-m} \rightarrow \Omega^{N-m} / I^{N-m}
$$

is an isomorphism for $0 \leq m \leq N$.

By definition, $A \cdot \omega=0$ for $A \in \mathfrak{s p d}$. Hence, by (5.8), $A$. commutes with $\Psi$. This implies that $I^{n}$ and $J^{n}$ are $\mathfrak{s p} \mathfrak{d}$-modules and (5.11) is an isomorphism of $\mathfrak{s p} \mathfrak{d}$-modules. In fact, one has isomorphisms of $\mathfrak{s p} \mathfrak{d}$-modules

$$
\Omega^{n} / I^{n} \simeq J^{2 N-n} \simeq R\left(\pi_{n}\right), \quad 0 \leq n \leq N
$$

where $R\left(\pi_{n}\right), 1 \leq n \leq N$, denotes the $n$-th fundamental representation of $\mathfrak{s p} \mathfrak{d}$ and $R\left(\pi_{0}\right)=\mathbf{k}$ (see, e.g., [7, Lecture 17]).

\subsection{Pseudo de Rham complex}

Recall from [1] the spaces of pseudoforms

$$
\Omega^{n}(\mathfrak{d})=H \otimes \Omega^{n}, \quad \Omega(\mathfrak{d})=H \otimes \Omega=\bigoplus_{n=0}^{2 N} \Omega^{n}(\mathfrak{d})
$$

These are free $H$-modules, where $H$ acts by left multiplication on the first factor. We will often identify $n$-pseudoforms $\alpha \in \Omega^{n}(\mathfrak{d})$ with $\mathbf{k}$-linear maps from $\bigwedge^{n} \mathfrak{d}^{*}$ to $H$. The differential $\mathrm{d}: \Omega^{n}(\mathfrak{d}) \rightarrow \Omega^{n+1}(\mathfrak{d})$ is given by $\left(\alpha \in \Omega^{n}(\mathfrak{d}), a_{i} \in \mathfrak{d}\right)$ : 


$$
\begin{aligned}
& (\mathrm{d} \alpha)\left(a_{1} \wedge \cdots \wedge a_{n+1}\right) \\
& \quad=\sum_{i<j}(-1)^{i+j} \alpha\left(\left[a_{i}, a_{j}\right] \wedge a_{1} \wedge \cdots \wedge \widehat{a}_{i} \wedge \cdots \wedge \widehat{a}_{j} \wedge \cdots \wedge a_{n+1}\right) \\
& \quad+\sum_{i}(-1)^{i} \alpha\left(a_{1} \wedge \cdots \wedge \widehat{a}_{i} \wedge \cdots \wedge a_{n+1}\right) a_{i} \in H \quad \text { if } n \geq 1, \\
& (\mathrm{~d} \alpha)\left(a_{1}\right)=-\alpha a_{1} \in H \quad \text { if } \quad \alpha \in \Omega^{0}(\mathfrak{d})=H .
\end{aligned}
$$

Notice that $\mathrm{d}$ is $H$-linear and $\mathrm{d}^{2}=0$. The sequence

$$
0 \rightarrow \Omega^{0}(\mathfrak{d}) \stackrel{\mathrm{d}}{\rightarrow} \Omega^{1}(\mathfrak{d}) \stackrel{\mathrm{d}}{\rightarrow} \cdots \stackrel{\mathrm{d}}{\rightarrow} \Omega^{2 N-1}(\mathfrak{d}) \stackrel{\mathrm{d}}{\rightarrow} \Omega^{2 N}(\mathfrak{d})
$$

is called the pseudo de Rham complex. By [1, Remark 8.1], the $n$-th cohomology of the complex $(\Omega(\mathfrak{d}), \mathrm{d})$ is trivial for $n \neq 2 N=\operatorname{dim} \mathfrak{d}$ and is 1-dimensional for $n=2 N$. Hence, the sequence (5.15) is exact. We extend the wedge product in $\Omega$ to a product in $\Omega(\mathfrak{d})$ by setting

$$
(f \otimes \alpha) \wedge(g \otimes \beta)=(f g) \otimes(\alpha \wedge \beta), \quad \alpha, \beta \in \Omega, \quad f, g \in H
$$

Then, by [3, Lemma 6.3], we have:

$$
\mathrm{d}(\alpha \wedge \beta)=\mathrm{d}_{0} \alpha \wedge \beta+(-1)^{n} \alpha \wedge \mathrm{d} \beta, \quad \alpha \in \Omega^{n}, \quad \beta \in \Omega .
$$

In [1,2], we introduced a $W(\mathfrak{d})$-module structure on each $\Omega^{n}(\mathfrak{d})$, so that the map $\mathrm{d}$ is a module homomorphism. Furthermore, $\Omega^{n}(\mathfrak{d})$ is a tensor $W(\mathfrak{d})$-module, namely $\Omega^{n}(\mathfrak{d})=\mathcal{T}\left(\mathbf{k}, \Omega^{n}\right)$ (see Section 4.1). Explicitly, if we identify the elements of $H^{\otimes 2} \otimes_{H}$ $\Omega^{n}(\mathfrak{d}) \simeq H^{\otimes 2} \otimes \Omega^{n}$ with k-linear maps from $\bigwedge^{n} \mathfrak{d}^{*}$ to $H^{\otimes 2}$, the action of $W(\mathfrak{d})$ on $\Omega^{n}(\mathfrak{d})$ is given by:

$$
\begin{aligned}
((f \otimes a) & * \alpha)\left(a_{1} \wedge \cdots \wedge a_{n}\right)=-\alpha\left(a_{1} \wedge \cdots \wedge a_{n}\right)(f \otimes a) \\
& +\sum_{i=1}^{n}(-1)^{i} \alpha\left(a \wedge a_{1} \wedge \cdots \wedge \widehat{a}_{i} \wedge \cdots \wedge a_{n}\right)\left(f a_{i} \otimes 1\right) \\
& +\sum_{i=1}^{n}(-1)^{i} \alpha\left(\left[a, a_{i}\right] \wedge a_{1} \wedge \cdots \wedge \widehat{a}_{i} \wedge \cdots \wedge a_{n}\right)(f \otimes 1) \in H^{\otimes 2}
\end{aligned}
$$

for $n \geq 1, f \otimes a \in W(\mathfrak{d})$ and $\alpha \in \Omega^{n} \equiv \mathbf{k} \otimes \Omega^{n} \subset \Omega^{n}(\mathfrak{d})$. For $n=0$, the action of $W(\mathfrak{d})$ on $\Omega^{0}(\mathfrak{d})=H$ coincides with the action $(2.40)$.

\subsection{Conformally symplectic pseudo de Rham complex for $\chi=0$}

In this subsection, we will assume that $\chi=0$. The general case will be considered in the next subsection. Let us extend $\Psi$ by $H$-linearity to a homomorphism of $H$-modules 
$\Psi: \Omega^{n}(\mathfrak{d}) \rightarrow \Omega^{n+2}(\mathfrak{d})$. Then the image of $\left.\Psi\right|_{\Omega^{n-2}(\mathfrak{d})}$ is $I^{n}(\mathfrak{d})=H \otimes I^{n} \subset \Omega^{n}(\mathfrak{d})$, and the kernel of $\left.\Psi\right|_{\Omega^{n}(\mathfrak{d})}$ is $J^{n}(\mathfrak{d})=H \otimes J^{n} \subset \Omega^{n}(\mathfrak{d})$. By (5.11), we have isomorphisms $J^{N+m}(\mathfrak{d}) \simeq \Omega^{N-m}(\mathfrak{d}) / I^{N-m}(\mathfrak{d})$ for all $0 \leq m \leq N$.

It follows from (5.17) and (5.9) with $\chi=0$ that $\mathrm{d} \Psi=\Psi \mathrm{d}$. Therefore, the complex (5.15) induces complexes

$$
0 \rightarrow \Omega^{0}(\mathfrak{d}) / I^{0}(\mathfrak{d}) \stackrel{\mathrm{d}}{\rightarrow} \Omega^{1}(\mathfrak{d}) / I^{1}(\mathfrak{d}) \stackrel{\mathrm{d}}{\rightarrow} \cdots \stackrel{\mathrm{d}}{\rightarrow} \Omega^{N}(\mathfrak{d}) / I^{N}(\mathfrak{d})
$$

and

$$
J^{N}(\mathfrak{d}) \stackrel{\mathrm{d}}{\rightarrow} J^{N+1}(\mathfrak{d}) \stackrel{\mathrm{d}}{\rightarrow} \cdots \stackrel{\mathrm{d}}{\rightarrow} J^{2 N}(\mathfrak{d}) \rightarrow 0
$$

Lemma 5.1. The sequences (5.19) and

$$
J^{N}(\mathfrak{d}) \stackrel{\mathrm{d}}{\rightarrow} J^{N+1}(\mathfrak{d}) \stackrel{\mathrm{d}}{\rightarrow} \cdots \stackrel{\mathrm{d}}{\rightarrow} J^{2 N-2}(\mathfrak{d}) \stackrel{\mathrm{d}}{\rightarrow} J^{2 N-1}(\mathfrak{d}), \quad N \geq 3,
$$

are exact. The cohomology of the complex (5.20) is finite dimensional.

Proof. To show exactness at the term $\Omega^{n}(\mathfrak{d}) / I^{n}(\mathfrak{d})$ in (5.19) for $0 \leq n \leq N-1$, take $\alpha \in \Omega^{n}(\mathfrak{d})$ such that $\mathrm{d} \alpha \in I^{n+1}(\mathfrak{d})$. This means $\mathrm{d} \alpha=\Psi \beta$ for some $\beta \in \Omega^{n-1}(\mathfrak{d})$. Then $0=\mathrm{d}^{2} \alpha=\mathrm{d} \Psi \beta=\Psi \mathrm{d} \beta$; hence, $\mathrm{d} \beta=0$, because $\Psi$ is injective on $\Omega^{n}(\mathfrak{d})$ for $n \leq N-1$. By the exactness of (5.15), $\beta=\mathrm{d} \gamma$ for some $\gamma \in \Omega^{n-2}(\mathfrak{d})$. Then $\mathrm{d}(\alpha-\Psi \gamma)=\Psi \beta-\Psi \mathrm{d} \gamma=0$, which implies $\alpha-\Psi \gamma=\mathrm{d} \rho$ for some $\rho \in \Omega^{n-1}(\mathfrak{d})$. Therefore, $\alpha+I^{n}(\mathfrak{d})=\mathrm{d}\left(\rho+I^{n-1}(\mathfrak{d})\right)$ is a coboundary.

To prove exactness at the term $J^{n}(\mathfrak{d})$ in (5.20) for $N+1 \leq n \leq 2 N-2$, take $\alpha \in J^{n}(\mathfrak{d})$ such that $\mathrm{d} \alpha=0$. Then $\alpha=\mathrm{d} \beta$ for some $\beta \in \Omega^{n-1}(\mathfrak{d})$, by the exactness of (5.15). We have $\mathrm{d} \Psi \beta=\Psi \mathrm{d} \beta=\Psi \alpha=0$. Hence, $\Psi \beta=\mathrm{d} \gamma$ for some $\gamma \in \Omega^{n}(\mathfrak{d})$, since $\Psi \beta \in \Omega^{n+1}(\mathfrak{d})$ with $n+1 \leq 2 N-1$ and (5.15) is exact. Since $\Psi$ maps $\Omega^{n-2}(\mathfrak{d})$ onto $\Omega^{n}(\mathfrak{d})$ for $n \geq N+1$, we can find $\rho \in \Omega^{n-2}(\mathfrak{d})$ such that $\Psi \rho=\gamma$. Then $\Psi(\beta-\mathrm{d} \rho)=\Psi \beta-\mathrm{d} \Psi \rho=\Psi \beta-\mathrm{d} \gamma=0$. We have found an element $\beta-\mathrm{d} \rho \in J^{n-1}(\mathfrak{d})$ such that $\mathrm{d}(\beta-\mathrm{d} \rho)=\alpha$. This completes the proof of exactness.

The finite-dimensionality of the cohomology of (5.20) follows from the fact that $\operatorname{dim} \Omega^{2 N}(\mathfrak{d}) / \mathrm{d} \Omega^{2 N-1}(\mathfrak{d})=1$.

Now we will construct a map $\mathrm{d}^{\mathrm{R}}: \Omega^{N}(\mathfrak{d}) / I^{N}(\mathfrak{d}) \rightarrow J^{N}(\mathfrak{d})$, analogous to Rumin's map from [11] and [3]. Take any $\alpha \in \Omega^{N}(\mathfrak{d})$. Since the restriction of $\Psi$ gives an isomorphism $\Omega^{N-1}(\mathfrak{d}) \rightarrow \Omega^{N+1}(\mathfrak{d})$, we can write $\mathrm{d} \alpha=\Psi \beta$ for a unique $\beta \in \Omega^{N-1}(\mathfrak{d})$. Then we have $0=\mathrm{d}^{2} \alpha=\Psi \mathrm{d} \beta$; hence, $\mathrm{d} \beta \in J^{N}(\mathfrak{d})$. We let $\mathrm{d}^{\mathrm{R}} \alpha=\mathrm{d} \beta$. To show that $\mathrm{d}^{\mathrm{R}}$ is well defined, we need to check that $\mathrm{d}^{\mathrm{R}} \alpha=0$ when $\alpha \in I^{N}(\mathfrak{d})$. Indeed, if $\alpha=\Psi \gamma$ for some $\gamma \in \Omega^{N-2}(\mathfrak{d})$, then $\mathrm{d} \alpha=\Psi \mathrm{d} \gamma$, which implies $\beta=\mathrm{d} \gamma$ and $\mathrm{d} \beta=0$. Connecting (5.19) and (5.20), we obtain a sequence

$$
0 \rightarrow \Omega^{0}(\mathfrak{d}) / I^{0}(\mathfrak{d}) \stackrel{\mathrm{d}}{\rightarrow} \cdots \stackrel{\mathrm{d}}{\rightarrow} \Omega^{N}(\mathfrak{d}) / I^{N}(\mathfrak{d}) \stackrel{\mathrm{d}^{\mathrm{R}}}{\rightarrow} J^{N}(\mathfrak{d}) \stackrel{\mathrm{d}}{\rightarrow} \cdots \stackrel{\mathrm{d}}{\rightarrow} J^{2 N}(\mathfrak{d})
$$


which we will call the conformally symplectic pseudo de Rham complex for $\chi=0$. (Since for $\chi=0$ the form $\omega$ is symplectic, one could also call this the symplectic pseudo de Rham complex.)

Proposition 5.1. The sequence (5.21) is a complex, which is exact at all terms except $J^{2 N-1}(\mathfrak{d})$ and $J^{2 N}(\mathfrak{d})$. The map $\mathrm{d}^{\mathrm{R}}$ is defined as follows: take any representative $\alpha \in$ $\Omega^{N}(\mathfrak{d})$ and write $\mathrm{d} \alpha=\Psi \beta$; then $\mathrm{d}^{\mathrm{R}} \alpha=\mathrm{d} \beta$.

Proof. We have already shown that $\mathrm{d}^{\mathrm{R}}$ is well defined. Next, it is clear by construction that $\mathrm{d}^{\mathrm{R}} \mathrm{d}=0$ and $\mathrm{dd}^{\mathrm{R}}=0$. Due to Lemma 5.1, it remains only to check exactness at the terms $\Omega^{N}(\mathfrak{d}) / I^{N}(\mathfrak{d})$ and $J^{N}(\mathfrak{d})$, the latter only for $N \geq 2$.

Let $\alpha \in \Omega^{N}(\mathfrak{d})$ be such that $\mathrm{d}^{\mathrm{R}} \alpha=0$. Then $\mathrm{d} \beta=\mathrm{d}^{\mathrm{R}} \alpha=0$; hence, $\beta=\mathrm{d} \gamma$ for some $\gamma \in \Omega^{N-2}(\mathfrak{d})$ by the exactness of (5.15). Then $\mathrm{d} \alpha=\Psi \beta=\mathrm{d} \Psi \gamma$ implies that $\alpha-\Psi \gamma=\mathrm{d} \rho$ for some $\rho \in \Omega^{N-1}(\mathfrak{d})$. Therefore, $\alpha+I^{N}(\mathfrak{d})=\mathrm{d}\left(\rho+I^{N-1}(\mathfrak{d})\right)$.

Now suppose that $N \geq 2$ and $\alpha \in J^{N}(\mathfrak{d})$ satisfies $\mathrm{d} \alpha=0$. Then $\alpha=\mathrm{d} \beta$ for some $\beta \in$ $\Omega^{N-1}(\mathfrak{d})$, again by the exactness of (5.15). We have $\mathrm{d} \Psi \beta=\Psi \alpha=0$. Since $\Psi \beta \in \Omega^{N+1}(\mathfrak{d})$ and $N+1 \leq 2 N-1$, there is $\gamma \in \Omega^{N}(\mathfrak{d})$ such that $\Psi \beta=\mathrm{d} \gamma$. Then $\mathrm{d}^{\mathrm{R}} \gamma=\mathrm{d} \beta=\alpha$, as desired.

Recall that, by [2], $\Omega^{n}(\mathfrak{d})=\mathcal{T}\left(\mathbf{k}, \Omega^{n}\right)$ is a tensor $W(\mathfrak{d})$-module (see Section 4.1). Since $I \in \mathfrak{g l} \mathfrak{d}$ acts on $\Omega^{n}$ as $-n$ id, Proposition 4.1 tells us that, considered as an $H(\mathfrak{d}, \chi, \omega)$-module, $\Omega^{n}(\mathfrak{d})$ is isomorphic to the tensor module $\mathcal{T}\left(\mathbf{k}_{-n \chi / 2}, \Omega^{n}\right)$. Then its quotient $\Omega^{n}(\mathfrak{d}) / I^{n}(\mathfrak{d})$ and submodule $J^{n}(\mathfrak{d})$ are also tensor $H(\mathfrak{d}, \chi, \omega)$-modules, because $\Omega^{n}(\mathfrak{d}) / I^{n}(\mathfrak{d})=H \otimes\left(\Omega^{n} / I^{n}\right)$ and $J^{n}(\mathfrak{d})=H \otimes J^{n}$. From (5.12), we obtain isomorphisms of $H(\mathfrak{d}, \chi, \omega)$-modules:

$$
\begin{aligned}
\Omega^{n}(\mathfrak{d}) / I^{n}(\mathfrak{d}) & \simeq \mathcal{T}\left(\mathbf{k}_{-n \chi / 2}, R\left(\pi_{n}\right)\right), \\
J^{2 N-n}(\mathfrak{d}) & \simeq \mathcal{T}\left(\mathbf{k}_{-(2 N-n) \chi / 2}, R\left(\pi_{n}\right)\right), \quad 0 \leq n \leq N
\end{aligned}
$$

Furthermore, the differential $\mathrm{d}$ in (5.15) is a homomorphism of $W(\mathfrak{d})$-modules. Hence, $\mathrm{d}$ in $(5.21)$ is a homomorphism of $H(\mathfrak{d}, \chi, \omega)$-modules. We will prove in the next subsection that $\mathrm{d}^{\mathrm{R}}$ is a homomorphism too, and will generalize all these results to the case $\chi \neq 0$.

\subsection{Twisted conformally symplectic pseudo de Rham complex}

Now let us consider the general case, i.e., $\chi$ is not necessarily 0 . When $\chi \neq 0$, the maps d and $\Psi$ no longer commute. Accordingly, we will modify the definition of $\Psi$ using the twisting functors from Section 2.3.

Fix a finite-dimensional $\mathfrak{d}$-module $\Pi$. Applying the twisting functor $T_{\Pi}$ to the pseudo de Rham complex (5.15), we obtain the exact complex

$$
0 \rightarrow \Omega_{\Pi}^{0}(\mathfrak{d}) \stackrel{\mathrm{d}_{\Pi}}{\longrightarrow} \Omega_{\Pi}^{1}(\mathfrak{d}) \stackrel{\mathrm{d}_{\Pi}}{\longrightarrow} \cdots \stackrel{\mathrm{d}_{\Pi}}{\longrightarrow} \Omega_{\Pi}^{2 N}(\mathfrak{d})
$$


where

$$
\mathrm{d}_{\Pi}=T_{\Pi}(\mathrm{d}), \quad \Omega_{\Pi}^{n}(\mathfrak{d})=T_{\Pi}\left(\Omega^{n}(\mathfrak{d})\right)=H \otimes \Pi \otimes \Omega^{n}
$$

As before, $H$ acts on $\Omega_{\Pi}^{n}(\mathfrak{d})$ by left multiplication on the first factor. As a $W(\mathfrak{d})$-module, $\Omega_{\Pi}^{n}(\mathfrak{d})=\mathcal{T}\left(\Pi, \Omega^{n}\right)$ is a tensor module.

Recall that $\mathbf{k}_{\chi}=\mathbf{k} 1_{\chi}$ where $\partial \cdot 1_{\chi}=\chi(\partial) 1_{\chi}$ for $\partial \in \mathfrak{d}$, and $\Pi_{\chi}$ denotes the $\mathfrak{d}$-module $\Pi \otimes \mathbf{k}_{\chi}$. We introduce $H$-linear maps

$$
\Psi_{\chi}: \Omega_{\Pi}^{n}(\mathfrak{d}) \rightarrow \Omega_{\Pi_{\chi}}^{n+2}(\mathfrak{d}), \quad h \otimes u \otimes \alpha \mapsto h \otimes u \otimes 1_{\chi} \otimes(\omega \wedge \alpha)
$$

where $h \in H, u \in \Pi, \alpha \in \Omega^{n}$. We claim that these maps are compatible with the differentials.

Lemma 5.2. With the above notation (5.26), we have $\Psi_{\chi} \mathrm{d}_{\Pi}=\mathrm{d}_{\Pi_{\chi}} \Psi_{\chi}$.

Proof. By $H$-linearity, it is enough to show that both sides of the desired identity are equal when applied to $1 \otimes u \otimes \alpha \in \Omega_{\Pi}^{n}(\mathfrak{d})$ for $u \in \Pi, \alpha \in \Omega^{n}$. Furthermore, we will assume that $\alpha=\iota_{\partial_{i}} \beta$ for some $\beta \in \Omega^{n+1}$ and $1 \leq i \leq 2 N$, as such elements span $\Omega^{n}$. Note that, by $[2$, Lemma 5.1],

$$
\mathrm{d} \alpha \in \sum_{k=1}^{2 N} \partial_{k} \otimes\left(e_{i}^{k} \cdot \beta\right)+\mathbf{k} \otimes \Omega^{n+1} \subset \Omega^{n+1}(\mathfrak{d})
$$

Then from the definition $(2.31)$ of $T_{\Pi}$, we obtain

$$
\mathrm{d}_{\Pi}(1 \otimes u \otimes \alpha)=\sigma_{12}(u \otimes \mathrm{d} \alpha)-\sum_{k=1}^{2 N} 1 \otimes\left(\partial_{k} \cdot u\right) \otimes\left(e_{i}^{k} \cdot \beta\right)
$$

where $\sigma_{12}: \Pi \otimes H \otimes \Omega^{n} \rightarrow H \otimes \Pi \otimes \Omega^{n}$ is the transposition of the first two factors. (This equation can also be derived from [2, Lemma 5.3].) Hence,

$$
\begin{aligned}
& \Psi_{\chi} \mathrm{d}_{\Pi}(1 \otimes u \otimes \alpha) \\
& \quad=\sigma_{12}\left(\left(u \otimes 1_{\chi}\right) \otimes(\omega \wedge \mathrm{d} \alpha)\right)-\sum_{k=1}^{2 N} 1 \otimes\left(\partial_{k} \cdot u\right) \otimes 1_{\chi} \otimes\left(\omega \wedge\left(e_{i}^{k} \cdot \beta\right)\right),
\end{aligned}
$$

where now $\sigma_{12}: \Pi_{\chi} \otimes H \otimes \Omega^{n} \rightarrow H \otimes \Pi_{\chi} \otimes \Omega^{n}$.

On the other hand,

$$
\Psi_{\chi}(1 \otimes u \otimes \alpha)=1 \otimes u \otimes 1_{\chi} \otimes(\omega \wedge \alpha)
$$

and by (5.9), (5.17), we have 


$$
\mathrm{d}(\omega \wedge \alpha)=\mathrm{d}_{0} \omega \wedge \alpha+\omega \wedge \mathrm{d} \alpha=-\chi \wedge \omega \wedge \alpha+\omega \wedge \mathrm{d} \alpha
$$

This and (5.27) imply

$$
\mathrm{d}(\omega \wedge \alpha) \in \sum_{k=1}^{2 N} \partial_{k} \otimes\left(\omega \wedge e_{i}^{k} \cdot \beta\right)+\mathbf{k} \otimes \Omega^{n+3} \subset \Omega^{n+3}(\mathfrak{d})
$$

Using again (2.31) and (5.28), we get

$$
\begin{aligned}
\mathrm{d}_{\Pi_{\chi}}\left(1 \otimes u \otimes 1_{\chi} \otimes(\omega \wedge \alpha)\right) & \\
= & \sigma_{12}\left(\left(u \otimes 1_{\chi}\right) \otimes \mathrm{d}(\omega \wedge \alpha)\right)-\sum_{k=1}^{2 N} 1 \otimes \partial_{k} \cdot\left(u \otimes 1_{\chi}\right) \otimes\left(\omega \wedge\left(e_{i}^{k} \cdot \beta\right)\right) \\
& =\sigma_{12}\left(\left(u \otimes 1_{\chi}\right) \otimes(\omega \wedge \mathrm{d} \alpha)\right)-\sum_{k=1}^{2 N} 1 \otimes\left(\partial_{k} \cdot u\right) \otimes 1_{\chi} \otimes\left(\omega \wedge\left(e_{i}^{k} \cdot \beta\right)\right) \\
& -\sigma_{12}\left(\left(u \otimes 1_{\chi}\right) \otimes(\chi \wedge \omega \wedge \alpha)\right)-\sum_{k=1}^{2 N} 1 \otimes u \otimes\left(\partial_{k} \cdot 1_{\chi}\right) \otimes\left(\omega \wedge\left(e_{i}^{k} \cdot \beta\right)\right) .
\end{aligned}
$$

In order to finish the proof of the lemma, we need to show that the last two terms in the right-hand side cancel. In other words, we claim that

$$
\sum_{k=1}^{2 N} \chi\left(\partial_{k}\right) e_{i}^{k} \cdot \beta=-\chi \wedge\left(\iota_{\partial_{i}} \beta\right), \quad \beta \in \Omega^{n+1}
$$

Indeed, evaluating either side on $\partial_{j_{1}} \wedge \cdots \wedge \partial_{j_{n+1}}$ gives

$$
\sum_{t=1}^{n+1}(-1)^{t} \chi\left(\partial_{j_{t}}\right) \beta\left(\partial_{i} \wedge \partial_{j_{1}} \wedge \cdots \wedge \widehat{\partial_{j_{t}}} \wedge \cdots \wedge \partial_{j_{n+1}}\right)
$$

by using (5.3), (5.6) and (5.7).

Lemma 5.3. The maps $\Psi_{\chi}: \Omega_{\Pi}^{n}(\mathfrak{d}) \rightarrow \Omega_{\Pi_{\chi}}^{n+2}(\mathfrak{d})$, defined by (5.26), are homomorphisms of $H(\mathfrak{d}, \chi, \omega)$-modules.

Proof. By $H$-linearity, it is enough to show that $\Psi_{\chi}$ commutes with the action of $e$ on $1 \otimes u \otimes \alpha$, where $u \in \Pi, \alpha \in \Omega^{n}$ and $e \in H(\mathfrak{d}, \chi, \omega) \subset W(\mathfrak{d})$ is the generator (3.20). Since $\Omega_{\Pi}^{n}(\mathfrak{d})=\mathcal{T}\left(\Pi, \Omega^{n}\right)=\mathcal{T}\left(V_{0}\right)$ is a tensor $W(\mathfrak{d})$-module, the action of $e$ on it is given by (4.8) for $v=u \otimes \alpha \in V_{0}=\Pi \otimes \Omega^{n}$.

We have:

$$
I \cdot \alpha=-n \alpha, \quad I \cdot(\omega \wedge \alpha)=-(n+2)(\omega \wedge \alpha), \quad \alpha \in \Omega^{n},
$$




$$
\partial \cdot\left(u \otimes 1_{\chi}\right)=(\partial \cdot u) \otimes 1_{\chi}+\chi(\partial) u \otimes 1_{\chi}, \quad \partial \in \mathfrak{d}, \quad u \in \Pi,
$$

and, by (5.8),

$$
A \cdot(\omega \wedge \alpha)=(A \cdot \omega) \wedge \alpha+\omega \wedge(A \cdot \alpha)=\omega \wedge(A \cdot \alpha), \quad A \in \mathfrak{s p \mathfrak { d }}
$$

Plugging these into (4.8), we get:

$$
\begin{aligned}
& e *\left(\Psi_{\chi}(1 \otimes u \otimes \alpha)\right)-\left((\mathrm{id} \otimes \mathrm{id}) \otimes_{H} \Psi_{\chi}\right)(e *(1 \otimes u \otimes \alpha)) \\
& \quad=e *\left(1 \otimes u \otimes 1_{\chi} \otimes(\omega \wedge \alpha)\right)-\left((\mathrm{id} \otimes \mathrm{id}) \otimes_{H} \Psi_{\chi}\right)(e *(1 \otimes u \otimes \alpha)) \\
& \quad=-\sum_{k=1}^{2 N}\left(\bar{\partial}_{k} \otimes 1\right) \otimes_{H}\left(\chi\left(\partial^{k}\right)+\frac{1}{2} \chi\left(\partial^{k}\right)(-2)\right)\left(1 \otimes u \otimes 1_{\chi} \otimes(\omega \wedge \alpha)\right) \\
& \quad=0 .
\end{aligned}
$$

Therefore, $\Psi_{\chi}$ is an $H(\mathfrak{d}, \chi, \omega)$-module homomorphism.

Notice that the kernel of $\Psi_{\chi}: \Omega_{\Pi}^{n}(\mathfrak{d}) \rightarrow \Omega_{\Pi_{\chi}}^{n+2}(\mathfrak{d})$ is

$$
J_{\Pi}^{n}(\mathfrak{d})=T_{\Pi}\left(J^{n}(\mathfrak{d})\right)=H \otimes \Pi \otimes J^{n} \subset \Omega_{\Pi}^{n}(\mathfrak{d}),
$$

and the image of $\Psi_{-\chi}: \Omega_{\Pi_{\chi}}^{n-2}(\mathfrak{d}) \rightarrow \Omega_{\Pi}^{n}(\mathfrak{d})$ is

$$
I_{\Pi}^{n}(\mathfrak{d})=T_{\Pi}\left(I^{n}(\mathfrak{d})\right)=H \otimes \Pi \otimes I^{n} \subset \Omega_{\Pi}^{n}(\mathfrak{d})
$$

As a consequence of Lemmas 5.2 and 5.3, we obtain complexes of $H(\mathfrak{d}, \chi, \omega)$-modules

$$
0 \rightarrow \Omega_{\Pi}^{0}(\mathfrak{d}) / I_{\Pi}^{0}(\mathfrak{d}) \stackrel{\mathrm{d}_{\Pi}}{\longrightarrow} \Omega^{1}\left(\mathfrak{d}_{\Pi}\right) / I_{\Pi}^{1}(\mathfrak{d}) \stackrel{\mathrm{d}_{\Pi}}{\longrightarrow} \cdots \stackrel{\mathrm{d}_{\Pi}}{\longrightarrow} \Omega_{\Pi}^{N}(\mathfrak{d}) / I_{\Pi}^{N}(\mathfrak{d})
$$

and

$$
J_{\Pi}^{N}(\mathfrak{d}) \stackrel{\mathrm{d}_{\Pi}}{\longrightarrow} J_{\Pi}^{N+1}(\mathfrak{d}) \stackrel{\mathrm{d}_{\Pi}}{\longrightarrow} \cdots \stackrel{\mathrm{d}_{\Pi}}{\longrightarrow} J_{\Pi}^{2 N}(\mathfrak{d}) .
$$

Note that (5.31) and (5.32) are obtained from the complexes of $H(\mathfrak{d}, \chi, \omega)$-modules (5.19) and (5.20) by applying the twisting functor $T_{\Pi}$. Due to (5.22) and (5.23), these are tensor modules:

$$
\begin{aligned}
\Omega_{\Pi}^{n}(\mathfrak{d}) / I_{\Pi}^{n}(\mathfrak{d}) & \simeq \mathcal{T}\left(\Pi_{-n \chi / 2}, R\left(\pi_{n}\right)\right), \\
J_{\Pi}^{2 N-n}(\mathfrak{d}) & \simeq \mathcal{T}\left(\Pi_{-(2 N-n) \chi / 2}, R\left(\pi_{n}\right)\right), \quad 0 \leq n \leq N .
\end{aligned}
$$

In the next lemma, we construct a twisted analog of Rumin's map $\mathrm{d}^{\mathrm{R}}$. 
Lemma 5.4. There is a well-defined $H(\mathfrak{d}, \chi, \omega)$-module homomorphism

$$
\mathrm{d}_{\Pi}^{\mathrm{R}}: \Omega_{\Pi}^{N}(\mathfrak{d}) / I_{\Pi}^{N}(\mathfrak{d}) \rightarrow J_{\Pi_{-\chi}}^{N}(\mathfrak{d})
$$

such that $\mathrm{d}_{\Pi}^{\mathrm{R}} \mathrm{d}_{\Pi}=0$ and $\mathrm{d}_{\Pi_{-\chi}} \mathrm{d}_{\Pi}^{\mathrm{R}}=0$.

Proof. Pick any $\alpha \in \Omega_{\Pi}^{N}(\mathfrak{d})$. Since $\Psi$ gives an isomorphism $\Omega^{N-1} \simeq \Omega^{N+1}$, we can write $\mathrm{d}_{\Pi} \alpha=\Psi_{\chi} \beta$ for a unique $\beta \in \Omega_{\Pi_{-\chi}}^{N-1}(\mathfrak{d})$. By Lemma 5.2, we have

$$
0=\left(\mathrm{d}_{\Pi}\right)^{2} \alpha=\mathrm{d}_{\Pi} \Psi_{\chi} \beta=\Psi_{\chi} \mathrm{d}_{\Pi_{-\chi}} \beta
$$

which means that $\mathrm{d}_{\Pi}^{\mathrm{R}} \alpha:=\mathrm{d}_{\Pi_{-\chi}} \beta \in J_{\Pi_{-\chi}}^{N}(\mathfrak{d})$. To show that $\mathrm{d}_{\Pi}^{\mathrm{R}}$ is well defined, we need to check that $\mathrm{d}_{\Pi}^{\mathrm{R}} \alpha=0$ when $\alpha \in I_{\Pi}^{N}(\mathfrak{d})$. Indeed, if $\alpha=\Psi_{\chi} \gamma$ for some $\gamma \in \Omega_{\Pi_{-\chi}}^{N-2}(\mathfrak{d})$, then

$$
\mathrm{d}_{\Pi} \alpha=\mathrm{d}_{\Pi} \Psi_{\chi} \gamma=\Psi_{\chi} \mathrm{d}_{\Pi_{-\chi}} \gamma
$$

Hence, we can take $\beta=\mathrm{d}_{\Pi_{-\chi}} \gamma$, and we get $\mathrm{d}_{\Pi}^{\mathrm{R}} \alpha=\mathrm{d}_{\Pi_{-\chi}} \beta=0$.

The map $\mathrm{d}_{\Pi}^{\mathrm{R}}$ is a homomorphism of $H(\mathfrak{d}, \chi, \omega)$-modules, because it is a composition of homomorphisms:

$$
\mathrm{d}_{\Pi}^{\mathrm{R}}=\mathrm{d}_{\Pi_{-\chi}}\left(\left.\Psi_{\chi}\right|_{\left.\Omega_{\Pi_{-\chi}-1}^{N-1}\right)}\right)^{-1} \mathrm{~d}_{\Pi}
$$

Finally, the identities $d_{\Pi}^{R} d_{\Pi}=0$ and $d_{\Pi_{-\chi}} d_{\Pi}^{R}=0$ are obvious from (5.35).

Using Lemma 5.4, we can connect the complexes (5.31) and (5.32) to obtain the twisted conformally symplectic pseudo de Rham complex:

$$
\begin{aligned}
0 & \rightarrow \Omega_{\Pi}^{0}(\mathfrak{d}) / I_{\Pi}^{0}(\mathfrak{d}) \stackrel{\mathrm{d}_{\Pi}^{1}}{\longrightarrow} \Omega_{\Pi}^{1}(\mathfrak{d}) / I_{\Pi}^{1}(\mathfrak{d}) \stackrel{\mathrm{d}_{\Pi}^{2}}{\longrightarrow} \cdots \stackrel{\mathrm{d}_{\Pi}^{N}}{\longrightarrow} \Omega_{\Pi}^{N}(\mathfrak{d}) / I_{\Pi}^{N}(\mathfrak{d}) \\
& \stackrel{\mathrm{d}_{\Pi}^{\mathrm{R}}}{\longrightarrow} J_{\Pi_{-\chi}}^{N}(\mathfrak{d}) \stackrel{\mathrm{d}_{\Pi}^{N+1}}{\longrightarrow} J_{\Pi_{-\chi}}^{N+1}(\mathfrak{d}) \stackrel{\mathrm{d}_{\Pi}^{N+2}}{\longrightarrow} \cdots \stackrel{\mathrm{d}_{-\chi}^{2 N}}{\longrightarrow} J_{\Pi_{-\chi}}^{2 N}(\mathfrak{d}),
\end{aligned}
$$

where we decorated the differentials with superscripts that will turn out to be convenient later.

Lemma 5.5. The twisted conformally symplectic pseudo de Rham complex (5.36) is exact at all terms except for $J_{\Pi_{-\chi}}^{2 N-1}(\mathfrak{d})$ and $J_{\Pi_{-\chi}}^{2 N}(\mathfrak{d})$.

Proof. Recall that the twisting functor $T_{\Pi}$ preserves exactness. Hence, the complexes (5.24) and (5.31) are exact, while (5.32) is exact at all terms except the last two (cf. Lemma 5.1). The rest of the proof is the same as in Proposition 5.1.

We summarize the above results in the following theorem. 
Theorem 5.1. For every finite-dimensional $\mathfrak{d}$-module $\Pi$, we have a complex of tensor $H(\mathfrak{d}, \chi, \omega)$-modules

$$
\begin{aligned}
0 & \rightarrow \mathcal{T}\left(\Pi, R\left(\pi_{0}\right)\right) \stackrel{\mathrm{d}_{\Pi}^{1}}{\longrightarrow} \mathcal{T}\left(\Pi_{-\chi / 2}, R\left(\pi_{1}\right)\right) \stackrel{\mathrm{d}_{\Pi}^{2}}{\longrightarrow} \cdots \stackrel{\mathrm{d}_{\Pi}^{N}}{\longrightarrow} \mathcal{T}\left(\Pi_{-N \chi / 2}, R\left(\pi_{N}\right)\right) \\
& \stackrel{\mathrm{d}_{\Pi}^{\mathrm{R}}}{\longrightarrow} \mathcal{T}\left(\Pi_{-(N+2) \chi / 2}, R\left(\pi_{N}\right)\right) \stackrel{\mathrm{d}_{-\chi}^{N+1}}{\longrightarrow} \mathcal{T}\left(\Pi_{-(N+3) \chi / 2}, R\left(\pi_{N-1}\right)\right) \\
& \stackrel{\mathrm{d}_{-\chi}^{N+2}}{\longrightarrow} \cdots \stackrel{\mathrm{d}_{-\chi}^{2 N}}{\longrightarrow} \mathcal{T}\left(\Pi_{-(2 N+2) \chi / 2}, R\left(\pi_{0}\right)\right) \rightarrow 0,
\end{aligned}
$$

where $\mathrm{d}_{\Pi}^{\mathrm{R}}$ is given by (5.35). The complex (5.37) is exact at all terms except for the last two, and its cohomology is finite dimensional.

Proof. The only remaining statement is the finite-dimensionality of the cohomology. This follows from the fact that the cohomology of the complex (5.20) is finite dimensional and the twisting functor $T_{\Pi}$ preserves this property (see Section 2.3).

\section{Singular vectors and tensor modules}

In this section, we introduce the notion of a singular vector in an $H(\mathfrak{d}, \chi, \omega)$-module, describe the action of $H(\mathfrak{d}, \chi, \omega)$ on such vectors, and establish a relationship to tensor modules. We also study the filtration of tensor modules induced by the canonical filtration of $H$.

\subsection{Conformal representations of $\widetilde{\mathcal{P}}$}

Due to Proposition 2.1, any $H(\mathfrak{d}, \chi, \omega)$-module has a natural structure of a conformal $\widetilde{\mathcal{P}}$-module, and vice versa. If $V$ is an irreducible non-trivial $H(\mathfrak{d}, \chi, \omega)$-module, then by Lemma 2.3, each element $v \in V$ lies in some $\operatorname{ker}_{p} V$, which is finite-dimensional and stabilized by the action of $\mathcal{N}_{\mathcal{P}}$. As usual, one has:

Proposition 6.1. The subalgebra $\mathcal{P}_{1} \subset \mathcal{N}_{\mathcal{P}}$ acts trivially on every irreducible finitedimensional conformal $\mathcal{N}_{\mathcal{P}}$-module. Irreducible finite-dimensional conformal $\mathcal{N}_{\mathcal{P}}$-modules are in one-to-one correspondence with irreducible finite-dimensional modules over the Lie algebra $\mathcal{N}_{\mathcal{P}} / \mathcal{P}_{1}=\left(\iota_{*}^{-1}(\widehat{\mathfrak{d}}) \ltimes \mathcal{P}_{0}\right) / \mathcal{P}_{1} \simeq \mathfrak{d}^{\prime} \oplus \mathfrak{s p} \mathfrak{d}$.

Proof. Similar to the proof of [2, Proposition 3.4].

\subsection{Singular vectors}

Here we introduce the notion of a singular vector in an $H(\mathfrak{d}, \chi, \omega)$-module $V$, and we describe the action of $H(\mathfrak{d}, \chi, \omega)$ on such vectors. 
Definition 6.1. A singular vector in $V$ is an element $v \in V$ such that $\mathcal{P}_{1} \cdot v=0$. The space of all singular vectors in $V$ will be denoted by $\operatorname{sing} V$. Note that $\operatorname{sing} V \equiv \operatorname{ker}_{1} V$ is stabilized by the action of $\mathcal{N}_{\mathcal{P}}$, as it normalizes $\mathcal{P}_{1}$. Then

$$
\rho_{\text {sing }}: \mathfrak{d}^{\prime} \oplus \mathfrak{s p} \mathfrak{d} \rightarrow \mathfrak{g l}(\operatorname{sing} V)
$$

will indicate the representation obtained from the $\mathcal{N}_{\mathcal{P}}$-action on $\operatorname{sing} V$ via the isomorphism $\mathcal{N}_{\mathcal{P}} / \mathcal{P}_{1} \simeq \mathfrak{d}^{\prime} \oplus \mathfrak{s p} \mathfrak{d}$, so that

$$
\rho_{\text {sing }}\left(f^{i j}\right) v=\frac{1}{2}\left(x^{i} x^{j} \otimes_{H} e\right) \cdot v, \quad v \in \operatorname{sing} V
$$

whereas $\mathfrak{d}^{\prime}$ is generated over $\mathbf{k}$ by elements $\widehat{\partial}(\partial \in \mathfrak{d})$ and $c=e^{-\chi}$, as in the proof of Lemma 3.7.

Lemma 6.1. A vector $v \in V$ is singular if and only if

$$
e * v \in\left(\mathrm{F}^{2} H \otimes \mathbf{k}\right) \otimes_{H} V
$$

or equivalently,

$$
e * v \in\left(\mathbf{k} \otimes \mathrm{F}^{2} H\right) \otimes_{H} V .
$$

Proof. Analogous statements have been used in both [2] and [3]. Here (6.2) follows from (2.37) and (3.25), whereas the equivalence of (6.2) and (6.3) is a consequence of the straightening technique from [1, Lemma 2.3] and [2, Lemma 2.1(ii)].

Proposition 6.2. Let $V$ be a finite non-zero $H(\mathfrak{d}, \chi, \omega)$-module. Then the vector space $\operatorname{sing} V$ is non-zero and the space $\operatorname{sing} V / \operatorname{ker} V$ is finite dimensional.

Proof. Finite dimensionality of $\operatorname{ker}_{n} V / \operatorname{ker} V$ for all $n$ was stated in Lemma 2.3. In order to prove the other claim, we may assume without loss of generality that $\operatorname{ker} V=\{0\}$. As the $\mathcal{P}$-module $V$ is conformal, then $\operatorname{ker}_{n} V$ is non-zero for some $n \geq 0$. Note that $\operatorname{ker}_{n} V$ is killed by $\mathcal{P}_{n}$, so it is preserved by its normalizer $\mathcal{N}_{\mathcal{P}}$. Choose an irreducible $\mathcal{N}_{\mathcal{P}}$-submodule $U \subset \operatorname{ker}_{n} V$. By Proposition 6.1, the action of $\mathcal{P}_{1}$ on $U$ is trivial; hence, $U \subset \operatorname{sing} V$, which implies $\operatorname{sing} V \neq\{0\}$.

Lemma 6.2. Let $f: M \rightarrow N$ be a homomorphism of $H(\mathfrak{d}, \chi, \omega)$-modules. Then $f(\operatorname{sing} M) \subset \operatorname{sing} N$.

Proof. The proof is obvious, since $f$ commutes with the action of $\mathcal{P}_{1}$.

Lemma 6.3. Let $f: M \rightarrow N$ be a homomorphism of $H(\mathfrak{d}, \chi, \omega)$-modules. If $U \subset \operatorname{sing} M$

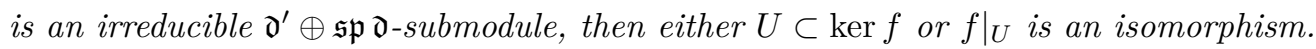


Proof. Follows from Schur's Lemma and the fact that $\left.f\right|_{U}$ is a homomorphism of $\mathcal{N}_{\mathcal{P}} / \mathcal{P}_{1^{-}}$ modules.

We would like to give a description of the action of $H(\mathfrak{d}, \chi, \omega)$ on singular vectors in terms of $\rho_{\text {sing. }}$. However, when using Proposition 2.1, it is better to express (2.37) in terms of Fourier coefficients of the form $x^{i} e^{-\chi} \otimes_{H} e$. In order to do so, we need the following result.

Proposition 6.3. If $v \in \operatorname{sing} V$, then the action of $H(\mathfrak{d}, \chi, \omega)$ on $v$ is given by

$$
\begin{aligned}
e * v & =\sum_{i, j=1}^{2 N}\left(\bar{\partial}_{i} \bar{\partial}_{j} \otimes 1\right) \otimes_{H} \rho_{\text {sing }}\left(f^{i j}\right) v+(1 \otimes 1) \otimes_{H} \rho_{\text {sing }}(c) v \\
& -\sum_{k=1}^{2 N}\left(\bar{\partial}_{k} \otimes 1\right) \otimes_{H}\left(\rho_{\text {sing }}\left(\partial^{k}+\operatorname{ad}^{\mathfrak{s p}} \partial^{k}\right) v-\partial^{k} v\right) .
\end{aligned}
$$

Proof. As $\mathcal{P}_{1}$ acts trivially on singular vectors, Remark 2.2 shows that the action of $e$ on a singular vector $v$ is given by the following expression

$$
\begin{aligned}
e * v & =\sum_{1 \leq i<j \leq 2 N}\left(\overline{S\left(\partial_{i} \partial_{j}\right)} \otimes 1\right) \otimes_{H}\left(x^{i} x^{j} e^{-\chi} \otimes_{H} e\right) \cdot v \\
& +\sum_{i=1}^{2 N}\left(\overline{S\left(\partial_{i}^{2}\right)} \otimes 1\right) \otimes_{H}\left(\frac{1}{2}\left(x^{i}\right)^{2} e^{-\chi} \otimes_{H} e\right) \cdot v \\
& +\sum_{k=1}^{2 N}\left(\overline{S\left(\partial_{k}\right)} \otimes 1\right) \otimes_{H}\left(x^{k} e^{-\chi} \otimes_{H} e\right) \cdot v \\
& +(\overline{S(1)} \otimes 1) \otimes_{H}\left(e^{-\chi} \otimes_{H} e\right) \cdot v .
\end{aligned}
$$

This rewrites as

$$
\begin{aligned}
e * v & =\sum_{1 \leq i<j \leq 2 N}\left(\bar{\partial}_{j} \bar{\partial}_{i} \otimes 1\right) \otimes_{H}\left(x^{i} x^{j} \otimes_{H} e\right) \cdot v \\
& +\frac{1}{2} \sum_{i=1}^{2 N}\left(\bar{\partial}_{i}^{2} \otimes 1\right) \otimes_{H}\left(\left(x^{i}\right)^{2} \otimes_{H} e\right) \cdot v \\
& -\sum_{k=1}^{2 N}\left(\bar{\partial}_{k} \otimes 1\right) \otimes_{H}\left(x^{k} e^{-\chi} \otimes_{H} e\right) \cdot v \\
& +(1 \otimes 1) \otimes_{H}\left(e^{-\chi} \otimes_{H} e\right) \cdot v
\end{aligned}
$$

where we used that $x^{i} x^{j} e^{-\chi}$ coincides with $x^{i} x^{j}$ up to higher degree terms. We may use Lemma 3.6 to express the third summand on the right-hand side as 


$$
\begin{aligned}
& \sum_{k=1}^{2 N}\left(\bar{\partial}_{k} \otimes 1\right) \otimes_{H}\left(x^{k} e^{-\chi} \otimes_{H} e\right) \cdot v \\
= & \sum_{k=1}^{2 N}\left(\bar{\partial}_{k} \otimes 1\right) \otimes_{H}\left(\rho_{\operatorname{sing}}\left(\partial^{k}+\operatorname{ad}^{\mathfrak{s p}} \partial^{k}\right) v-\partial^{k} v-\sum_{1 \leq i<j \leq 2 N} c_{i j}^{k} \rho_{\operatorname{sing}}\left(f^{i j}\right) v\right)
\end{aligned}
$$

Now we rewrite the first summand in the right-hand side using (6.1) and the fact that $2 \bar{\partial}_{j} \bar{\partial}_{i}=\left(\bar{\partial}_{i} \bar{\partial}_{j}+\bar{\partial}_{j} \bar{\partial}_{i}\right)-\left[\bar{\partial}_{i}, \bar{\partial}_{j}\right]$. Then the first two summands together yield

$$
\sum_{i, j=1}^{2 N}\left(\bar{\partial}_{i} \bar{\partial}_{j} \otimes 1\right) \otimes_{H}\left(\rho_{\operatorname{sing}}\left(f^{i j}\right) v\right)-\sum_{k=1}^{2 N}\left(\bar{\partial}_{k} \otimes 1\right) \otimes_{H} \sum_{1 \leq i<j \leq 2 N} c_{i j}^{k} \rho_{\operatorname{sing}}\left(f^{i j}\right) v .
$$

The two summations over $i<j$ then cancel each other, giving (6.4).

Corollary 6.1. Let $V$ be an $H(\mathfrak{d}, \chi, \omega)$-module, and $R$ be a $\mathfrak{d}^{\prime} \oplus \mathfrak{s p} \mathfrak{d}$-submodule of $\operatorname{sing} V$. Denote by $H R$ the $H$-submodule of $V$ generated by $R$. Then $H R$ is a $H(\mathfrak{d}, \chi, \omega)$ submodule of $V$. In particular, if $V$ is irreducible and $R$ is non-zero, then $V=H R$.

Proof. By (6.4), we have $H(\mathfrak{d}, \chi, \omega) * R \subset(H \otimes H) \otimes_{H} H R$. Then $H$-bilinearity implies that $H R$ is a $H(\mathfrak{d}, \chi, \omega)$-submodule of $V$.

\subsection{More tensor modules}

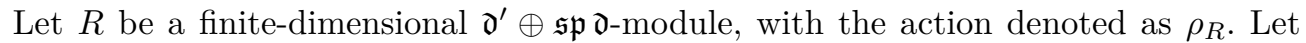
$V=H \otimes R$ be the free $H$-module generated by $R$, where $H$ acts by a left multiplication on the first factor. Mimicking (6.4), we define an action $(v \in R)$ :

$$
\begin{aligned}
e * v & =\sum_{i, j=1}^{2 N}\left(\bar{\partial}_{i} \bar{\partial}_{j} \otimes 1\right) \otimes_{H}\left(1 \otimes \rho_{R}\left(f^{i j}\right) v\right)+(1 \otimes 1) \otimes_{H}\left(1 \otimes \rho_{R}(c) v\right) \\
& -\sum_{k=1}^{2 N}\left(\bar{\partial}_{k} \otimes 1\right) \otimes_{H}\left(1 \otimes \rho_{R}\left(\partial^{k}+\operatorname{ad}^{\mathfrak{s p}} \partial^{k}\right) v-\partial^{k} \otimes v\right),
\end{aligned}
$$

and then extend it by $H$-bilinearity to a map $*: H(\mathfrak{d}, \chi, \omega) \otimes V \rightarrow(H \otimes H) \otimes_{H} V$.

Lemma 6.4. Let $R$ be a finite-dimensional $\mathfrak{d}^{\prime} \oplus \mathfrak{s p \mathfrak { d } - m o d u l e}$ with an action $\rho_{R}$. Then formula (6.5) defines a structure of an $H(\mathfrak{d}, \chi, \omega)$-module on $V=H \otimes R$. We have $\mathbf{k} \otimes R \subset \operatorname{sing} V$ and

$$
\rho_{\text {sing }}(A)(1 \otimes u)=1 \otimes \rho_{R}(A) u, \quad A \in \mathfrak{d}^{\prime} \oplus \mathfrak{s p \mathfrak { d }}, \quad u \in R .
$$

Proof. This can be showed by means of a lengthy but straightforward computation. Alternatively, the identification $\mathfrak{d}^{\prime} \oplus \mathfrak{s p} \mathfrak{d}=\mathcal{N}_{\mathcal{P}} / \mathcal{P}_{1}$ makes $R$ into a conformal $\mathcal{N}_{\mathcal{P}}$-module 
with a trivial $\mathcal{P}_{1}$-action. As $\widetilde{\mathcal{P}}=\mathfrak{d} \oplus \mathcal{N}_{\mathcal{P}}$ as vector spaces, the induced module $\operatorname{Ind}_{\mathcal{N}_{\mathcal{P}}}{ }^{\widetilde{\mathcal{P}}}$ is isomorphic to $U(\mathfrak{d}) \otimes R=H \otimes R$. The $H$-module structure is then given by multiplication on the first factor, whereas the $\mathcal{N}_{\mathcal{P}} / \mathcal{P}_{1}$-action on $\mathbf{k} \otimes R$ is as given in (6.6) by direct comparison of (6.4) with (6.5). Thus we have built a conformal $\widetilde{\mathcal{P}}$-module - i.e., an $H(\mathfrak{d}, \chi, \omega)$-module - structure on $H \otimes R$.

Vectors in $\mathbf{k} \otimes R$ will be referred to as constant singular vectors.

Definition 6.2. (i) Let $R$ be a finite-dimensional $\mathfrak{d}^{\prime} \oplus \mathfrak{s p} \mathfrak{d}$-module with an action $\rho_{R}$. Then the $H(\mathfrak{d}, \chi, \omega)$-module $H \otimes R$, with the action of $H(\mathfrak{d}, \chi, \omega)$ given by $(6.5)$, will be denoted as $\mathcal{V}(R)$.

(ii) Let $R=\Pi^{\prime} \otimes U$, where $\Pi^{\prime}$ is a finite-dimensional $\mathfrak{d}^{\prime}$-module and $U$ is a finitedimensional $\mathfrak{s p} \mathfrak{d}$-module. Then the module $\mathcal{V}(R)$ will also be denoted as $\mathcal{V}\left(\Pi^{\prime}, U\right)$.

(iii) As before, when $R=\Pi \otimes U$, where $\Pi$ is a finite-dimensional $\mathfrak{d}$-module and $U$ is a finite-dimensional $\mathfrak{s p d}$-module, we will abuse the notation and write $\mathcal{V}(R)=\mathcal{V}(\Pi, U):=$ $\mathcal{V}\left(\pi^{*} \Pi, U\right)$, where $\pi^{*} \Pi$ is the $\mathfrak{d}^{\prime}$-module obtained by lifting the $\mathfrak{d}$-action on $\Pi$ to $\mathfrak{d}^{\prime}$ via composing with the canonical projection $\pi: \mathfrak{d}^{\prime} \rightarrow \mathfrak{d}^{\prime} / \mathbf{k} c \simeq \mathfrak{d}$.

It is possible to translate Definitions 4.1 and 6.2 into one another.

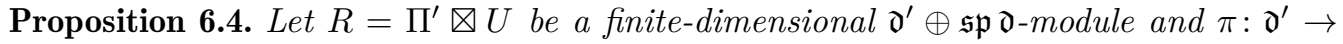
$\mathfrak{d}^{\prime} / \mathbf{k} c \simeq \mathfrak{d}$ denote the canonical projection. Then $\mathcal{V}\left(\Pi^{\prime}, U\right)=\mathcal{T}\left(\Pi_{\pi^{*} \phi}^{\prime}, U\right)$ and $\mathcal{T}\left(\Pi^{\prime}, U\right)=$ $\mathcal{V}\left(\Pi_{-\pi^{*} \phi}^{\prime}, U\right)$, where $\phi=-N \chi+\operatorname{trad}$ and $\pi^{*} \phi=\phi \circ \pi$ is the corresponding trace form on $\mathfrak{d}^{\prime}$.

Proof. The two claims are clearly equivalent, so it suffices to prove the latter. Take the definition of $\mathcal{T}\left(\Pi^{\prime}, U\right)$ given in (4.10), and rewrite the first summand in the right-hand side of (4.10) as follows:

$$
\begin{array}{r}
\sum_{k=1}^{2 N}\left(\bar{\partial}_{k} \otimes \partial^{k}\right) \otimes_{H} v=\sum_{k=1}^{2 N}\left(\bar{\partial}_{k} \otimes 1\right) \otimes_{H} \partial^{k} v-\sum_{k=1}^{2 N}\left(\bar{\partial}_{k}\left(\bar{\partial}^{k}+\chi\left(\partial^{k}\right)\right) \otimes 1\right) \otimes_{H} v \\
=\sum_{k=1}^{2 N}\left(\bar{\partial}_{k} \otimes 1\right) \otimes_{H} \partial^{k} v-\sum_{k=1}^{2 N}\left(\bar{\partial}_{k} \bar{\partial}^{k} \otimes 1\right) \otimes_{H} v-\sum_{k=1}^{2 N}\left(\bar{\partial}_{k} \otimes 1\right) \otimes_{H} \chi\left(\partial^{k}\right) v
\end{array}
$$

Using (3.21) and (3.4), we get:

$$
\sum_{k=1}^{2 N} \bar{\partial}_{k} \bar{\partial}^{k}=\bar{\rho}=-\sum_{k=1}^{2 N} \bar{\partial}_{k} \iota_{\rho} \omega\left(\partial^{k}\right),
$$

which together with (3.22) implies 


$$
\sum_{k=1}^{2 N}\left(\bar{\partial}_{k} \otimes \partial^{k}\right) \otimes_{H} v=\sum_{k=1}^{2 N}\left(\bar{\partial}_{k} \otimes 1\right) \otimes_{H} \partial^{k} v+\sum_{k=1}^{2 N}\left(\bar{\partial}_{k} \otimes 1\right) \otimes_{H} \phi\left(\partial^{k}\right) v
$$

Hence, (4.10) rewrites as

$$
\begin{aligned}
e * v & =\sum_{i, j=1}^{2 N}\left(\bar{\partial}_{i} \bar{\partial}_{j} \otimes 1\right) \otimes_{H}\left(1 \otimes f^{i j} \cdot v\right)+(1 \otimes 1) \otimes_{H}(1 \otimes c \cdot v) \\
& -\sum_{k=1}^{2 N}\left(\bar{\partial}_{k} \otimes 1\right) \otimes_{H}\left(1 \otimes\left(\partial^{k}+\operatorname{ad}^{\mathfrak{s p}} \partial^{k}\right) \cdot v-\phi\left(\partial^{k}\right) \otimes v-\partial^{k} \otimes v\right) .
\end{aligned}
$$

Comparing this to (6.5) completes the proof.

The following statements are analogous to those proved in [1-3].

Theorem 6.1. Let $V$ be an irreducible finite $H(\mathfrak{d}, \chi, \omega)$-module, and $R$ be an irreducible

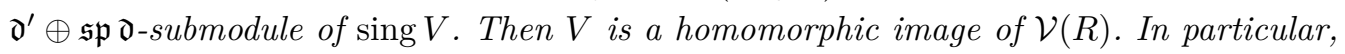
every irreducible finite $H(\mathfrak{d}, \chi, \omega)$-module is a quotient of a tensor module.

Proof. By (6.4) and (6.5) the canonical projection $\mathcal{V}(R)=H \otimes R \rightarrow H R$ is a homomorphism of $H(\mathfrak{d}, \chi, \omega)$-modules. However, $H R=V$ by Corollary 6.1.

We will now investigate how reducibility of a tensor module depends on existence of non-constant singular vectors. The picture is more involved than with primitive Lie pseudoalgebras of the other types.

Lemma 6.5. If $R$ is an irreducible representation of $\mathfrak{d}^{\prime} \oplus \mathfrak{s p} \mathfrak{d}$, then any non-zero proper $H(\mathfrak{d}, \chi, \omega)$-submodule $M$ of $\mathcal{V}(R)=H \otimes R$ satisfies $M \cap(\mathbf{k} \otimes R)=\{0\}$.

Proof. Both $M$ and $\mathbf{k} \otimes R$ are $\mathfrak{d}^{\prime} \oplus \mathfrak{s p} \mathfrak{d}$-stable, and the same is true of their intersection. The claim now follows from irreducibility of $R$.

Corollary 6.2. If $R$ is an irreducible $\mathfrak{d}^{\prime} \oplus \mathfrak{s p} \mathfrak{d}$-module and $\operatorname{sing} \mathcal{V}(R)=\mathbf{k} \otimes R$, then the $H(\mathfrak{d}, \chi, \omega)$-module $\mathcal{V}(R)$ is irreducible.

Proof. Assume there is a non-zero proper submodule $M$. Then $M$ must contain some non-zero singular vector. However, $M \cap \operatorname{sing} \mathcal{V}(R)=\{0\}$ by Lemma 6.5.

A converse to Corollary 6.2 only holds for those tensor modules $\mathcal{V}(R)$ in which the $\mathcal{P}$-action factors through $\mathcal{H} \simeq \mathcal{P} / \mathbf{k} c$. Recall that $\mathcal{P}$ is graded by the eigenspace decomposition of ad $\mathcal{E}$, which also provides a grading for $\widetilde{\mathcal{P}}$ as $\mathcal{E}$ commutes with elements $\widehat{\partial}$ 
$(\partial \in \mathfrak{d})$. If $\mathfrak{p}_{n}$ denotes the graded summand of eigenvalue $n$, then one has the semidirect sum decomposition of Lie algebras

$$
\mathcal{N}_{\mathcal{P}}=\mathfrak{d}^{\prime} \ltimes \prod_{j \geq 0} \mathfrak{p}_{j}
$$

and the decomposition of vector spaces

$$
\widetilde{\mathcal{P}}=\mathfrak{p}_{-1} \oplus \mathcal{N}_{\mathcal{P}}
$$

Notice that $\mathfrak{p}_{-1}$ is not a subalgebra, as the Lie bracket of suitably chosen elements yields a nonzero multiple of the central element $c=e^{-\chi} \in \mathfrak{p}_{-2}$, which may act non-trivially on $R$.

However, if the action of $c$ is trivial on $R$ then the $\mathcal{P}$-action factors through $\mathcal{H}$, and we may repeat the above argument with the Lie algebra $\widetilde{\mathcal{H}}$ so as to obtain $\widetilde{\mathcal{H}}=\mathfrak{h}_{-1} \oplus \mathcal{N}_{\mathcal{H}}$. Then $\mathfrak{h}_{-1}$ is a graded (abelian) Lie algebra and its universal enveloping algebra is a symmetric algebra generated in degree -1 . Then $\mathcal{V}(R)=\operatorname{Ind}_{\mathcal{N}_{\mathcal{H}}}^{\widetilde{\mathcal{H}}} R$ is isomorphic to $S\left(\mathfrak{h}_{-1}\right) \otimes R$, which can be endowed with a $-\mathbb{Z}_{+}$-grading by setting elements from $R$ to have degree 0 , and elements from $\mathfrak{h}_{-i}$ to have degree $-i$. A vector $v \in \mathcal{V}(R)$ is then homogeneous if it has a single graded component with respect to the above grading. Since $\mathcal{N}_{\mathcal{H}} / \mathcal{H}_{1}$ may be identified with the degree 0 part $\widehat{\mathfrak{d}} \oplus \mathfrak{h}_{0} \simeq \mathfrak{d} \oplus \mathfrak{s p} \mathfrak{d}$, its action on singular vectors will map homogeneous vectors to homogeneous vectors of the same degree, thus showing that homogeneous components of singular vectors are singular vectors.

The above argument, used in the case where $c \in \mathfrak{p}_{-2}$ acts non-trivially on $R$, shows that $\mathcal{V}(R)$ is only $-\mathbb{Z}_{+}$-filtered, but certainly not graded, as the central element, which has degree -2 , acts via a nonzero scalar multiplication so preserving the degree of vectors in the representation.

Remark 6.1. Let $V$ be an irreducible $H(\mathfrak{d}, \chi, \omega)$-module on which the $\mathcal{P}$-action factors through $\mathcal{H}$. As $\mathcal{E}$ stabilizes $\mathcal{H}_{1}$ and commutes with $\mathfrak{h}_{0}$, isotypic components of $\operatorname{sing} V$ are stabilized by the action of $\mathcal{E}$. In particular, if an isotypic component of $\operatorname{sing} V$ is a single $\mathfrak{d} \oplus \mathfrak{s p} \mathfrak{d}$-irreducible summand, then $\mathcal{E}$ acts as multiplication by a scalar, and this summand is homogeneous. We will later see that in the framework of tensor $H(\mathfrak{d}, \chi, \omega)$ modules there is a single exception to homogeneity of irreducible summands in $\operatorname{sing} V$, which happens when $\chi=0$.

Proposition 6.5. Let $R$ be an irreducible $\mathfrak{d}^{\prime} \oplus \mathfrak{s p} \mathfrak{d}$-module on which $c \in \mathfrak{d}^{\prime}$ acts trivially. Then every non-constant homogeneous singular vector in $\mathcal{V}(R)$ is contained in a non-zero proper submodule. In particular, $\mathcal{V}(R)$ is irreducible if and only if $\operatorname{sing} \mathcal{V}(R)=\mathbf{k} \otimes R$.

Proof. Every non-constant homogeneous singular vector $s$ is of negative degree $d<0$. Then $U(\widetilde{\mathcal{H}}) s=S\left(\mathfrak{h}_{-1}\right) s$ is a non-zero $H(\mathfrak{d}, \chi, \omega)$-submodule of $\mathcal{V}(R)$ lying in degrees $\leq d$, and intersects $R$ trivially, as $R$ lies in degree zero. 


\subsection{Filtration of tensor modules}

Tensor $H(\mathfrak{d}, \chi, \omega)$-modules possess a filtration induced by the canonical increasing filtration $\left\{\mathrm{F}^{n} H\right\}$. We may in fact define

$$
\mathrm{F}^{n} \mathcal{V}(R)=\mathrm{F}^{n} H \otimes R, \quad n=-1,0, \ldots
$$

As usual, $\mathrm{F}^{-1} \mathcal{V}(R)=\{0\}$ and $\mathrm{F}^{0} \mathcal{V}(R)=\mathbf{k} \otimes R$, and elements lying in $\mathrm{F}^{n} \mathcal{V}(R) \backslash$ $\mathrm{F}^{n-1} \mathcal{V}(R)$ are called vectors of degree $n$. Notice that vectors of degree 0 will be often referred to as constant vectors; they are all singular, by Lemma 6.4.

The associated graded space is defined accordingly, and we have isomorphisms of vector spaces

$$
\operatorname{gr}^{n} \mathcal{V}(R) \simeq S^{n} \mathfrak{d} \otimes R
$$

where $S^{n} \mathfrak{d}$ is the $n$-th symmetric power of the vector space $\mathfrak{d}$. Note that we can make $\mathfrak{d}$ into an $\mathfrak{s p} \mathfrak{d}$-module via the inclusion $\mathfrak{s p} \mathfrak{d} \subset \mathfrak{g l} \mathfrak{d}$. Then $\mathfrak{d}$ is an irreducible $\mathfrak{s p} \mathfrak{d}$-module. In a similar way $S^{n} \mathfrak{d}$ will be understood as an $\mathfrak{s p} \mathfrak{d}$-module.

Lemma 6.6. For every $p, n \geq 0$, we have:

$$
\begin{aligned}
\mathfrak{d} \cdot \mathrm{F}^{p} \mathcal{V}(R) & =\mathrm{F}^{p+1} \mathcal{V}(R), \\
\mathcal{N}_{\mathcal{P}} \cdot \mathrm{F}^{p} \mathcal{V}(R) & \subset \mathrm{F}^{p} \mathcal{V}(R), \\
\mathcal{P} \cdot \mathrm{F}^{p} \mathcal{V}(R) & \subset \mathrm{F}^{p+1} \mathcal{V}(R), \\
\mathcal{P}_{n} \cdot \mathrm{F}^{p} \mathcal{V}(R) & \subset \mathrm{F}^{p-n} \mathcal{V}(R) .
\end{aligned}
$$

Proof. The proof of (6.11)-(6.13) is the same as in the case of $W(\mathfrak{d})$ (see [2, Lemma 6.3]). Similarly, (6.14) follows using (6.13) and the fact that $\left[\mathfrak{d}, \mathcal{P}_{n}\right] \subset \mathcal{P}_{n-1},\left[\mathfrak{d}, \mathcal{P}_{1}\right] \subset$ $\mathcal{P}_{0} \subset \mathcal{N}_{\mathcal{P}}$.

As a consequence of Lemma 6.6 , we see that both $\mathcal{N}_{\mathcal{P}}$ and its quotient $\mathcal{N}_{\mathcal{P}} / \mathcal{P}_{1} \simeq$ $\mathfrak{d}^{\prime} \oplus \mathfrak{s p} \mathfrak{d}$ act on each space $\operatorname{gr}^{p} \mathcal{V}(R)$.

Lemma 6.7. The action of $\mathfrak{d}^{\prime}$ and $\mathcal{P}_{0} / \mathcal{P}_{1} \simeq \mathfrak{s p} \mathfrak{d}$ on the space $\operatorname{gr}^{p} \mathcal{V}(R) \simeq S^{p} \mathfrak{d} \otimes R$ satisfies

$$
\begin{aligned}
& \widehat{\partial} \cdot(f \otimes u)=f \otimes\left(\rho_{R}(\partial) u+\frac{p}{2} \chi(\partial) u\right), \\
& A \cdot(f \otimes u)=A f \otimes u+f \otimes \rho_{R}(A) u,
\end{aligned}
$$

for $A \in \mathfrak{s p \mathfrak { d }}, f \in S^{p} \mathfrak{d}, u \in R$.

Proof. One only needs to pay attention to the fact that, by (3.34), the action of $\widehat{\partial}$ on $\mathfrak{d}$ is given by scalar multiplication by $\chi(\partial) / 2$. 
Corollary 6.3. Let $\Pi^{\prime}, U$ be irreducible representations of $\mathfrak{d}^{\prime}, \mathfrak{s p} \mathfrak{d}$ respectively. Then there

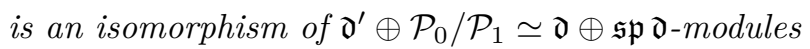

$$
\operatorname{gr}^{p} \mathcal{V}\left(\Pi^{\prime}, U\right) \simeq \Pi_{p \pi^{*} \chi / 2}^{\prime} \otimes\left(S^{p} \mathfrak{d} \otimes U\right)
$$

In particular, when the action of $\mathcal{P}$ factors through the quotient $\mathcal{H}$ and $\Pi^{\prime}=\pi^{*} \Pi$, one has

$$
\operatorname{gr}^{p} \mathcal{V}(\Pi, U) \simeq \Pi_{p \chi / 2} \otimes\left(S^{p} \mathfrak{d} \otimes U\right)
$$

whereas, when the action of $c \in \mathfrak{d}^{\prime}$ on $\Pi^{\prime}$ is non-trivial (whence $\chi$ vanishes), one has

$$
\operatorname{gr}^{p} \mathcal{V}\left(\Pi^{\prime}, U\right) \simeq \Pi^{\prime} \otimes\left(S^{p} \mathfrak{d} \otimes U\right)
$$

Proof. Follows immediately from Lemma 6.7.

Remark 6.2. Notice that when $\chi \neq 0$ one has

$$
\mathrm{F}^{p} \mathcal{V}(\Pi, U) \simeq \bigoplus_{i=0}^{p} \Pi_{i \chi / 2} \otimes\left(S^{i} \mathfrak{d} \otimes U\right)
$$

by complete reducibility of the $\mathfrak{s p}$ d-action, as each $\operatorname{gr}^{i} \mathcal{V}(\Pi, U), 0 \leq i \leq p$, sits in distinct isotypical components.

We will later use the following fact:

Lemma 6.8. The $H(\mathfrak{d}, \chi, \omega)$-module homomorphisms $\mathrm{d}_{\Pi}^{i}, 1 \leq i \leq N$, and $\mathrm{d}_{\Pi_{-\chi}}^{j}, N+1 \leq$ $j \leq 2 N$, showing up in each (twisted) conformally symplectic pseudo de Rham complex (5.36), map constant singular vectors to vectors of degree 1.

Proof. All such maps are induced by restriction or projection from the corresponding $W(\mathfrak{d})$-pseudo de Rham differentials, which satisfy the same property by [2, Lemma $5.3]$.

\section{Irreducibility of tensor modules}

We have seen that all irreducible finite representations of $H(\mathfrak{d}, \chi, \omega)$ arise as quotients of some special $H(\mathfrak{d}, \chi, \omega)$-modules called tensor modules. A classification of irreducible $H(\mathfrak{d}, \chi, \omega)$-modules will then follow from a description of irreducible quotients of tensor modules. 


\subsection{Coefficients of elements and submodules}

Throughout this section, $R$ will be an irreducible $\mathfrak{d}^{\prime} \oplus \mathfrak{s p} \mathfrak{d}$-module, with the action denoted by $\rho_{R}$, and $\mathcal{V}(R)$ the corresponding tensor module introduced in Definition 6.2. First, let us rewrite the expression (6.5) for the action of $e$ on singular vectors in a tensor module in a different fashion. We introduce the notation

$$
\psi(u)=\sum_{i, j=1}^{2 N} \partial_{i} \partial_{j} \otimes \rho_{R}\left(f^{i j}\right) u
$$

Then we have for $u \in R$ :

$$
\begin{aligned}
& e *(1 \otimes u)=(1 \otimes 1) \otimes_{H}\left(\psi(u)-\sum_{k=1}^{2 N} \partial_{k} \otimes \rho_{R}\left(\partial^{k}\right) u\right) \\
& \quad+\text { terms in }(1 \otimes H) \otimes_{H}\left(\mathfrak{d} \otimes\left(\mathbf{k}+\rho_{R}(\mathfrak{s p} \mathfrak{d})\right) u+\mathbf{k} \otimes\left(\mathbf{k}+\rho_{R}\left(\mathfrak{s p} \mathfrak{d}+\mathfrak{d}^{\prime}\right)\right) u\right)
\end{aligned}
$$

On the other hand, if $v \in \operatorname{sing} \mathcal{V}(R)$, then (6.4) right-straightens to

$$
\begin{aligned}
e * v & =\sum_{i, j=1}^{2 N}\left(1 \otimes \partial_{i} \partial_{j}\right) \otimes_{H} \rho_{\text {sing }}\left(f^{i j}\right) v \\
& \left.-\sum_{i=1}^{2 N}\left(1 \otimes \partial_{i}\right) \otimes_{H}\left(\rho_{\text {sing }}\left(\partial^{i}+\operatorname{ad}^{\mathfrak{s p}} \partial^{i}\right) v-\partial^{i} v+2 \sum_{j=1}^{2 N} \bar{\partial}_{j} \rho_{\operatorname{sing}}\left(f^{i j}\right) v\right)\right) \\
& + \text { terms in }(1 \otimes 1) \otimes_{H} \mathcal{V}(R) .
\end{aligned}
$$

Lemma 7.1. If $v=\sum_{I} \partial^{(I)} \otimes v_{I}$, then

$$
\begin{aligned}
e * v & =\sum_{I}\left(1 \otimes \partial^{(I)}\right) \otimes_{H} \psi\left(v_{I}\right) \\
& + \text { terms in }\left(1 \otimes \partial^{(I)} H\right) \otimes_{H}\left(\mathrm{~F}^{1} H \otimes\left(\mathbf{k}+\rho_{R}\left(\mathfrak{s p} \mathfrak{d}+\mathfrak{d}^{\prime}\right)\right) v_{I}\right) .
\end{aligned}
$$

In particular, the coefficient multiplying $1 \otimes \partial^{(I)}$ equals $\psi\left(v_{I}\right)$ modulo $\mathrm{F}^{1} \mathcal{V}(R)$.

Proof. This follows from (7.2).

Recall that every element $v$ of $\mathcal{V}(R)$ can be uniquely expressed in the form

$$
v=\sum_{I} \partial^{(I)} \otimes v_{I} .
$$

Definition 7.1. The non-zero elements $v_{I}$ in the above expression are called coefficients of $v \in \mathcal{V}(R)$. For a submodule $M \subset \mathcal{V}(R)$, we denote by coeff $M$ the subspace of $R$ spanned over $\mathbf{k}$ by all coefficients of elements from $M$. 
Lemma 7.2. Let $R$ be an irreducible $\mathfrak{d}^{\prime} \oplus \mathfrak{s p \mathfrak { d } - m o d u l e . ~ T h e n , ~ f o r ~ a n y ~ n o n - z e r o ~ p r o p e r ~}$ $H(\mathfrak{d}, \chi, \omega)$-submodule $M$ of $\mathcal{V}(R)$, we have coeff $M=R$.

Proof. Pick a non-zero element $v=\sum_{I} \partial^{(I)} \otimes v_{I}$ contained in $M$. Then Lemma 7.1 shows that $M$ contains an element congruent to $\psi\left(v_{I}\right)$ modulo $\mathrm{F}^{1} \mathcal{V}(R)$, thus coefficients of $\psi\left(v_{I}\right)$ all lie in coeff $M$ for all $I$. This proves that $\rho_{R}(\mathfrak{s p} \mathfrak{d})($ coeff $M) \subset$ coeff $M$. Similarly, one can write

$$
\begin{aligned}
& e * v=\sum_{I}\left(1 \otimes \partial^{(I)}\right) \otimes_{H}\left(\psi\left(v_{I}\right)-\sum_{k} \partial_{k} \otimes \rho_{R}\left(\partial^{k}\right) v_{I}\right) \\
& \left.\quad+\text { terms in }\left(1 \otimes \partial^{(I)} H\right) \otimes_{H}\left(\mathfrak{d} \otimes \rho_{R}(\mathfrak{s p} \mathfrak{d}+\mathbf{k})\right) v_{I}+\mathbf{k} \otimes\left(\mathbf{k}+\rho_{R}\left(\mathfrak{s p} \mathfrak{d}+\mathfrak{d}^{\prime}\right)\right) v_{I}\right),
\end{aligned}
$$

showing that $\rho_{R}\left(\partial^{k}\right) v_{I} \in \operatorname{coeff} M$ for all $I, k=1, \ldots, 2 N$. Thus, $\rho_{R}\left(\mathfrak{d}^{\prime}\right)($ coeff $M) \subset$ coeff $M$, as the central element $c$ acts via multiplication by a scalar. Then coeff $M$ is a non-zero $\mathfrak{d}^{\prime} \oplus \mathfrak{s p} \mathfrak{d}$-submodule of $R$. Irreducibility of $R$ now gives coeff $M=R$ as soon as $M \neq 0$.

Corollary 7.1. Let $M$ be a non-zero proper $H(\mathfrak{d}, \chi, \omega)$-submodule of $\mathcal{V}(R)$. Then for every $u \in R$ there is some element in $M$ coinciding with $\psi(u)$ modulo $\mathrm{F}^{1} \mathcal{V}(R)$.

Proof. As coeff $M=R$, it is enough to prove the statement for coefficients of elements lying in $M$. In order to do so, pick $v \in M$ and proceed as in Lemma 7.1. As $M$ is an $H(\mathfrak{d}, \chi, \omega)$-submodule of $\mathcal{V}(R)$, the coefficient multiplying $1 \otimes \partial^{(I)}$ still lies in $M$, and equals $\psi\left(v_{I}\right)$ modulo $\mathrm{F}^{1} \mathcal{V}(R)$.

The following lemma will allow us to derive an irreducibility criterion for tensor modules over $H(\mathfrak{d}, \chi, \omega)$. We state in a slightly different way as compared to [3], which will prove more versatile in the next section.

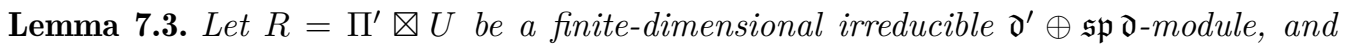
assume that $\psi(u)$ satisfies $e * \psi(u) \in\left(\mathrm{F}^{3} H \otimes \mathbf{k}\right) \otimes_{H} \mathcal{V}(R)$ for all u lying in a non-zero

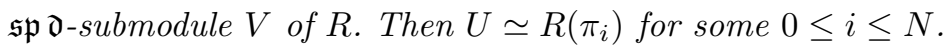

Proof. We proceed as in [3, Section 7.2]. The coefficient multiplying $1 \otimes \partial_{a} \partial_{b} \partial_{c} \partial_{d}$ in the right-straightened expression for $e * \psi(u)$ equals

$$
\sum f^{a b} f^{c d}(u)
$$

where the sum is over all permutations of $a, b, c, d$, and this must vanish for all $u \in V$, as $e * \psi(u) \in\left(\mathrm{F}^{3} H \otimes \mathbf{k}\right) \otimes_{H} \mathcal{V}(R)=\left(\mathbf{k} \otimes \mathrm{F}^{3} H\right) \otimes_{H} \mathcal{V}(R)$. As the $\mathfrak{s p} \mathfrak{d}$-module $R$ is $U$-isotypical, so is $V$, and expression (7.5) must vanish for every $u \in U$. However, as in [3, Theorem 7.1], these identities characterize $R\left(\pi_{i}\right), 0 \leq i \leq N$, among irreducible $\mathfrak{s p} \mathfrak{d}$-modules. 
Then we have:

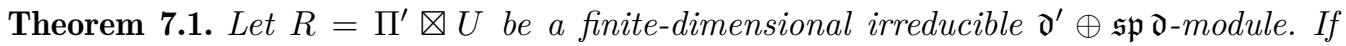
$U$ is neither trivial nor isomorphic to one of the fundamental representations $R\left(\pi_{i}\right)$, $i=1, \ldots, N$, then the tensor $H(\mathfrak{d}, \chi, \omega)$-module $\mathcal{V}(R)=\mathcal{V}\left(\Pi^{\prime}, U\right)$ is irreducible.

Proof. The proof follows the same lines as in [3, Section 7.2]. If $\mathcal{V}(R)$ is reducible, then by Corollary 7.1, there exists a non-trivial submodule $M \subset \mathcal{V}(R)$ containing an element $m(u)$ coinciding with $\psi(u)$ modulo $\mathrm{F}^{1} \mathcal{V}(R)$ for every $u \in R$. We know that

$$
\begin{aligned}
e * \mathrm{~F}^{1} \mathcal{V}(R) & \subset\left(\mathrm{F}^{2} H \otimes \mathrm{F}^{1} H\right) \otimes_{H} \mathcal{V}(R) \\
& \subset \mathrm{F}^{3}(H \otimes H) \otimes_{H} \mathcal{V}(R)=\left(\mathrm{F}^{3} H \otimes \mathbf{k}\right) \otimes_{H} \mathcal{V}(R),
\end{aligned}
$$

and $e * m(u) \in\left(\mathrm{F}^{4} H \otimes \mathbf{k}\right) \otimes_{H} M$ as $v \in M$. This shows that the coefficient multiplying $1 \otimes \partial_{a} \partial_{b} \partial_{c} \partial_{d}$ in the right-straightened expression for $e * \psi(u)$, which is given in (7.5), lies in $M \cap \mathrm{F}^{0} \mathcal{V}(R)=\{0\}$. Then we may apply Lemma 7.3 and conclude the proof of the theorem.

Notice that the converse certainly holds if the $\mathcal{P}$-action factors through $\mathcal{H}$ : in this case, if $U$ is either trivial or a fundamental representation, then $\mathcal{V}(\Pi, U)=\mathcal{V}\left(\pi^{*} \Pi, U\right)=$ $\mathcal{V}\left(\Pi^{\prime}, U\right)$ appears in a twisted conformally symplectic pseudo de Rham complex (see Theorem 5.1) and it is reducible, as the image or the kernel of each differential provides a non-trivial submodule.

Our final goal is to extend this fact to tensor modules in which $c$ acts non-trivially and to provide a complete classification of submodules. All this will be accomplished in next section by providing a description of all singular vectors.

\section{Computation of singular vectors}

In this section, we describe all singular vectors contained in a tensor $H(\mathfrak{d}, \chi, \omega)$-module $\mathcal{V}(R)=\mathcal{V}\left(\Pi^{\prime}, U\right)$, where $R=\Pi^{\prime} \otimes U$ is a finite-dimensional irreducible $\mathfrak{d}^{\prime} \oplus \mathfrak{s p} \mathfrak{d}$-module. As the case $U \simeq \mathbf{k}$ is of a somewhat different flavor, we treat it first; then we compute singular vectors of $\mathcal{V}\left(\Pi^{\prime}, R\left(\pi_{i}\right)\right)$ for $1 \leq i \leq N$. Finally, we show that, for any non-trivial irreducible $\mathfrak{s p} \mathfrak{d}$-module $U$, the existence of non-constant singular vectors in $\mathcal{V}\left(\Pi^{\prime}, U\right)$ forces $U$ to be isomorphic to some $R\left(\pi_{i}\right)$ with $1 \leq i \leq N$, thus completing the classification of singular vectors.

\subsection{Singular vectors in $\mathcal{V}\left(\Pi^{\prime}, \mathbf{k}\right)$}

Here we find all singular vectors in $\mathcal{V}\left(\Pi^{\prime}, \mathbf{k}\right)$.

Lemma 8.1. A vector in $\mathcal{V}\left(\Pi^{\prime}, \mathbf{k}\right)$ is singular if and only if it lies in $\mathrm{F}^{1} \mathcal{V}\left(\Pi^{\prime}, \mathbf{k}\right)$. 
Proof. Let us first prove $\operatorname{sing} \mathcal{V}(\Pi, \mathbf{k}) \subset \mathrm{F}^{1} \mathcal{V}\left(\Pi^{\prime}, \mathbf{k}\right)$. The $\mathfrak{s p} \mathfrak{d}$-action is trivial, so (6.5) can be rewritten as

$$
\begin{aligned}
e *(1 \otimes u) & =\sum_{k=1}^{2 N}\left(\bar{\partial}_{k} \otimes 1\right) \otimes_{H}\left(\partial^{k} \otimes u-1 \otimes \rho_{R}\left(\partial^{k}\right) u\right) \\
& +(1 \otimes 1) \otimes_{H}\left(1 \otimes \rho_{R}(c) u\right), \quad u \in R=\Pi^{\prime} \otimes \mathbf{k}
\end{aligned}
$$

This (or (7.3)) implies that

$$
\begin{aligned}
e *(1 \otimes u) & =-\sum_{k=1}^{2 N}\left(1 \otimes \partial_{k}\right) \otimes_{H}\left(\partial^{k} \otimes u-1 \otimes \rho_{R}\left(\partial^{k}\right) u\right) \\
& + \text { terms in }(1 \otimes 1) \otimes_{H} \mathcal{V}\left(\Pi^{\prime}, \mathbf{k}\right)
\end{aligned}
$$

Let now $v=\sum_{I} \partial^{(I)} \otimes v_{I}$ be a singular vector in $\mathcal{V}\left(\Pi^{\prime}, \mathbf{k}\right)$, and assume there is some $I$ with $|I|>1$ such that $v_{I} \neq 0$. If $n$ is the maximal value of $|I|$ for such $I$, choose among all $I=\left(i_{1}, \ldots, i_{2 N}\right)$ with $|I|=n$, one with the highest value of $i_{1}$. Then, by (8.2), the coefficient multiplying $1 \otimes \partial_{1} \partial^{(I)}$ in $e * v$ equals $-\partial^{1} \otimes v_{I}+1 \otimes \rho_{R}\left(\partial^{1}\right) v_{I}$. As $v$ is singular, this must vanish if $|I|>1$ by Lemma 6.1 , a contradiction with $v_{I} \neq 0$.

The reverse inclusion $\operatorname{sing} \mathcal{V}\left(\Pi^{\prime}, \mathbf{k}\right) \supset \mathrm{F}^{1} \mathcal{V}\left(\Pi^{\prime}, \mathbf{k}\right)$ is now easy. If $v \in \mathrm{F}^{1} \mathcal{V}\left(\Pi^{\prime}, \mathbf{k}\right)$, plugging it into (8.1) and left-straightening the resulting expression only yields terms in $\left(\mathrm{F}^{2} H \otimes \mathbf{k}\right) \otimes_{H} \mathcal{V}\left(\Pi^{\prime}, \mathbf{k}\right)$ and we are again done by Lemma 6.1.

Proposition 8.1. For an irreducible $\mathfrak{d}^{\prime}$-module $\Pi^{\prime}$, the $H(\mathfrak{d}, \chi, \omega)$-module $\mathcal{V}\left(\Pi^{\prime}, \mathbf{k}\right)$ is reducible if and only if the action of $c \in \mathfrak{d}^{\prime}$ on $\Pi^{\prime}$ is trivial.

Proof. Let $M$ be a non-zero proper submodule of $\mathcal{V}(R)=\mathcal{V}\left(\Pi^{\prime}, \mathbf{k}\right)$. We know that $M$ must contain non-constant singular vectors and that $\operatorname{gr}^{1} \mathcal{V}\left(\Pi^{\prime}, \mathbf{k}\right) \simeq \Pi_{\pi^{*} \chi / 2}^{\prime} \otimes \mathfrak{d}$ is $\mathfrak{d}^{\prime} \oplus \mathfrak{s p} \mathfrak{d}$ irreducible. Thus, for every choice of $\partial \in \mathfrak{d}$ and $u \in R$, there is an element in $M$ whose degree one term is $\partial \otimes u$.

Consequently, as $M \subset \mathcal{V}\left(\Pi^{\prime}, \mathbf{k}\right)$ is an $H$-submodule, $M$ must be of finite codimension in $\mathcal{V}\left(\Pi^{\prime}, \mathbf{k}\right)$; hence, $\mathcal{V}\left(\Pi^{\prime}, \mathbf{k}\right) / M$ is a torsion $H$-module. Since the action of a pseudoalgebra on torsion elements is trivial, we obtain $e * \mathcal{V}\left(\Pi^{\prime}, \mathbf{k}\right)=e * M \subset(H \otimes H) \otimes_{H} M$. Direct inspection of (8.1) now shows that $1 \otimes \rho_{R}(c) u$ belongs to $M$ for all $u \in R$. Then, if the action of $c$ is non-trivial, $M$ must contain non-zero constant vectors, which contradicts Lemma 6.5.

If instead the action of $c$ is trivial, then vectors $\partial \otimes u-1 \otimes \rho_{R}(\partial) u$, where $\partial \in \mathfrak{d}$, $u \in R$, all belong to $M$, and they indeed span over $H$ a proper $H(\mathfrak{d}, \chi, \omega)$-submodule of $\mathcal{V}\left(\Pi^{\prime}, \mathbf{k}\right)$.

Remark 8.1. Proposition 8.1 shows, by providing a counterexample, that requiring $c \in \mathfrak{d}^{\prime}$ to act trivially on $R$ in Proposition 6.5 is a mandatory hypothesis. 


\subsection{Non-trivial submodules of $\mathcal{V}\left(\Pi^{\prime}, \mathbf{k}\right)$}

We are only interested in the case where the action of $c \in \mathfrak{d}^{\prime}$ on $\Pi^{\prime}$ is trivial, as we have seen that $\mathcal{V}\left(\Pi^{\prime}, \mathbf{k}\right)$ is otherwise irreducible by Proposition 8.1 above. We thus focus on tensor modules $\mathcal{V}(\Pi, \mathbf{k}):=\mathcal{V}\left(\pi^{*} \Pi, \mathbf{k}\right)=\mathcal{V}\left(\Pi^{\prime}, \mathbf{k}\right)$, where $\Pi$ is a $\mathfrak{d}$-module, see Definition 6.2(iii).

First of all, observe that by (5.34) and Proposition 6.4, we have:

$$
\begin{aligned}
\Omega_{\Pi}^{2 N}(\mathfrak{d})=J_{\Pi}^{2 N}(\mathfrak{d}) & \simeq \mathcal{T}\left(\Pi_{-N \chi}, \mathbf{k}\right)=\mathcal{V}\left(\Pi_{-N \chi-\phi}, \mathbf{k}\right) \\
\Omega_{\Pi}^{2 N-1}(\mathfrak{d})=J_{\Pi}^{2 N-1}(\mathfrak{d}) & \simeq \mathcal{T}\left(\Pi_{-(2 N-1) \chi / 2}, R\left(\pi_{1}\right)\right)=\mathcal{V}\left(\Pi_{-(2 N-1) \chi / 2-\phi}, R\left(\pi_{1}\right)\right)
\end{aligned}
$$

Hence

$$
\begin{aligned}
\mathcal{V}(\Pi, \mathbf{k}) & \simeq J_{\Pi_{N \chi+\phi}}^{2 N}(\mathfrak{d})=\Omega_{\Pi_{N \chi+\phi}}^{2 N}(\mathfrak{d}), \\
\mathcal{V}\left(\Pi_{\chi / 2}, R\left(\pi_{1}\right)\right) & \simeq J_{\Pi_{N \chi+\phi}}^{2 N-1}(\mathfrak{d})=\Omega_{\Pi_{N \chi+\phi}-1}^{2 N-1}(\mathfrak{d}) .
\end{aligned}
$$

Recall also (cf. [2, Proposition 5.2 and Lemma 6.12]) that the image of the de Rham differential $\mathrm{d}_{\Pi_{N \chi+\phi}}: \Omega_{\Pi_{N \chi+\phi}}^{2 N-1}(\mathfrak{d}) \rightarrow \Omega_{\Pi_{N \chi+\phi}}^{2 N}(\mathfrak{d})$ is a maximal $H$-submodule which has finite codimension as a $\mathbf{k}$-vector subspace. This means that the image of the map

$$
\mathrm{d}_{\Pi_{\phi+N \chi}}^{2 N}: \mathcal{V}\left(\Pi_{\chi / 2}, R\left(\pi_{1}\right)\right) \rightarrow \mathcal{V}(\Pi, \mathbf{k})
$$

is a maximal $H(\mathfrak{d}, \chi, \omega)$-submodule of $\mathcal{V}(\Pi, \mathbf{k})$ of finite $\mathbf{k}$-codimension. Notice that $\mathrm{d}_{\Pi_{\phi+N \chi}}^{2 N} \mathrm{~F}^{0} \mathcal{V}\left(\Pi_{\chi / 2}, R\left(\pi_{1}\right)\right)$ is a $\mathfrak{d} \oplus \mathfrak{s p} \mathfrak{d}$-submodule of $\operatorname{sing} \mathcal{V}(\Pi, \mathbf{k})$ which has a non-zero projection of $\operatorname{gr}^{1} \mathcal{V}(\Pi, \mathbf{k}) \simeq \Pi_{\chi / 2} \otimes R\left(\pi_{1}\right)$, so that it coincides with the $(\mathfrak{d} \oplus \mathfrak{s p} \mathfrak{d})$-stable complement of $\mathrm{F}^{0} \mathcal{V}(\Pi, \mathbf{k})$ in $\operatorname{sing} \mathcal{V}(\Pi, \mathbf{k})$, which is uniquely determined as it coincides with the $R\left(\pi_{1}\right)$-isotypical component of $\mathrm{F}^{1} \mathcal{V}(\Pi, \mathbf{k})$.

Proposition 8.2. The unique non-zero proper $H(\mathfrak{d}, \chi, \omega)$-submodule of $\mathcal{V}(\Pi, \mathbf{k})$ is $\operatorname{Im} \mathrm{d}_{\Pi_{\phi+N \chi}}^{2 N}$.

Proof. We have proved in Lemma 8.1 that

$$
\operatorname{sing} \mathcal{V}(\Pi, \mathbf{k})=\mathrm{F}^{1} \mathcal{V}(\Pi, \mathbf{k}) \simeq \mathrm{F}^{0} \mathcal{V}(\Pi, \mathbf{k}) \oplus \operatorname{gr}^{1} \mathcal{V}(\Pi, \mathbf{k})
$$

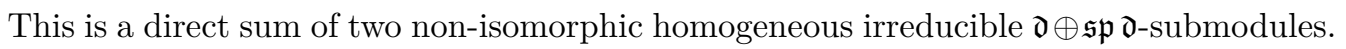
If $M \subset \mathcal{V}(\Pi, \mathbf{k})$ is a non-zero proper submodule, then it must contain non-zero homogeneous singular vectors, which cannot be constant by Lemma 6.5. Thus $M$ contains all of $\mathrm{d}_{\Pi_{\phi+N \chi}}^{2 N} \mathrm{~F}^{0} \mathcal{V}\left(\Pi_{\chi / 2}, R\left(\pi_{1}\right)\right)$, which spans $\operatorname{Im}_{\Pi_{\phi+N \chi}}^{2 N}$ over $H$. We see that $M$ contains the maximal submodule $\operatorname{Im} \mathrm{d}_{\Pi_{\phi+N \chi}}^{2 N}$, hence it coincides with it. 


\subsection{Singular vectors in $\mathcal{V}\left(\Pi^{\prime}, U\right)$ for a non-trivial $U$}

We now move to the case when the irreducible $\mathfrak{s p} \mathfrak{d}$-module $U$ is non-trivial, i.e., $U \neq \mathbf{k}$. We start with the following observation:

Proposition 8.3. Let $v \in \mathcal{V}\left(\Pi^{\prime}, U\right)$ be a singular vector, where $U$ is a non-trivial irreducible $\mathfrak{s p} \mathfrak{d}$-module. Then $v$ is of degree at most 2 .

Proof. Write $v=\sum_{I} \partial^{(I)} \otimes v_{I}$. Then Lemma 7.1, together with (7.3), shows that $\psi\left(v_{I}\right)=$ 0 whenever $|I| \geq 2$. As the $\mathfrak{s p} \mathfrak{d}$-action on $R=\Pi^{\prime} \otimes U$ is non-trivial, from $\psi\left(v_{I}\right)=0$ follows $v_{I}=0$.

Through the rest of this section, we will separately classify singular vectors of degree 1 and 2 in tensor modules of the form $\mathcal{V}\left(\Pi^{\prime}, R\left(\pi_{n}\right)\right)$ for $n=1, \ldots, N$. Then we will prove that if $U \not R R\left(\pi_{n}\right)$ for some $n=0, \ldots, N$, then $\mathcal{V}\left(\Pi^{\prime}, U\right)$ does not contain non-constant singular vectors, thus completing the classification.

\subsection{Singular vectors of degree one in $\mathcal{V}\left(\Pi^{\prime}, R\left(\pi_{n}\right)\right), 1 \leq n \leq N$}

We will proceed as follows: we will first treat the case $\mathcal{V}\left(\Pi^{\prime}, R\left(\pi_{n}\right)\right)$, where the action of $c \in \mathfrak{d}^{\prime}$ is trivial; then we will show that the description of singular vectors of degree 1 stays unchanged when the action of $c$ is via multiplication by a non-zero scalar.

\subsubsection{Singular vectors of degree one, graded case}

Our setting is the following: $\Pi$ and $U$ are irreducible representations of $\mathfrak{d}$ and $\mathfrak{s p} \mathfrak{d}$ respectively, $R=\pi^{*} \Pi \otimes U$ is a $\mathfrak{d}^{\prime} \oplus \mathfrak{s p} \mathfrak{d}$-module, and $V=\mathcal{V}(R)=\mathcal{V}(\Pi, U)$ is a tensor $H(\mathfrak{d}, \chi, \omega)$-module, as in Definition 6.2(iii). Furthermore, $U=R\left(\pi_{n}\right)$ for some $1 \leq n \leq N$. We assume that $V$ is not irreducible, and $M \subset V$ is a non-zero proper submodule. We first look for singular vectors of degree 1, i.e., of the form

$$
v=\sum_{i=1}^{2 N} \partial_{i} \otimes v^{i}+1 \otimes v^{0}, \quad v^{i} \in R
$$

which are contained in $M$.

Lemma 8.2. A non-zero singular vector of degree 1 , as in (8.3), contained in $M \subset V$ is uniquely determined by its homogeneous degree 1 part $\sum \partial_{i} \otimes v^{i}$.

Proof. If $v$ and $v^{\prime}$ are two such vectors agreeing in degree 1 , then $v-v^{\prime}$ is a singular vector contained in $M \cap(\mathbf{k} \otimes R)=\{0\}$ (see Lemma 6.5). 
Proposition 8.4. Let $v, v^{\prime}$ be non-zero singular vectors of degree 1 contained in (possibly distinct) non-zero proper submodules $M, M^{\prime}$ of a tensor module $\mathcal{V}(R)=\mathcal{V}\left(\Pi, R\left(\pi_{n}\right)\right.$ ) as above. If $v=v^{\prime} \bmod \mathrm{F}^{0} \mathcal{V}(R)$, then $v=v^{\prime}$.

Proof. We already know that each element in the irreducible $\mathfrak{d} \oplus \mathfrak{s p} \mathfrak{d}$-submodule $\mathrm{F}^{0} \mathcal{V}(R) \simeq \Pi \otimes R\left(\pi_{n}\right)$ is a singular vector. Moreover, $\operatorname{gr}^{1} \mathcal{V}(R)$ decomposes in the direct sum of (at most) three irreducible components isomorphic to $\Pi_{\chi / 2} \otimes R\left(\pi_{n-1}\right)$, $\Pi_{\chi / 2} \otimes R\left(\pi_{n+1}\right)$, and $\Pi_{\chi / 2} \otimes R\left(\pi_{n}+\pi_{1}\right)$, respectively. As no such representation, viewed as an $\mathfrak{s p} \mathfrak{d}$-module, has a non-zero $R\left(\pi_{n}\right)$-isotypical component, $\operatorname{gr}^{1} \mathcal{V}(R)$ splits off canonically and uniquely as a (homogeneous) complement of $\mathrm{F}^{0} \mathcal{V}(R)$ in $\mathrm{F}^{1} \mathcal{V}(R)$. If a singular vector $v \in \mathrm{F}^{1} \mathcal{V}(R)$ has a non-zero projection to $\operatorname{gr}^{1} \mathcal{V}(R)$ and is not contained in this canonical complement, then each $H(\mathfrak{d}, \chi, \omega)$-submodule of $\mathcal{V}(R)$ containing $v$ will also contain constant singular vectors, and will fail to be proper.

Recall that, by (5.33), (5.34) and Proposition 6.4, there are $H(\mathfrak{d}, \chi, \omega)$-module isomorphisms:

$$
\begin{aligned}
\Omega_{\Pi}^{n}(\mathfrak{d}) / I_{\Pi}^{n}(\mathfrak{d}) & \simeq \mathcal{T}\left(\Pi_{-n \chi / 2}, R\left(\pi_{n}\right)\right)=\mathcal{V}\left(\Pi_{-\phi-n \chi / 2}, R\left(\pi_{n}\right)\right) \\
J_{\Pi}^{2 N-n}(\mathfrak{d}) & \simeq \mathcal{T}\left(\Pi_{-(2 N-n) \chi / 2}, R\left(\pi_{n}\right)\right)=\mathcal{V}\left(\Pi_{-\phi-(2 N-n) \chi / 2}, R\left(\pi_{n}\right)\right)
\end{aligned}
$$

Hence

$$
\mathcal{V}\left(\Pi, R\left(\pi_{n}\right)\right) \simeq \Omega_{\Pi_{\phi+n \chi / 2}}^{n}(\mathfrak{d}) / I_{\Pi_{\phi+n \chi / 2}}^{n}(\mathfrak{d}) \simeq J_{\Pi_{\phi+(2 N-n) \chi / 2}}^{2 N-n}(\mathfrak{d})
$$

The conformally symplectic pseudo de Rham differentials

$$
\begin{aligned}
\mathrm{d}_{\Pi_{\phi+n \chi / 2}}^{n}: & \Omega_{\Pi_{\phi+n \chi / 2}}^{n-1}(\mathfrak{d}) / I_{\Pi_{\phi+n \chi / 2}}^{n-1}(\mathfrak{d}) \rightarrow \Omega_{\Pi_{\phi+n \chi / 2}}^{n}(\mathfrak{d}) / I_{\Pi_{\phi+n \chi / 2}}^{n}(\mathfrak{d}), \\
\mathrm{d}_{\Pi_{\phi+(2 N-n) \chi / 2}}^{2 N-n}: & : J_{\Pi_{\phi+(2 N-n) \chi / 2}}^{2 N-n-1}(\mathfrak{d}) \rightarrow J_{\Pi_{\phi+(2 N-n) \chi / 2}}^{2 N-n}(\mathfrak{d}),
\end{aligned}
$$

that we denote as in (5.36), (5.37), then correspond to tensor $H(\mathfrak{d}, \chi, \omega)$-module homomorphisms

$$
\begin{array}{rlrl}
\mathrm{d}_{\Pi_{\phi+n \chi / 2}}^{n}: \mathcal{V}\left(\Pi_{\chi / 2}, R\left(\pi_{n-1}\right)\right) & \rightarrow \mathcal{V}\left(\Pi, R\left(\pi_{n}\right)\right), & n=1, \ldots, N, \\
\mathrm{~d}_{\Pi_{\phi+(2 N-n) \chi / 2}^{2 N-n}}^{2 N-n}\left(\Pi_{\chi / 2}, R\left(\pi_{n+1}\right)\right) \rightarrow \mathcal{V}\left(\Pi, R\left(\pi_{n}\right)\right), & n=0, \ldots, N-1 .
\end{array}
$$

We similarly obtain an $H(\mathfrak{d}, \chi, \omega)$-module homomorphism

$$
\mathrm{d}_{\Pi_{\phi+(N+2) \chi / 2}^{\mathrm{R}}}^{\mathrm{R}}: \mathcal{V}\left(\Pi_{\chi}, R\left(\pi_{N}\right)\right) \rightarrow \mathcal{V}\left(\Pi, R\left(\pi_{N}\right)\right)
$$

Theorem 8.1. One has:

$$
\operatorname{sing} \mathrm{F}^{1} \mathcal{V}\left(\Pi, R\left(\pi_{n}\right)\right)=\mathrm{F}^{0} \mathcal{V}\left(\Pi, R\left(\pi_{n}\right)\right)+\mathrm{d}_{\Pi_{\phi+n \chi / 2}}^{n} \mathrm{~F}^{0} \mathcal{V}\left(\Pi_{\chi / 2}, R\left(\pi_{n-1}\right)\right)
$$




$$
+\mathrm{d}_{\Pi_{\phi+(2 N-n) \chi / 2}}^{2 N-n} \mathrm{~F}^{0} \mathcal{V}\left(\Pi_{\chi / 2}, R\left(\pi_{n+1}\right)\right), \quad 1 \leq n<N
$$

and

$$
\operatorname{sing} \mathrm{F}^{1} \mathcal{V}\left(\Pi, R\left(\pi_{N}\right)\right)=\mathrm{F}^{0} \mathcal{V}\left(\Pi, R\left(\pi_{N}\right)\right)+\mathrm{d}_{\Pi_{\phi+N \chi / 2}}^{N} \mathrm{~F}^{0} \mathcal{V}\left(\Pi_{\chi / 2}, R\left(\pi_{N-1}\right)\right)
$$

Proof. By the proof of Proposition 8.4, the $\mathfrak{d} \oplus \mathfrak{s p} \mathfrak{d}$-submodule $\mathrm{F}^{1} \mathcal{V}\left(\Pi, R\left(\pi_{n}\right)\right)$ is isomorphic to the direct sum

$$
\mathrm{F}^{0} \mathcal{V}\left(\Pi, R\left(\pi_{n}\right)\right) \oplus\left(\Pi_{\chi / 2} \otimes R\left(\pi_{n-1}\right)\right) \oplus\left(\Pi_{\chi / 2} \otimes R\left(\pi_{n+1}\right)\right) \oplus\left(\Pi_{\chi / 2} \otimes R\left(\pi_{n}+\pi_{1}\right)\right)
$$

where non-zero vectors in the last three summands have degree 1.

The $\mathfrak{s p} \mathfrak{d}$-module $R\left(\pi_{n}+\pi_{1}\right)$ satisfies the irreducibility criterion stated in Theorem 7.1, and its dimension is larger than $\operatorname{dim} R\left(\pi_{n}\right)$. As in [2, Lemma 7.8], if $\mathcal{V}\left(\Pi, R\left(\pi_{n}\right)\right.$ ) contained any singular vector of degree 1 lying in this summand, the $H$-submodule (freely) generated by this summand would be $H(\mathfrak{d}, \chi, \omega)$-stable and have a larger rank than $\mathcal{V}\left(\Pi, R\left(\pi_{n}\right)\right)$ itself, thus yielding a contradiction. We may thus conclude that no singular vectors of degree 1 have a non-zero projection to this summand.

However, by exactness of the conformally symplectic pseudo de Rham complex, the differentials $\mathrm{d}_{\Pi_{\phi+n \chi / 2}}^{n}$ with $n>1$ map $\mathrm{F}^{0} \mathcal{V}\left(\Pi_{\chi / 2}, R\left(\pi_{n-1}\right)\right.$ ) to non-zero (otherwise $\mathrm{d}_{\Pi_{\phi+n \chi / 2}}^{n}$ would vanish) non-constant (otherwise $\mathrm{d}_{\Pi_{\phi+n \chi / 2}}^{n}$ would be surjective) irreducible $\mathfrak{d} \oplus \mathfrak{s p} \mathfrak{d}$ summands of singular vectors, isomorphic to $\Pi_{\chi / 2} \otimes R\left(\pi_{n-1}\right)$. A similar argument applies to $\mathrm{d}_{\Pi_{\phi+(2 N-n) \chi / 2}}^{2 N-n}$ as well. These summands must lie in $\mathrm{F}^{1} \mathcal{V}\left(\Pi, R\left(\pi_{n}\right)\right)$, due to Remark 6.8. Now Proposition 8.4 shows that those are the only singular vectors of degree 1 , up to constant elements.

\subsubsection{Singular vectors of degree one, non-graded case}

Our setting is now the following: we have a tensor $H(\mathfrak{d}, \chi, \omega)$-module $\mathcal{V}\left(\Pi^{\prime}, R\left(\pi_{n}\right)\right)$ with $1 \leq n \leq N$, on which $c \in \mathfrak{d}^{\prime}$ acts non-trivially; here $n$ is fixed once and for all. Consequently, by Lemma 3.8, $\chi=0, \omega=\mathrm{d} \zeta$ for some $\zeta \in \mathfrak{d}^{*}$, and $\mathfrak{d}^{\prime}$ is trivial as a central extension of $\mathfrak{d}$. Explicitly, $\mathfrak{d}^{\prime}=\mathfrak{d}^{\zeta} \oplus \mathbf{k} c$, where $\mathfrak{d}^{\zeta}$ is spanned by elements $\partial_{\zeta}:=\partial+\zeta(\partial)$ for $\partial \in \mathfrak{d}$. Then $\Pi^{\prime}$ is isomorphic to $\Pi \otimes \mathbf{k}_{\lambda}$, where $\Pi$ is the restriction of $\Pi^{\prime}$ to $\mathfrak{d}^{\zeta}$, and $\mathbf{k}_{\lambda}$ is the 1-dimensional representation of the abelian Lie algebra $\mathbf{k} c$ on which $c$ acts via multiplication by $\lambda$. We may rewrite (6.5) as follows:

$$
\begin{aligned}
e *(1 \otimes u) & =\sum_{i, j=1}^{2 N}\left(\partial_{i} \partial_{j} \otimes 1\right) \otimes_{H}\left(1 \otimes \rho_{R}\left(f^{i j}\right) u\right) \\
& -\sum_{k=1}^{2 N}\left(\partial_{k} \otimes 1\right) \otimes_{H}\left(1 \otimes \rho_{R}\left(\partial_{\zeta}^{k}+\operatorname{ad}^{\mathfrak{s p}} \partial^{k}\right) u-\partial^{k} \otimes u\right) \\
& +((\ell+1) \otimes 1) \otimes_{H}(1 \otimes \lambda u), \quad u \in R,
\end{aligned}
$$

where $\ell:=\sum_{k} \zeta\left(\partial^{k}\right) \partial_{k}$. 
We now identify all $\Pi \otimes \mathbf{k}_{\lambda}$ with different $\lambda \in \mathbf{k}$ as vector spaces, and consequently all $\mathcal{V}\left(\Pi \otimes \mathbf{k}_{\lambda}, R\left(\pi_{n}\right)\right)$ with each other. Notice that $\mathcal{V}\left(\Pi \otimes \mathbf{k}_{0}, R\left(\pi_{n}\right)\right)$ is nothing but $\mathcal{V}\left(\Pi, R\left(\pi_{n}\right)\right):=\mathcal{V}\left(\pi^{*} \Pi, R\left(\pi_{n}\right)\right)$, and the first two terms in the right-hand side of (8.4) describe $e *(1 \otimes u)$ when $\lambda=0$.

Proposition 8.5. Let $V=\mathcal{V}\left(\Pi \otimes \mathbf{k}_{\lambda}, R\left(\pi_{n}\right)\right)$. Then the intersection $\operatorname{sing} V \cap \mathrm{F}^{1} V$ is independent of $\lambda$.

Proof. We show that each singular vector of degree 1 in $\mathcal{V}\left(\Pi \otimes \mathbf{k}_{0}, R\left(\pi_{n}\right)\right)$ is also a singular vector in $\mathcal{V}\left(\Pi \otimes \mathbf{k}_{\lambda}, R\left(\pi_{n}\right)\right)$, as the converse is completely analogous. Assume that $v$ is a singular vector of degree 1 , as in (8.3), for $\mathcal{V}\left(\Pi \otimes \mathbf{k}_{0}, R\left(\pi_{n}\right)\right)$. We need to compute $e * v$ in $\mathcal{V}\left(\Pi \otimes \mathbf{k}_{\lambda}, R\left(\pi_{n}\right)\right)$ and check that it lies in $\left(\mathrm{F}^{2} H \otimes \mathbf{k}\right) \otimes_{H} V$. However, the contribution in $e * v$ from the first two summands in the right-hand side of (8.4) belongs to $\left(\mathrm{F}^{2} H \otimes \mathbf{k}\right) \otimes_{H} V$ as $v$ is a singular vector for the $\lambda=0$ case; furthermore, the third summand left-straightens to $\left(\mathrm{F}^{2} H \otimes \mathbf{k}\right) \otimes_{H} V$ because $v$ is of degree 1 .

\subsection{Singular vectors of degree two in $\mathcal{V}\left(\Pi^{\prime}, R\left(\pi_{n}\right)\right), 1 \leq n \leq N$}

Throughout this section, we will focus on a tensor $H(\mathfrak{d}, \chi, \omega)$-module of the form $\mathcal{V}(R)=\mathcal{V}\left(\Pi^{\prime}, R\left(\pi_{n}\right)\right)$ for $n=1, \ldots, N$, and assume it contains a singular vector

$$
v=\sum_{i, j=1}^{2 N} \partial_{i} \partial_{j} \otimes v^{i j}+\sum_{k=1}^{2 N} \partial_{k} \otimes v^{k}+1 \otimes v^{0}, \quad v^{i j}, v^{k} \in R
$$

of degree 2. Recall the definition of $\psi(u)$ given in (7.1), and the representation $\rho_{\text {sing }}$ of $\mathfrak{d}^{\prime} \oplus \mathfrak{s p} \mathfrak{d}$ on the space of singular vectors (see Definition 6.1).

Lemma 8.3. We have

$$
\rho_{\text {sing }}\left(f^{\alpha \beta}\right) v=\psi\left(v^{\alpha \beta}\right) \bmod \mathrm{F}^{1} \mathcal{V}(R)
$$

In particular, $\mathcal{V}(R)$ contains a singular vector of degree two coinciding with $\psi(u)$ $\bmod \mathrm{F}^{1} \mathcal{V}(R)$ for some $u \in R$.

Proof. We can compute $e * v$ using $H$-linearity, (8.5) and (7.2). Comparing the result with (7.3), we obtain equation (8.6).

Now, as the vector $v$ is of degree 2, some $v^{i j}$ must be non-zero. Then $\psi\left(v^{i j}\right)$ is also nonzero, as the action of $\mathfrak{s p} \mathfrak{d}$ on $R$ is non-trivial, hence $\rho_{\text {sing }}\left(f^{i j}\right) v=\psi\left(v^{i j}\right) \bmod \mathrm{F}^{1} \mathcal{V}(R)$ is non-zero. This shows, in one shot, that the action of $\mathfrak{s p} \mathfrak{d}$ on each singular vector $v$ of degree 2 is non-trivial, and that for some $0 \neq u \in R$ there exists a singular vector coinciding with $\psi(u)$ modulo $\mathrm{F}^{1} \mathcal{V}(R)$, as the $\mathfrak{s p} \mathfrak{d}$-action preserves singular vectors, hence $\rho_{\text {sing }}\left(f^{i j}\right) v$ is singular too. 
Lemma 8.4. For $u \in R$, let $\phi(u)$ denote a singular vector, if there exists one, coinciding with $\psi(u) \bmod \mathrm{F}^{1} \mathcal{V}(R)$. Then $\rho_{\operatorname{sing}}\left(f^{\alpha \beta}\right) \phi(u)$ and $\rho_{\operatorname{sing}}(\widehat{\partial}) \phi(u)$ are also singular vectors, which coincide respectively with $\psi\left(f^{\alpha \beta}(u)\right)$ and $\psi(\widehat{\partial} \cdot u+\chi(\partial) u)$ modulo $\mathrm{F}^{1} \mathcal{V}(R)$.

Proof. The claims follow from Lemma 8.3 and Lemma 6.7, along with the definition of $\psi(u)$.

Lemma 8.5. For every $u \in R$, there exists some singular vector $\phi(u)$ coinciding with $\psi(u)$ modulo $\mathrm{F}^{1} \mathcal{V}(R)$. The corresponding map $\phi: R \rightarrow \operatorname{gr}^{2} \mathcal{V}(R)$ is a linear homomorphism and satisfies:

$$
\begin{aligned}
\rho_{\text {sing }}\left(f^{\alpha \beta}\right) \phi(u) & =\phi\left(f^{\alpha \beta} \cdot u\right), \\
\rho_{\text {sing }}\left(f^{\alpha \beta}\right) v & =\phi\left(v^{\alpha \beta}\right), \\
\rho_{\text {sing }}(\widehat{\partial}) \phi(u) & =\phi(\widehat{\partial} \cdot u+\chi(\partial) u), \\
\rho_{\text {sing }}(c) \phi(u) & =\phi(c \cdot u),
\end{aligned}
$$

for all $u \in R$ and $v \in \operatorname{sing} \mathcal{V}(R)$ is as in (8.5). Thus, $\phi$ is a well-defined injective homomorphism that commutes with the $\mathfrak{s p} \mathfrak{d}$-action.

Proof. The space of elements $u \in R$ for which we can locate a singular vector equal to $\psi(u)$ modulo $\mathrm{F}^{1} \mathcal{V}(R)$ is non-zero by Lemma 8.3, and is $\mathfrak{d}^{\prime} \oplus \mathfrak{s p} \mathfrak{d}$-stable. By irreducibility of $R$, this space must be all of $R$. Injectivity follows from (8.8), whereas the other properties have already been proved.

Corollary 8.1. The space of singular vectors of degree 2 does not contain trivial $\mathfrak{s p d}$ summands.

Proof. If $v$ is a homogeneous singular vector of degree 2 lying in a trivial summand, then $0=\rho_{\text {sing }}\left(f^{\alpha \beta}\right) v=\phi\left(v^{\alpha \beta}\right)$ for all $\alpha, \beta$. But $\phi$ is injective, hence $v^{\alpha \beta}=0$, a contradiction with $v$ being of degree 2 , hence $v=0$.

We are now ready to classify singular vectors of degree two. As before, we will first proceed with tensor modules on which the action of $c \in \mathfrak{d}^{\prime}$ is trivial, and then extend our results to the general case.

\subsubsection{Singular vectors of degree two, graded case}

Our setting is now as follows: $\Pi$ is a $\mathfrak{d}$-module, and $\mathcal{V}\left(\Pi, R\left(\pi_{n}\right)\right):=\mathcal{V}\left(\pi^{*} \Pi, R\left(\pi_{n}\right)\right)=$ $\mathcal{V}\left(\Pi^{\prime}, R\left(\pi_{n}\right)\right)$ denotes the tensor $H(\mathfrak{d}, \chi, \omega)$-module as in Definition 6.2(iii).

Proposition 8.6. Non-zero singular vectors of degree 2 in $\mathcal{V}\left(\Pi, R\left(\pi_{n}\right)\right)$ project to a unique $\mathfrak{d} \oplus \mathfrak{s p} \mathfrak{d}$-irreducible summand in $\operatorname{gr}^{2} \mathcal{V}\left(\Pi, R\left(\pi_{n}\right)\right)$, which is isomorphic to $\Pi_{\chi} \otimes R\left(\pi_{n}\right)$. Moreover, the homomorphism $\mathrm{d}_{\Pi_{\phi+n \chi / 2}}^{n} \mathrm{~d}_{\Pi_{\phi+(2 N-n+2) \chi / 2}}^{2 N-n+1}$ maps injectively $\mathrm{F}^{0} \mathcal{V}\left(\Pi_{\chi}, R\left(\pi_{n}\right)\right)$ 
to an irreducible summand of singular vectors of degree 2 in $\mathcal{V}\left(\Pi, R\left(\pi_{n}\right)\right)$. In particular, the space of non-zero homogeneous singular vectors of degree 2 in $\mathcal{V}\left(\Pi, R\left(\pi_{n}\right)\right)$ coincides with $\mathrm{d}_{\Pi_{\phi+n \chi / 2}}^{n} \mathrm{~d}_{\Pi_{\phi+(2 N-n+2) \chi / 2}}^{2 N-n+1} \mathrm{~F}^{0} \mathcal{V}\left(\Pi_{\chi}, R\left(\pi_{n}\right)\right)$.

Proof. The first claim is clear. As for the second claim, an $H(\mathfrak{d}, \chi, \omega)$-module homomorphism maps singular vectors to singular vectors, and its restriction to an irreducible $\mathfrak{d} \oplus$ $\mathfrak{s p} \mathfrak{d}$-summand is either 0 or an isomorphism. Recall that, by exactness of the conformally symplectic pseudo de Rham complex, $\mathrm{d}_{\Pi_{\phi+n \chi / 2}}^{n} \mathrm{~d}_{\Pi_{\phi+n \chi / 2}}^{n-1}$ equals 0 , and $\operatorname{ker} \mathrm{d}_{\Pi_{\phi+n \chi / 2}}^{n}=$ Im $\mathrm{d}_{\Pi_{\phi+n \chi / 2}}^{n-1}$. Now, $\mathrm{d}_{\Pi_{\phi+n \chi / 2}}^{n-1}$ must be injective on $\mathrm{d}_{\Pi_{\phi+(2 N-n+2) \chi / 2}}^{2 N-n+1} \mathrm{~F}^{0} \mathcal{V}\left(\Pi_{\chi}, R\left(\pi_{n}\right)\right)$. If it were not, this homogeneous summand of degree 1 singular vectors would have to lie in the image of $\mathrm{d}_{\Pi_{\phi+n \chi / 2}}^{n-1}$, hence it would be obtained by applying $\mathrm{d}_{\Pi_{\phi+n \chi / 2}}^{n-1}$ to constant singular vectors. However, $\mathrm{d}_{\Pi_{\phi+n \chi / 2}}^{n-1} \mathrm{~F}^{0} \mathcal{V}\left(\Pi_{\chi}, R\left(\pi_{n-2}\right)\right) \simeq \Pi_{\chi} \otimes R\left(\pi_{n-2}\right)$ as $\mathfrak{d} \oplus \mathfrak{s p} \mathfrak{d}$-modules, yielding a contradiction. We conclude that $\mathrm{d}_{\Pi_{\phi+n \chi / 2}}^{n} \mathrm{~d}_{\Pi_{\phi+(2 N-n+2) \chi / 2}}^{2 N-n+1} \mathrm{~F}^{0} \mathcal{V}\left(\Pi_{\chi}, R\left(\pi_{n}\right)\right)$ is a homogeneous summand of degree 2 singular vectors, which is $\mathfrak{d} \oplus \mathfrak{s p} \mathfrak{d}$-isomorphic to $\Pi_{\chi} \otimes R\left(\pi_{n}\right)$.

Remark 8.2. Note that, if $\chi=0$, then a proper submodule $M$ of a tensor module $\mathcal{V}(R)$ can in principle contain non-homogeneous singular vectors of degree 2 . Indeed, homogeneous singular vectors of degree 0 and 2 span in such a case isomorphic $\mathfrak{d} \oplus \mathfrak{s p} \mathfrak{d}$-submodules of $\mathcal{V}(R)$, so that suitable linear combinations will yield possibly non-homogeneous singular vectors. We will see below that singular vectors of degree 2 contained in a proper submodule are always homogenous. We will show this by constructing, when $\chi=0$, non-homogeneous automorphisms of reducible tensor modules.

\subsubsection{Singular vectors of degree two, non-graded case}

Our setting is as above in Section 8.4.2, and again we identify all $\mathcal{V}\left(\Pi \otimes \mathbf{k}_{\lambda}, R\left(\pi_{n}\right)\right)$ with each other for $\lambda \in \mathbf{k}$.

Proposition 8.7. Let $v$ be as in (8.5) with $v^{i j}=\rho_{R}\left(f^{i j}\right) u$ for some $u \in R$. Then $v$ is singular in $\mathcal{V}\left(\Pi \otimes \mathbf{k}_{0}, R\left(\pi_{n}\right)\right)$ if and only if $v_{\lambda}:=v+\lambda \ell \otimes u$ is singular in $V=$ $\mathcal{V}\left(\Pi \otimes \mathbf{k}_{\lambda}, R\left(\pi_{n}\right)\right), \lambda \in \mathbf{k}$, where $\ell=\sum_{k} \zeta\left(\partial^{k}\right) \partial_{k}$.

Proof. Proceed as in the proof of Proposition 8.5. The only two terms that do not obviously lie in $\left(\mathrm{F}^{2} H \otimes \mathbf{k}\right) \otimes_{H} V$ cancel with each other modulo $\left(\mathrm{F}^{2} H \otimes \mathbf{k}\right) \otimes_{H} V$.

If $W \subset \mathcal{V}(R)$ is a subset of singular vectors of degree 2, we will denote by $W_{\lambda}$ the set of all $v_{\lambda}$ defined as in Proposition 8.7 above, where $v \in W$.

\subsection{Classification of singular vectors}

At last, we show that there are no further singular vectors to compute. 
Proposition 8.8. Let $R=\Pi^{\prime} \otimes U$ be a finite-dimensional irreducible representation of $\mathfrak{d}^{\prime} \oplus \mathfrak{s p \mathfrak { d }}$ such that $\mathcal{V}(R)$ admits non-constant singular vectors. Then $U \simeq R\left(\pi_{i}\right)$ for some $0 \leq i \leq N$.

Proof. We already know from Proposition 8.3 that the degree of non-constant singular vectors in $\mathcal{V}(R)$ is either 1 or 2 . Let us treat the case of degree 2 first.

If $\mathcal{V}(R)$ contains singular vectors of degree 2, we know by Lemma 8.3 that it must then contain at least one singular vector $\phi(u) \in \mathcal{V}(R)$ of degree 2 satisfying $\phi(u) \equiv$ $\psi(u) \bmod \mathrm{F}^{1} \mathcal{V}(R)$ for some $0 \neq u \in R$, hence for all $u \in R$ by Lemma 8.5 and $\mathfrak{d}^{\prime} \oplus \mathfrak{s p} \mathfrak{d}$ irreducibility of $R$. Now, $e * \phi(u) \in\left(\mathrm{F}^{2} H \otimes \mathbf{k}\right) \otimes_{H} \mathcal{V}(R)$ by singularity of $\phi(u)$ and

$$
\begin{aligned}
e * \mathrm{~F}^{1} \mathcal{V}(R) & \subset\left(\mathrm{F}^{2} H \otimes \mathrm{F}^{1} H\right) \otimes_{H} \mathcal{V}(R) \\
& \subset \mathrm{F}^{3}(H \otimes H) \otimes_{H} \mathcal{V}(R)=\left(\mathrm{F}^{3} H \otimes \mathbf{k}\right) \otimes_{H} \mathcal{V}(R),
\end{aligned}
$$

by $H$-bilinearity. Adding up, we obtain $e * \psi(u) \in\left(\mathrm{F}^{3} H \otimes \mathbf{k}\right) \otimes_{H} \mathcal{V}(R)$ and we may use Lemma 7.3 to conclude.

Let us proceed now with the degree 1 case. If $v$ is a non-zero singular vector of degree 1 as in (8.3), we may assume without loss of generality that $v$ lies in a $\mathfrak{d}^{\prime} \oplus \mathfrak{s p} \mathfrak{d}$-irreducible component $W$ of $\operatorname{sing} \mathcal{V}(R)$ projecting injectively to $\operatorname{gr}^{1} \mathcal{V}(R)$, which is isomorphic to $\mathfrak{d} \otimes R$ as an $\mathfrak{s p} \mathfrak{d}$-module. The coefficient multiplying $1 \otimes \partial_{i}$ in the right-straightened expression for $e * v$ equals $\psi\left(v^{i}\right) \bmod \mathrm{F}^{1} \mathcal{V}(R)$, by Lemma 7.1. On the other hand, equation (7.3) shows that it lies in $\mathrm{F}^{1} H \cdot \operatorname{sing} \mathcal{V}(R)$. However, $\mathrm{F}^{1} \mathcal{V}(R) \subset \mathrm{F}^{1} H \cdot \operatorname{sing} \mathcal{V}(R)$ and we conclude that

$$
\begin{aligned}
e * \psi\left(s^{i}\right) & \in\left(\mathbf{k} \otimes \mathrm{F}^{1} H\right) e * \operatorname{sing} \mathcal{V}(R) \\
& \subset\left(\mathrm{F}^{2} H \otimes \mathrm{F}^{1} H\right) \otimes_{H} \mathcal{V}(R)=\left(\mathrm{F}^{3} H \otimes \mathbf{k}\right) \otimes_{H} \mathcal{V}(R) .
\end{aligned}
$$

Observe now that $\psi$ is $\mathbf{k}$-linear and that the subspace of $R$ which is $\mathbf{k}$-linearly spanned by all $v^{i}$ thus obtained coincides with the (non-zero!) image of the restriction to $\mathfrak{d}^{*} \otimes W$ of the $\mathfrak{s p} \mathfrak{d}$-homomorphism

$$
\mathfrak{d}^{*} \otimes \operatorname{gr}^{1} \operatorname{sing} \mathcal{V}(R) \simeq \mathfrak{d}^{*} \otimes(\mathfrak{d} \otimes R) \rightarrow R
$$

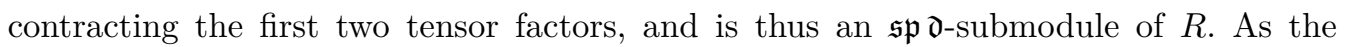
$\mathfrak{s p} \mathfrak{d}$-module $R$ is $U$-isotypical, we may again use Lemma 7.3 and conclude.

As a result of the above discussion, we obtain the following classification of singular vectors.

Theorem 8.2. Let $V=\mathcal{V}\left(\Pi^{\prime}, U\right)$ be a tensor module over $H(\mathfrak{d}, \chi, \omega)$, where $\Pi^{\prime} \otimes U$ is an irreducible $\mathfrak{d}^{\prime} \oplus \mathfrak{s p} \mathfrak{d}$-module in which $c \in \mathfrak{d}^{\prime}$ acts via multiplication by $\lambda \in \mathbf{k}$. When $\lambda \neq 0$, denote by $\Pi$ the unique $\mathfrak{d}$-module such that $\Pi^{\prime} \simeq \Pi \nabla \mathbf{k}_{\lambda}$ as modules over $\mathfrak{d}^{\prime}=\mathfrak{d}^{\zeta} \oplus \mathbf{k} c$; when $\lambda=0$, choose $\Pi$ so as to satisfy $\Pi^{\prime}=\pi^{*} \Pi$. 
- If $U \simeq R\left(\pi_{0}\right)=\mathbf{k}$, then

$$
\operatorname{sing} V=\mathrm{F}^{1} V=\mathrm{F}^{0} V \oplus \mathrm{d}_{\Pi_{\phi+N \chi}}^{2 N} \mathrm{~F}^{0} \mathcal{V}\left(\Pi_{\chi / 2}, R\left(\pi_{1}\right)\right) \text {. }
$$

- If $U \simeq R\left(\pi_{n}\right), 1 \leq n<N$, then

$$
\begin{aligned}
\operatorname{sing} V=\mathrm{F}^{0} V & \oplus \mathrm{d}_{\Pi_{\phi+n \chi / 2}}^{n} \mathrm{~F}^{0} \mathcal{V}\left(\Pi_{\chi / 2}, R\left(\pi_{n-1}\right)\right) \\
& \oplus \mathrm{d}_{\Pi_{\phi+(2 N-n) \chi / 2}}^{2 N-n} \mathrm{~F}^{0} \mathcal{V}\left(\Pi_{\chi / 2}, R\left(\pi_{n+1}\right)\right) \\
& \oplus\left(\mathrm{d}_{\Pi_{\phi+n \chi / 2}}^{n} \mathrm{~d}_{\Pi_{\phi+(2 N-n+2) \chi / 2} N-n+1}^{2 N} \mathrm{~F}^{0} \mathcal{V}\left(\Pi_{\chi}, R\left(\pi_{n}\right)\right)\right)_{\lambda} .
\end{aligned}
$$

- If $U \simeq R\left(\pi_{N}\right)$, then

$$
\begin{aligned}
\operatorname{sing} V=\mathrm{F}^{0} V & \oplus \mathrm{d}_{\Pi_{\phi+N \chi / 2}}^{N} \mathrm{~F}^{0} \mathcal{V}\left(\Pi_{\chi / 2}, R\left(\pi_{N-1}\right)\right) \\
& \oplus\left(\mathrm{d}_{\Pi_{\phi+N \chi / 2}}^{N} \mathrm{~d}_{\Pi_{\phi+(N+2) \chi / 2}^{N+1}}^{N+1} \mathrm{~F}^{0} \mathcal{V}\left(\Pi_{\chi}, R\left(\pi_{N}\right)\right)\right)_{\lambda} .
\end{aligned}
$$

- $\operatorname{sing} V=\mathrm{F}^{0} V$ in all other cases.

Proof. We know from Propositions 8.3 and 8.8 that singular vectors in $\mathcal{V}\left(\Pi^{\prime}, U\right)$ are of degree at most 2 and only occur when $U \simeq R\left(\pi_{n}\right), 0 \leq n \leq N$. Furthermore, Lemma 6.4 shows that all vectors of degree 0 are singular. Non-constant singular vectors of $\mathcal{V}\left(\Pi^{\prime}, \mathbf{k}\right)$ are computed in Section 8.1, and all other non-constant singular vectors are classified in Sections 8.4 and 8.5.

Remark 8.3. It is not difficult to show that singular vectors of degree two may also be obtained as $\mathrm{d}_{\Pi_{\phi+(2 N-n) \chi / 2}}^{2 N-n} \mathrm{~d}_{\Pi_{\phi+(n+2) \chi / 2}}^{n+1} \mathrm{~F}^{0} \mathcal{V}\left(\Pi_{\chi}, R\left(\pi_{n}\right)\right)$ in the tensor module $\mathcal{V}\left(\Pi, R\left(\pi_{n}\right)\right), 1 \leq n<N$, and as $\mathrm{d}_{\Pi_{\phi+(N+2) \chi / 2}^{\mathrm{R}}} \mathrm{F}^{0} \mathcal{V}\left(\Pi_{\chi}, R\left(\pi_{N}\right)\right)$ in $\mathcal{V}\left(\Pi_{\chi}, R\left(\pi_{N}\right)\right)$. This shows that $\mathrm{d}_{\Pi_{\phi+n \chi / 2}}^{n} \mathrm{~d}_{\Pi_{\phi+(2 N-n+2) \chi / 2}^{2 N-n+1}}^{2 N}$ and $\mathrm{d}_{\Pi_{\phi+(2 N-n) \chi / 2}^{2 N-n}}^{2 N} \mathrm{~d}_{\Pi_{\phi+(n+2) \chi / 2}}^{n+1}$ (respectively, $\mathrm{d}_{\Pi_{\phi+N \chi / 2}}^{N} \mathrm{~d}_{\Pi_{\phi+(N+2) \chi / 2}+1}^{N+1}$ and $\left.\mathrm{d}_{\Pi_{\phi+(N+2) \chi / 2}}^{\mathrm{R}}\right)$ coincide up to multiplication by a non-zero scalar.

\section{Submodules of tensor modules, graded case}

In this section we will show that the only non-zero proper submodules of tensor modules appearing in a twisted conformally symplectic pseudo de Rham complex are either the image of a differential or the sum or intersection of two such images. Notice that the action of $\mathcal{P}$ on all such modules factors through $\mathcal{H}$, so that it makes sense to talk of homogeneous singular vectors. Throughout this section, $\Pi$ is an irreducible finitedimensional $\mathfrak{d}$-module and $\mathcal{V}(\Pi, U)$ denotes the tensor $H(\mathfrak{d}, \chi, \omega)$-module $\mathcal{V}\left(\pi^{*} \Pi, U\right)$. 


\subsection{Automorphisms of tensor modules}

Let $f: V \rightarrow V$ be a homomorphism of tensor modules. Then $f$ maps constant vectors in $V$, which are singular, to $\operatorname{sing} V$. If $f$ is non-zero, then $\left.f\right|_{\mathrm{F}^{0} V}$ must be an isomorphism. This is typically possible only when $f$ is a multiple of the identity. However, the case of reducible tensor modules over $H(\mathfrak{d}, \chi, \omega)$ is a remarkable exception, when $\chi=0$.

Theorem 9.1. Let $V=\mathcal{V}\left(\Pi, R\left(\pi_{n}\right)\right)$ be a tensor $H(\mathfrak{d}, 0, \omega)$-module for $1 \leq n \leq N$. Then each $H(\mathfrak{d}, 0, \omega)$-endomorphism of $V$ is of the form

$$
f_{\alpha \beta}=\alpha \mathrm{id}_{V}+\beta \mathrm{d}_{\Pi_{\phi}}^{n} \mathrm{~d}_{\Pi_{\phi}}^{2 N-n+1}
$$

for some $\alpha, \beta \in \mathbf{k}$. The endomorphism $f_{\alpha \beta}$ is invertible if and only if $\alpha \neq 0$.

Proof. The maps $\mathrm{id}_{V}$ and $\mathrm{d}_{\Pi_{\phi}}^{n} \mathrm{~d}_{\Pi_{\phi}}^{2 N-n+1}$ are commuting $H(\mathfrak{d}, 0, \omega)$-endomorphisms, and so are their linear combinations. As $\mathrm{id}_{V}$ is invertible and $\mathrm{d}_{\Pi_{\phi}}^{n} \mathrm{~d}_{\Pi_{\phi}}^{2 N-n+1}$ is nilpotent, the invertibility claim follows.

As for showing that any $H(\mathfrak{d}, 0, \omega)$-homomorphism $f: V \rightarrow V$ is necessarily of this form, notice that if $f$ is non-zero, then it must map $\mathrm{F}^{0} V \simeq \Pi \otimes R\left(\pi_{n}\right)$ to an irreducible $\mathfrak{d} \oplus \mathfrak{s p} \mathfrak{d}$-component of $\operatorname{sing} V$. However, since $\chi=0$, the constant and the degree 2 summands are isomorphic.

Composing $f$ with projections to components of degree 0 and 2 yields $\mathfrak{d} \oplus \mathfrak{s p} \mathfrak{d}$ homomorphisms which, by irreducibility of $\mathrm{F}^{0} V$ and Schur's Lemma, must be scalar multiples of the restrictions of $\operatorname{id}_{V}$ and $\mathrm{d}_{\Pi_{\phi}}^{n} \mathrm{~d}_{\Pi_{\phi}}^{2 N-n+1}$ to $\mathrm{F}^{0} V$.

When $\chi \neq 0$, the irreducible $\mathfrak{d} \oplus \mathfrak{s p} \mathfrak{d}$-summands of sing $V$ are pairwise non-isomorphic, hence homogeneous. When $\chi=0$, however, non-homogeneous singular vectors only occur in some $f_{\alpha \beta} \mathrm{F}^{0} V$, where $\alpha$ and $\beta$ are both non-zero. In this case, as $V$ is generated over $H$ by its constant singular vectors, $V=f_{\alpha \beta}(V)$ is also $H$-generated by $f_{\alpha \beta} \mathrm{F}^{0} V$. Hence, if $M \subset V$ is an $H(\mathfrak{d}, 0, \omega)$-submodule containing $f_{\alpha \beta} \mathrm{F}^{0} V$ with $\alpha \neq 0$, then $M=V$. This proves the following claim:

Proposition 9.1. Every proper submodule of a tensor module $\mathcal{V}\left(\Pi, R\left(\pi_{n}\right)\right), 1 \leq n \leq N$, contains the homogenous components of all of its singular vectors.

\subsection{Classification of submodules of tensor modules}

Throughout this section, we will make repeated use of the standard homomorphism theorem in the following form: if $f: M \rightarrow N$ is a morphism of modules and $M_{0} \subset M$ is a submodule, then

$$
f\left(M_{0}\right) \simeq\left(M_{0}+\operatorname{ker} f\right) / \operatorname{ker} f \simeq M_{0} /\left(M_{0} \cap \operatorname{ker} f\right)
$$


Our goal is to provide an explicit description of submodules of $\mathcal{V}\left(\Pi, R\left(\pi_{n}\right)\right)$ in terms of the differentials in the conformally symplectic pseudo de Rham complex (5.37). Recall that $\mathcal{T}(\Pi, U)=\mathcal{V}\left(\Pi_{-\phi}, U\right)$, by Proposition 6.4, so the relevant maps are:

$$
\begin{array}{rlrl}
\mathrm{d}_{\Pi_{\phi+n \chi / 2}}^{n}: \mathcal{V}\left(\Pi_{\chi / 2}, R\left(\pi_{n-1}\right)\right) & \rightarrow \mathcal{V}\left(\Pi, R\left(\pi_{n}\right)\right), & & 1 \leq n \leq N, \\
\mathrm{~d}_{\Pi_{\phi+(2 N-n) \chi / 2}}^{2 N-n}: \mathcal{V}\left(\Pi_{\chi / 2}, R\left(\pi_{n+1}\right)\right) \rightarrow \mathcal{V}\left(\Pi, R\left(\pi_{n}\right)\right), & & 0 \leq n<N, \\
\mathrm{~d}_{\Pi_{\phi+(N+2) \chi / 2}^{\mathrm{R}}}^{\mathrm{R}}: \mathcal{V}\left(\Pi_{\chi}, R\left(\pi_{N}\right)\right) \rightarrow \mathcal{V}\left(\Pi, R\left(\pi_{N}\right)\right), & \\
\mathrm{d}_{\Pi_{\phi+n \chi / 2}}^{n} \mathrm{~d}_{\Pi_{\phi+(2 N-n+2) \chi / 2}}^{2 N-n+1}: \mathcal{V}\left(\Pi_{\chi}, R\left(\pi_{n}\right)\right) \rightarrow \mathcal{V}\left(\Pi, R\left(\pi_{n}\right)\right), & & 1 \leq n \leq N .
\end{array}
$$

Observe that (9.1) and (9.2) map constant vectors to singular vectors of degree 1, thus increasing the degree by 1 , whereas (9.3) and (9.4) are nonzero and homogeneous of degree 2. Moreover, Schur's Lemma forces the map $\mathrm{d}_{\Pi_{\phi+(N+2) \chi / 2}}^{\mathrm{R}}$ to coincide with $\mathrm{d}_{\Pi_{\phi+N \chi / 2}}^{N} \mathrm{~d}_{\Pi_{\phi+(N+2) \chi / 2}+1}^{N+1}$ up to a nonzero multiplicative constant, as they both map constant vectors in $\mathcal{V}\left(\Pi_{\chi}, R\left(\pi_{N}\right)\right)$ to the same irreducible $\mathfrak{d} \oplus \mathfrak{s p} \mathfrak{d}$-summand of singular vectors of degree 2 .

Notice that (5.37) being a complex forces $\mathrm{d}_{\Pi}^{n+1} \mathrm{~d}_{\Pi}^{n}=0$ when $1 \leq n<2 N, n \neq N$, so that $\operatorname{Im} \mathrm{d}_{\Pi}^{n} \subset \operatorname{ker} \mathrm{d}_{\Pi}^{n+1}$. When furthermore $n \neq 2 N-1$, the inclusion becomes an equality due to exactness.

Lemma 9.1. If $1 \leq n \leq N$, then

$$
\begin{aligned}
& \operatorname{sing} \operatorname{Im} \mathrm{d}_{\Pi_{\phi+n \chi / 2}}^{n} \\
& \qquad=\mathrm{d}_{\Pi_{\phi+n \chi / 2}}^{n} \mathrm{~F}^{0} \mathcal{V}\left(\Pi_{\chi / 2}, R\left(\pi_{n-1}\right)\right) \oplus \mathrm{d}_{\Pi_{\phi+n \chi / 2}}^{n} \mathrm{~d}_{\Pi_{\phi+(2 N-n+2) \chi / 2}}^{2 N-n+1} \mathrm{~F}^{0} \mathcal{V}\left(\Pi_{\chi}, R\left(\pi_{n}\right)\right) .
\end{aligned}
$$

Proof. We know that

$$
\operatorname{sing} \mathcal{V}\left(\Pi_{\chi / 2}, R\left(\pi_{n-1}\right)\right) \supset \mathrm{F}^{0} \mathcal{V}\left(\Pi_{\chi / 2}, R\left(\pi_{n-1}\right)\right) \oplus \mathrm{d}_{\Pi_{\phi+(2 N-n+2) \chi / 2}}^{2 N-n+1} \mathrm{~F}^{0} \mathcal{V}\left(\Pi_{\chi}, R\left(\pi_{n}\right)\right)
$$

Applying $\mathrm{d}_{\Pi_{\phi+n \chi / 2}}^{n}$ to both sides and using Lemma 6.2, one obtains

$\operatorname{sing} \operatorname{Im} \mathrm{d}_{\Pi_{\phi+n \chi / 2}}^{n}$

$$
\supset \mathrm{d}_{\Pi_{\phi+n \chi / 2}}^{n} \mathrm{~F}^{0} \mathcal{V}\left(\Pi_{\chi / 2}, R\left(\pi_{n-1}\right)\right) \oplus \mathrm{d}_{\Pi_{\phi+n \chi / 2}}^{n} \mathrm{~d}_{\Pi_{\phi+(2 N-n+2) \chi / 2}}^{2 N-n+1} \mathrm{~F}^{0} \mathcal{V}\left(\Pi_{\chi}, R\left(\pi_{n}\right)\right)
$$

In order to show that the above inclusion is indeed an equality, recall Theorem 8.2 and observe that $\operatorname{Imd}_{\Pi_{\phi+n \chi / 2}}^{n}$ is a proper submodule of $\mathcal{V}\left(\Pi, R\left(\pi_{n}\right)\right)$, so it cannot contain constant singular vectors. Moreover, when $n<N$, the complex property shows that $\operatorname{Im} \mathrm{d}_{\Pi_{\phi+n \chi / 2}}^{n}$ cannot contain $\mathrm{d}_{\Pi_{\phi+(2 N-n) \chi / 2}}^{2 N-n} \mathrm{~F}^{0} \mathcal{V}\left(\Pi_{\chi / 2}, R\left(\pi_{n+1}\right)\right)$ as this is not killed by $\mathrm{d}_{\Pi_{\phi+n \chi / 2}^{n+1}}^{n+1}$. 
Lemma 9.2. For $1 \leq n \leq N$, we have

$\operatorname{sing} \operatorname{Im} \mathrm{d}_{\Pi_{\phi+(2 N-n) \chi / 2}}^{2 N-n}$

$$
=\mathrm{d}_{\Pi_{\phi+(2 N-n) \chi / 2}}^{2 N-n} \mathrm{~F}^{0} \mathcal{V}\left(\Pi_{\chi / 2}, R\left(\pi_{n+1}\right)\right) \oplus \mathrm{d}_{\Pi_{\phi+n \chi / 2}}^{n} \mathrm{~d}_{\Pi_{\phi+(2 N-n+2) \chi / 2}}^{2 N-n+1} \mathrm{~F}^{0} \mathcal{V}\left(\Pi_{\chi}, R\left(\pi_{n}\right)\right) .
$$

Proof. Similar to the proof of Lemma 9.1, using Remark 8.3.

Lemma 9.3. If $1 \leq n \leq N$, then

$$
\operatorname{sing} \operatorname{Im} \mathrm{d}_{\Pi_{\phi+n \chi / 2}}^{n} \mathrm{~d}_{\Pi_{\phi+(2 N-n+2) \chi / 2}}^{2 N-n+1}=\mathrm{d}_{\Pi_{\phi+n \chi / 2}}^{n} \mathrm{~d}_{\Pi_{\phi+(2 N-n+2) \chi / 2}}^{2 N-n+1} \mathrm{~F}^{0} \mathcal{V}\left(\Pi_{\chi}, R\left(\pi_{n}\right)\right)
$$

Proof. The homomorphism $\mathrm{d}_{\Pi_{\phi+n \chi / 2}}^{n} \mathrm{~d}_{\Pi_{\phi+(2 N-n+2) \chi / 2}}^{2 N-n+1}$ is homogeneous of degree 2, so the subspace sing $\operatorname{Im} \mathrm{d}_{\Pi_{\phi+n \chi / 2}}^{n} \mathrm{~d}_{\Pi_{\phi+(2 N-n+2) \chi / 2}}^{2 N-n+1}$ can only contain elements of degree $\geq 2$. As $\mathrm{d}_{\Pi_{\phi+n \chi / 2}}^{n} \mathrm{~d}_{\Pi_{\phi+(2 N-n+2) \chi / 2}}^{2 N-n+1}$ is non-zero, constant vectors are mapped non-trivially to singular vectors of degree two.

Proposition 9.2. The submodule $\operatorname{Im} \mathrm{d}_{\Pi_{\phi+n \chi / 2}}^{n} \mathrm{~d}_{\Pi_{\phi+(2 N-n+2) \chi / 2}}^{2 N-n+1}$ is irreducible for every $0<$ $n \leq N$.

Proof. As

$$
\operatorname{Im} \mathrm{d}_{\Pi_{\phi+n \chi / 2}}^{n} \mathrm{~d}_{\Pi_{\phi+(2 N-n+2) \chi / 2}}^{2 N-n+1}=\mathrm{d}_{\Pi_{\phi+n \chi / 2}}^{n} \mathrm{~d}_{\Pi_{\phi+(2 N-n+2) \chi / 2}}^{2 N-n+1} \mathcal{V}\left(\Pi_{\chi}, R\left(\pi_{n}\right)\right)
$$

it is generated as an $H$-module by $\mathrm{d}_{\Pi_{\phi+n \chi / 2}}^{n} \mathrm{~d}_{\Pi_{\phi+(2 N-n+2) \chi / 2}}^{2 N-n+1} \mathrm{~F}^{0} \mathcal{V}\left(\Pi_{\chi}, R\left(\pi_{n}\right)\right)$, which by Lemma 9.3 is an irreducible $\mathfrak{d} \oplus \mathfrak{s p} \mathfrak{d}$-summand containing all singular vectors. A non-zero submodule of $\operatorname{Im}_{\Pi_{\phi+n \chi / 2}}^{n} \mathrm{~d}_{\Pi_{\phi+(2 N-n+2) \chi / 2}}^{2 N-n+1}$ must then contain all of its singular vectors, which generate it over $H$.

Corollary 9.1. The submodule $\operatorname{Im} \mathrm{d}_{\Pi_{\phi+(N+2) \chi / 2}}^{\mathrm{R}}$ is irreducible.

Proof. As $\mathrm{d}_{\Pi_{\phi+(N+2) \chi / 2}}^{\mathrm{R}}$ coincides with $\mathrm{d}_{\Pi_{\phi+N \chi / 2}}^{N} \mathrm{~d}_{\Pi_{\phi+(N+2) \chi / 2}}^{N+1}$ up to a non-zero multiplicative constant, the claim follows from Proposition 9.2.

Proposition 9.3. If $1 \leq n<N$, then:

(1) $\operatorname{Im} \mathrm{d}_{\Pi_{\phi+n \chi / 2}}^{n} \cap \operatorname{Im}_{\Pi_{\phi+(2 N-n) \chi / 2}}^{2 N-n}$ is maximal in $\operatorname{Im} \mathrm{d}_{\Pi_{\phi+n \chi / 2}}^{n}$;

(2) $\operatorname{Im} \mathrm{d}_{\Pi_{\phi+n \chi / 2}}^{n} \cap \operatorname{Im~}_{\Pi_{\phi+(2 N-n) \chi / 2}}^{2 N-n}$ is maximal in $\operatorname{Im~}_{\Pi_{\phi+(2 N-n) \chi / 2}}^{2 N-n}$;

(3) $\operatorname{Im} \mathrm{d}_{\Pi_{\phi+n \chi / 2}}^{n}$ is maximal in $\operatorname{Im} \mathrm{d}_{\Pi_{\phi+n \chi / 2}}^{n}+\operatorname{Im~}_{\Pi_{\phi+(2 N-n) \chi / 2}}^{2 N-n}$;

(4) $\operatorname{Im~}_{\Pi_{\phi+(2 N-n) \chi / 2}}^{2 N-n}$ is maximal in $\operatorname{Im~}_{\Pi_{\phi+n \chi / 2}}^{n}+\operatorname{Im~}_{\Pi_{\phi+(2 N-n) \chi / 2}}^{2 N-n}$. 
Proof. One has

$$
\begin{aligned}
\operatorname{Im~}_{\Pi_{\phi+(2 N-n) \chi / 2}}^{2 N-n} & /\left(\operatorname{Imd~d~}_{\Pi_{\phi+n \chi / 2}}^{n} \cap \operatorname{Imd}_{\Pi_{\phi+(2 N-n) \chi / 2}}^{2 N-n}\right) \\
= & \left(\operatorname{Im~d}_{\Pi_{\phi+(2 N-n) \chi / 2}}^{2 N-n}+\operatorname{Im} \mathrm{I}_{\Pi_{\phi+n \chi / 2}}^{n}\right) / \operatorname{Im} \mathrm{d}_{\Pi_{\phi+n \chi / 2}}^{n},
\end{aligned}
$$

by standard homomorphism properties. Moreover,

$$
\begin{aligned}
\operatorname{Im~}_{\Pi_{\phi+n \chi / 2}}^{n+1} & \mathrm{~d}_{\Pi_{\phi+(2 N-n) \chi / 2}}^{2 N-n} \\
& =\mathrm{d}_{\Pi_{\phi+n \chi / 2}}^{n+1}\left(\operatorname{Im} \mathrm{d}_{\Pi_{\phi+n \chi / 2}}^{n}+\operatorname{Im~d}_{\Pi_{\phi+(2 N-n) \chi / 2}}^{2 N-n}\right)
\end{aligned}
$$

by the complex property and this equals

$$
\begin{aligned}
\left(\operatorname{Im~d}_{\Pi_{\phi+n \chi / 2}}^{n}\right. & \left.+\operatorname{Imd}_{\Pi_{\phi+(2 N-n) \chi / 2}}^{2 N-n}\right) / \operatorname{ker} \mathrm{d}_{\Pi_{\phi+n \chi / 2}}^{n+1} \\
& =\left(\operatorname{Imd}_{\Pi_{\phi+n \chi / 2}}^{n}+\operatorname{Im} \mathrm{d}_{\Pi_{\phi+(2 N-n) \chi / 2}}^{2 N-n}\right) / \operatorname{Imd}_{\Pi_{\phi+n \chi / 2}}^{n}
\end{aligned}
$$

by exactness. By Proposition 9.2, $\operatorname{Im}_{\Pi_{\phi+n \chi / 2}}^{n+1} \mathrm{~d}_{\Pi_{\phi+(2 N-n) \chi / 2}}^{2 N-n}$ is irreducible, hence so are all the above quotients, proving (2) and (3); (1) and (4) are proved similarly.

Theorem 9.2. The only non-zero submodules of $\mathcal{V}\left(\Pi, R\left(\pi_{N}\right)\right)$ are:

$$
\mathcal{V}\left(\Pi, R\left(\pi_{N}\right)\right) \supsetneq \operatorname{Im~d}_{\Pi_{\phi+N \chi / 2}}^{N} \supsetneq \operatorname{Im~d}_{\Pi_{\phi+(N+2) \chi / 2}}^{\mathrm{R}} .
$$

Proof. First of all,

$$
\operatorname{Imd}_{\Pi_{\phi+(N+2) \chi / 2}}^{\mathrm{R}}=\operatorname{ker} \mathrm{d}_{\Pi_{\phi+N \chi / 2}}^{N+1}, \quad \operatorname{ker} \mathrm{d}_{\Pi_{\phi+N \chi / 2}}^{\mathrm{R}}=\operatorname{Imd}_{\Pi_{\phi+N \chi / 2}}^{N},
$$

by exactness of the conformally symplectic pseudo de Rham complex. The submodule $\operatorname{Im} \mathrm{d}_{\Pi_{\phi+(N+2) \chi / 2}}^{\mathrm{R}}$ is irreducible by Corollary 9.1 and is therefore minimal in $\mathcal{V}\left(\Pi, R\left(\pi_{N}\right)\right)$. Similarly, $\mathrm{d}_{\Pi_{\phi+N \chi / 2}^{\mathrm{R}}} \mathcal{V}\left(\Pi, R\left(\pi_{N}\right)\right)$ is irreducible.

We now show that $\operatorname{Im}_{\Pi_{\phi+N \chi / 2}}^{N}$ is maximal in $\mathcal{V}\left(\Pi, R\left(\pi_{N}\right)\right)$. Indeed,

$$
\mathcal{V}\left(\Pi, R\left(\pi_{N}\right)\right) / \operatorname{Imd}_{\Pi_{\phi+N \chi / 2}}^{N}=\mathcal{V}\left(\Pi, R\left(\pi_{N}\right)\right) / \operatorname{ker} \mathrm{d}_{\Pi_{\phi+N \chi / 2}}^{\mathrm{R}} \simeq \operatorname{Imd}_{\Pi_{\phi+N \chi / 2}}^{\mathrm{R}},
$$

which is irreducible.

Finally, $\operatorname{Im}_{\Pi_{\phi+(N+2) \chi / 2}}^{\mathrm{R}}$ is a submodule of $\operatorname{Im~}_{\Pi_{\phi+N \chi / 2}}^{N}$, as $\mathrm{d}_{\Pi_{\phi+(N+2) \chi / 2}}^{\mathrm{R}}$ equals $\mathrm{d}_{\Pi_{\phi+N \chi / 2}}^{N} \mathrm{~d}_{\Pi_{\phi+(N+2) \chi / 2}^{N+1}}^{N+1}$ up to a non-zero multiplicative constant. It is indeed maximal, since

$$
\begin{aligned}
\operatorname{Im}_{\Pi_{\phi+N \chi / 2}}^{N} / & \operatorname{Im}_{\Pi_{\phi+(N+2) \chi / 2}}^{\mathrm{R}} \\
& \simeq \mathrm{d}_{\Pi_{\phi+N \chi / 2}^{N+1}}^{N+1} \operatorname{Im} \mathrm{d}_{\Pi_{\phi+N \chi / 2}}^{N} \\
& =\mathrm{d}_{\Pi_{\phi+(N-2) \chi / 2}^{N-1}}^{N-1} \mathrm{~d}_{\Pi_{\phi+(N+2) \chi / 2}}^{N+2} \mathcal{V}\left(\Pi_{\chi / 2}, R\left(\pi_{N-1}\right)\right)
\end{aligned}
$$


as $\operatorname{kerd}_{\Pi_{\phi+N \chi / 2}}^{N+1}=\operatorname{Imd}_{\Pi_{\phi+(N+2) \chi / 2}}^{\mathrm{R}}$. We have proved so far that the submodule $\operatorname{Im} \mathrm{d}_{\Pi_{\phi+N \chi / 2}}^{N}$ is maximal, that $\operatorname{Im~}_{\Pi_{\phi+(N+2) \chi / 2}^{R}}^{\mathrm{R}}=\operatorname{Im~}_{\Pi_{\phi+N \chi / 2}}^{N} \mathrm{~d}_{\Pi_{\phi+(N+2) \chi / 2}+1}^{N+1}$ is minimal, and that $\operatorname{Im} \mathrm{d}_{\Pi_{\phi+(N+2) \chi / 2}}^{\mathrm{R}}$ is maximal inside $\operatorname{Im} \mathrm{d}_{\Pi_{\phi+N \chi / 2}}^{N}$.

Every proper submodule contains non-constant homogeneous singular vectors, and is therefore contained in $\operatorname{Im~}_{\Pi_{\phi+N \chi / 2}}^{N}$. If it furthermore contains singular vectors of degree 1 , then it coincides with $\operatorname{Im~}_{\Pi_{\phi+N \chi / 2}}^{N}$, as this is generated by its singular vectors of degree 1 . If instead it does not contain singular vectors of degree 1 , then it lies between $\operatorname{Im} \mathrm{d}_{\Pi_{\phi+(N+2) \chi / 2}}^{\mathrm{R}}$ and $\operatorname{Im}_{\Pi_{\phi+N \chi / 2}}^{N}$, and must then coincide with the latter by maximality.

Lemma 9.4. The submodule

$$
\mathrm{d}_{\Pi_{\phi+(N-1) \chi / 2}}^{N-1} \mathcal{V}\left(\Pi_{\chi / 2}, R\left(\pi_{N-2}\right)\right)+\mathrm{d}_{\Pi_{\phi+(N+1) \chi / 2}}^{N+1} \mathcal{V}\left(\Pi_{\chi / 2}, R\left(\pi_{N}\right)\right) \subset \mathcal{V}\left(\Pi, R\left(\pi_{N-1}\right)\right)
$$

is maximal, and we have

$$
\begin{aligned}
\mathrm{d}_{\Pi_{\phi+(N-1) \chi / 2}}^{N-1} & \mathrm{~d}_{\Pi_{\phi+(N+3) \chi / 2}^{N+2}}^{N+1} \mathcal{V}\left(\Pi_{\chi}, R\left(\pi_{N-1}\right)\right) \\
& \left.=\mathrm{d}_{\Pi_{\phi+(N-1) \chi / 2}^{N-1}}^{N-1} \mathcal{V}\left(\Pi_{\chi / 2}, R\left(\pi_{N-2}\right)\right)\right) \cap \mathrm{d}_{\Pi_{\phi+(N+1) \chi / 2}+1}^{N+1} \mathcal{V}\left(\Pi_{\chi / 2}, R\left(\pi_{N}\right)\right)
\end{aligned}
$$

Proof. By Theorem 9.2, we know that the quotient

$$
\operatorname{Im}_{\Pi_{\phi+N \chi / 2}}^{N} / \mathrm{d}_{\Pi_{\phi+(N-1) \chi / 2}}^{N-1} \mathrm{~d}_{\Pi_{\phi+(N+3) \chi / 2}}^{N+2} \mathcal{V}\left(\Pi_{\chi}, R\left(\pi_{N-1}\right)\right)
$$

is irreducible. However, this equals

$$
\begin{aligned}
\operatorname{Imd}_{\Pi_{\phi+N \chi / 2}}^{N} / \mathrm{d}_{\Pi_{\phi+N \chi / 2}}^{N}\left(\operatorname{Im~d}_{\Pi_{\phi+(N-1) \chi / 2}}^{N-1}+\operatorname{Imd}_{\Pi_{\phi+(N+1) \chi / 2}}^{N+1}\right) \\
\quad=\mathcal{V}\left(\Pi_{\chi / 2}, R\left(\pi_{N-1}\right)\right) /\left(\operatorname{Imd}_{\Pi_{\phi+(N-1) \chi / 2}}^{N-1}+\operatorname{Im}_{\Pi_{\phi+(N+1) \chi / 2}}^{N+1}\right),
\end{aligned}
$$

thus proving the first claim.

Notice now that

$$
\operatorname{Im} \mathrm{d}_{\Pi_{\phi+(N-1) \chi / 2}}^{N-1} \mathrm{~d}_{\Pi_{\phi+(N+3) \chi / 2}+2}^{N+2}=\operatorname{Im~d}_{\Pi_{\phi+(N+1) \chi / 2}}^{N+1} \mathrm{~d}_{\Pi_{\phi+(N+1) \chi / 2}}^{N}
$$

is contained in $\operatorname{Im}_{\Pi_{\phi+(N-1) \chi / 2}}^{N-1} \cap \operatorname{Im~}_{\Pi_{\phi+(N+1) \chi / 2}}^{N+1}$, and that the quotient

$$
\left(\operatorname{Im} \mathrm{d}_{\Pi_{\phi+(N-1) \chi / 2}}^{N-1} \cap \operatorname{Im}_{\Pi_{\phi+(N+1) \chi / 2}}^{N+1}\right) / \operatorname{Im}_{\Pi_{\phi+(N-1) \chi / 2}}^{N-1} \mathrm{~d}_{\Pi_{\phi+(N+3) \chi / 2}}^{N+2}
$$

injects inside

$$
\begin{aligned}
\operatorname{Im~}_{\Pi_{\phi+(N+1) \chi / 2}}^{N+1} / \operatorname{Im~d}_{\Pi_{\phi+(N+1) \chi / 2}}^{N+1} \mathrm{~d}_{\Pi_{\phi+(N+1) \chi / 2}}^{N} & \\
= & \mathcal{V}\left(\Pi_{\chi / 2}, R\left(\pi_{N-2}\right)\right) /\left(\operatorname{Im~d}_{\Pi_{\phi+(N+1) \chi / 2}}^{N}+\operatorname{ker} \mathrm{d}_{\Pi_{\phi+(N+1) \chi / 2}}^{N+1}\right) \\
& =\mathcal{V}\left(\Pi_{\chi / 2}, R\left(\pi_{N-2}\right)\right) / \operatorname{Im~d}_{\Pi_{\phi+(N+1) \chi / 2}}^{N},
\end{aligned}
$$


which is irreducible. Therefore,

$$
\operatorname{Imd}_{\Pi_{\phi+(N-1) \chi / 2}}^{N-1} \cap \operatorname{Im}_{\Pi_{\phi+(N+1) \chi / 2}}^{N+1}=\operatorname{Imd}_{\Pi_{\phi+(N-1) \chi / 2}}^{N-1} \mathrm{~d}_{\Pi_{\phi+(N+3) \chi / 2}}^{N+2},
$$

since

$$
\operatorname{Imd}_{\Pi_{\phi+(N-1) \chi / 2}}^{N-1} \cap \operatorname{Im}_{\Pi_{\phi+(N+1) \chi / 2}}^{N+1} \neq \operatorname{Im}_{\Pi_{\phi+(N+1) \chi / 2}}^{N+1} .
$$

This completes the proof.

Proposition 9.4. If $1 \leq n<N$, then $\operatorname{Im}_{\Pi_{\phi+n \chi / 2}}^{n}+\operatorname{Im}_{\Pi_{\phi+(2 N-n) \chi / 2}}^{2 N-n}$ is maximal in $\mathcal{V}\left(\Pi, R\left(\pi_{n}\right)\right)$, and we have

$$
\operatorname{Im} \mathrm{d}_{\Pi_{\phi+n \chi / 2}}^{n} \mathrm{~d}_{\Pi_{\phi+(2 N-n+2) \chi / 2}}^{2 N-n+1}=\operatorname{Im~d}_{\Pi_{\phi+n \chi / 2}}^{n} \cap \operatorname{Im}_{\Pi_{\phi+(2 N-n) \chi / 2}}^{2 N-n} .
$$

Proof. The proof is by induction on $N-n$, the basis of induction being Lemma 9.4. The quotient

$$
\mathcal{V}\left(\Pi, R\left(\pi_{n}\right)\right) /\left(\operatorname{Im~d}_{\Pi_{\phi+n \chi / 2}}^{n}+\operatorname{Im~d}_{\Pi_{\phi+(2 N-n) \chi / 2}}^{2 N-n}\right)
$$

is isomorphic to

$$
\mathrm{d}_{\Pi_{\phi+n \chi / 2}}^{n+1} \mathcal{V}\left(\Pi, R\left(\pi_{n}\right)\right) / \operatorname{Im}_{\Pi_{\phi+n \chi / 2}}^{n+1} \mathrm{~d}_{\Pi_{\phi+(2 N-n) \chi / 2}}^{2 N-n},
$$

which equals

$$
\operatorname{Im} \mathrm{d}_{\Pi_{\phi+n \chi / 2}}^{n+1} /\left(\operatorname{Imd}_{\Pi_{\phi+n \chi / 2}}^{n+1} \cap \operatorname{Im~d}_{\Pi_{\phi+(2 N-n-2) \chi / 2}}^{2 N-n-1}\right)
$$

by the inductive hypothesis. However, this is irreducible by Proposition 9.3.

As for the second claim, notice that

$$
\operatorname{Im} \mathrm{d}_{\Pi_{\phi+n \chi / 2}}^{n} \mathrm{~d}_{\Pi_{\phi+(2 N-n+2) \chi / 2}}^{2 N-n+1} \subset \operatorname{Im}_{\Pi_{\phi+n \chi / 2}}^{n} \cap \operatorname{Im~}_{\Pi_{\phi+(2 N-n) \chi / 2}}^{2 N-n}
$$

is irreducible by Proposition 9.2, and is therefore minimal. Now proceed as in the proof of Lemma 9.4. The quotient

$$
\left(\operatorname{Im} \mathrm{d}_{\Pi_{\phi+n \chi / 2}}^{n} \cap \operatorname{Im}_{\Pi_{\phi+(2 N-n) \chi / 2}}^{2 N-n}\right) / \operatorname{Im}_{\Pi_{\phi+n \chi / 2}}^{n} \mathrm{~d}_{\Pi_{\phi+(2 N-n+2) \chi / 2}}^{2 N-n+1}
$$

injects inside

$$
\begin{aligned}
& \operatorname{Im}_{\Pi_{\phi+(2 N-n) \chi / 2}}^{2 N-n} / \operatorname{Im} \mathrm{d}_{\Pi_{\phi+(2 N-n) \chi / 2}}^{2 N-n} \mathrm{~d}_{\Pi_{\phi+(n+2) \chi / 2}}^{n+1} \\
& =\mathcal{V}\left(\Pi_{\chi / 2}, R\left(\pi_{n+1}\right)\right) /\left(\operatorname{Im~d}_{\Pi_{\phi+(n+2) \chi / 2}}^{n+1}+\operatorname{Im~d}_{\Pi_{\phi+(2 N-n) \chi / 2}}^{2 N-n-1}\right),
\end{aligned}
$$

which is irreducible by the inductive hypothesis. Now we claim 


$$
\operatorname{Im} \mathrm{d}_{\Pi_{\phi+n \chi / 2}}^{n} \cap \operatorname{Im}_{\Pi_{\phi+(2 N-n) \chi / 2}}^{2 N-n}=\operatorname{Im~d}_{\Pi_{\phi+n \chi / 2}}^{n} \mathrm{~d}_{\Pi_{\phi+(2 N-n+2) \chi / 2}}^{2 N-n+1} .
$$

Indeed, if we assume otherwise, then

$$
\operatorname{Im} \mathrm{d}_{\Pi_{\phi+n \chi / 2}}^{n} \cap \operatorname{Im}_{\Pi_{\phi+(2 N-n) \chi / 2}}^{2 N-n}=\operatorname{Imd}_{\Pi_{\phi+(2 N-n) \chi / 2}}^{2 N-n},
$$

whence

$$
\operatorname{Im} \mathrm{d}_{\Pi_{\phi+(2 N-n) \chi / 2}}^{2 N-n} \subset \operatorname{Im}_{\Pi_{\phi+n \chi / 2}}^{n}
$$

which is a contradiction.

Corollary 9.2. If $1 \leq n<N$, the intersection $\operatorname{Im~}_{\Pi_{\phi+n \chi / 2}}^{n} \cap \operatorname{Im}_{\Pi_{\phi+(2 N-n) \chi / 2}}^{2 N-n}$ is the only non-zero proper submodule of both $\operatorname{Im} \mathrm{d}_{\Pi_{\phi+n \chi / 2}}^{n}$ and $\operatorname{Im~}_{\Pi_{\phi+(2 N-n) \chi / 2}}^{2 N-n}$.

Proof. We have seen in Lemmas 9.1 and 9.2 that the only non-constant homogeneous singular vectors in both modules are contained in

$$
\left.\mathrm{d}_{\Pi_{\phi+n \chi / 2}}^{n} \mathrm{~d}_{\Pi_{\phi+(2 N-n+2) \chi / 2} N-n+1}^{2 N} \mathrm{~F}^{0} \mathcal{V}\left(\Pi_{\chi}, R\left(\pi_{n}\right)\right)\right),
$$

which $H$-generate $\operatorname{Im}_{\Pi_{\phi+n \chi / 2}}^{n} \mathrm{~d}_{\Pi_{\phi+(2 N-n+2) \chi / 2}}^{2 N-n+1}=\operatorname{Im}_{\Pi_{\phi+n \chi / 2}}^{n} \cap \operatorname{Imd}_{\Pi_{\phi+(2 N-n) \chi / 2}}^{2 N-n}$. Any proper submodule must then contain this submodule, which is however maximal in both $\operatorname{Im} \mathrm{d}_{\Pi_{\phi+n \chi / 2}}^{n}$ and $\operatorname{Im} \mathrm{d}_{\Pi_{\phi+(2 N-n) \chi / 2}}^{2 N-n}$, by Proposition 9.3.

Corollary 9.3. If $1 \leq n<N$ and $M$ is a proper submodule of $\mathcal{V}\left(\Pi, R\left(\pi_{n}\right)\right)$ strictly containing either $\operatorname{Im} \mathrm{d}_{\Pi_{\phi+n \chi / 2}}^{n}$ or $\operatorname{Im} \mathrm{d}_{\Pi_{\phi+(2 N-n) \chi / 2}}^{2 N-n}$, then

$$
M=\operatorname{Im}_{\Pi_{\phi+n \chi / 2}}^{n}+\operatorname{Im}_{\Pi_{\phi+(2 N-n) \chi / 2}}^{2 N-n}
$$

Proof. Let us treat the case $M \supsetneq \operatorname{Im~}_{\Pi_{\phi+(2 N-n) \chi / 2}}^{2 N-n}$, the other case being totally analogous. Submodules lying between $\operatorname{Imd}_{\Pi_{\phi+(2 N-n) \chi / 2}}^{2 N-n}$ and $\mathcal{V}\left(\Pi, R\left(\pi_{n}\right)\right)$ are in one-to-one correspondence with submodules of

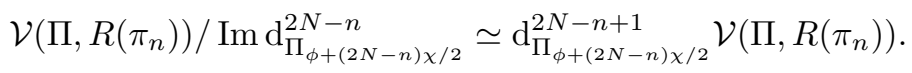

Thus, we may use Corollary 9.2 to show that there is a single possibility for $M$, which must be $\operatorname{Im} \mathrm{d}_{\Pi_{\phi+n \chi / 2}}^{n}+\operatorname{Imd}_{\Pi_{\phi+(2 N-n) \chi / 2}}^{2 N-n}$.

Proposition 9.5. If $1 \leq n<N$, a proper non-zero submodule of $\mathcal{V}\left(\Pi, R\left(\pi_{n}\right)\right)$ either contains or is contained in, either $\operatorname{Im} \mathrm{d}_{\Pi_{\phi+n \chi / 2}}^{n}$ or $\operatorname{Im~}_{\Pi_{\phi+(2 N-n) \chi / 2}}^{2 N-n}$.

Proof. Assume by contradiction that there is a submodule $M$ which neither contains nor is contained in the above two submodules; in particular, $M$ intersects both modules in 
$\operatorname{Im} \mathrm{d}_{\Pi_{\phi+n \chi / 2}}^{n} \mathrm{~d}_{\Pi_{\phi+(2 N-n+2) \chi / 2}}^{2 N-n+1}$. Then $M+\operatorname{Imd}_{\Pi_{\phi+n \chi / 2}}^{n}$ (respectively, $\left.M+\operatorname{Im~}_{\Pi_{\phi+(2 N-n) \chi / 2}}^{2 N-n}\right)$ strictly contains $\operatorname{Im} \mathrm{d}_{\Pi_{\phi+n \chi / 2}}^{n}$ (respectively, $\left.\operatorname{Im}_{\Pi_{\phi+(2 N-n) \chi / 2}}^{2 N-n}\right)$. However, the equalities

$$
M+\operatorname{Im~d}_{\Pi_{\phi+n \chi / 2}}^{n}=\mathcal{V}\left(\Pi, R\left(\pi_{n}\right)\right)=M+\operatorname{Im~d}_{\Pi_{\phi+(2 N-n) \chi / 2}}^{2 N-n}
$$

cannot both hold. If so, then

$$
\begin{aligned}
M / \operatorname{Im~d}_{\Pi_{\phi+n \chi / 2}}^{n} \mathrm{~d}_{\Pi_{\phi+(2 N-n+2) \chi / 2}}^{2 N-n+1} & =M /\left(M \cap \operatorname{Im}_{\Pi_{\phi+n \chi / 2}}^{n}\right) \\
& \simeq\left(M+\operatorname{Im} \mathrm{d}_{\Pi_{\phi+n \chi / 2}}^{n}\right) / \operatorname{Im~d}_{\Pi_{\phi+n \chi / 2}}^{n} \\
& \simeq \mathcal{V}\left(\Pi, R\left(\pi_{n}\right)\right) / \operatorname{Im~d}_{\Pi_{\phi+n \chi / 2}}^{n},
\end{aligned}
$$

and similarly

$$
\begin{aligned}
M / \operatorname{Im~}_{\Pi_{\phi+n \chi / 2}}^{n} \mathrm{~d}_{\Pi_{\phi+(2 N-n+2) \chi / 2}}^{2 N-n+1} & =M /\left(M \cap \operatorname{Imd}_{\Pi_{\phi+(2 N-n) \chi / 2}}^{2 N-n}\right) \\
& \simeq\left(M+\operatorname{Imd~}_{\Pi_{\phi+(2 N-n) \chi / 2}}^{2 N-n}\right) / \operatorname{Im~d}_{\Pi_{\phi+(2 N-n) \chi / 2}}^{2 N-n} \\
& \simeq \mathcal{V}\left(\Pi, R\left(\pi_{n}\right)\right) / \operatorname{Im~d}_{\Pi_{\phi+(2 N-n) \chi / 2}}^{2 N-n} .
\end{aligned}
$$

This would force

$$
\begin{aligned}
\mathrm{d}_{\Pi_{\phi+n \chi / 2}}^{n+1} \mathcal{V}\left(\Pi, R\left(\pi_{n}\right)\right) & \simeq \mathcal{V}\left(\Pi, R\left(\pi_{n}\right)\right) / \operatorname{Im}_{\Pi_{\phi+n \chi / 2}}^{n} \\
& \simeq \mathcal{V}\left(\Pi, R\left(\pi_{n}\right)\right) / \operatorname{Im~d}_{\Pi_{\phi+(2 N-n) \chi / 2}}^{2 N-n} \\
& \simeq \operatorname{Im~d}_{\Pi_{\phi+(2 N-n) \chi / 2}}^{2 N-n+1}
\end{aligned}
$$

which yields an easy contradiction by comparing the structure of singular vectors.

Let us thus assume that

$$
M+\operatorname{Im}_{\Pi_{\phi+n \chi / 2}}^{n}=\operatorname{Im~d}_{\Pi_{\phi+n \chi / 2}}^{n}+\operatorname{Imd}_{\Pi_{\phi+(2 N-n) \chi / 2}}^{2 N-n},
$$

as the other case is done similarly. Then

$$
\mathrm{d}_{\Pi_{\phi+n \chi / 2}}^{n+1} M=\operatorname{Im~d}_{\Pi_{\phi+n \chi / 2}}^{n+1} \mathrm{~d}_{\Pi_{\phi+(2 N-n) \chi / 2}}^{2 N-n} .
$$

However, ker $\mathrm{d}_{\Pi_{\phi+n \chi / 2}}^{n+1} \cap M$ is a submodule of $\operatorname{Imd}_{\Pi_{\phi+n \chi / 2}}^{n} \cap M$, which contains $\operatorname{Imd}_{\Pi_{\phi+n \chi / 2}}^{n} \cap \operatorname{Imd}_{\Pi_{\phi+(2 N-n) \chi / 2}^{2 N-n}}^{2 N}$. Since $M$ does not contain $\operatorname{Im}_{\Pi_{\phi+n \chi / 2}}^{n}$, it must equal $\operatorname{Im} \mathrm{d}_{\Pi_{\phi+n \chi / 2}}^{n} \cap \operatorname{Im} \mathrm{d}_{\Pi_{\phi+(2 N-n) \chi / 2}}^{2 N-n}$. Then

$$
\begin{aligned}
& M /\left(\operatorname{Im~d}_{\Pi_{\phi+n \chi / 2}}^{n} \cap \operatorname{Imd}_{\Pi_{\phi+(2 N-n) \chi / 2}}^{2 N-n}\right) \\
& \quad=M /\left(\operatorname{ker} \mathrm{d}_{\Pi_{\phi+n \chi / 2}}^{n+1} \cap M\right) \simeq \mathrm{d}_{\Pi_{\phi+n \chi / 2}}^{n+1} M=\operatorname{Im~}_{\Pi_{\phi+n \chi / 2}}^{n+1} \mathrm{~d}_{\Pi_{\phi+(2 N-n) \chi / 2}}^{2 N-n}
\end{aligned}
$$




$$
\begin{aligned}
& \simeq \operatorname{Imd}_{\Pi_{\phi+(2 N-n) \chi / 2}}^{2 N-n} /\left(\operatorname{ker} \mathrm{d}_{\Pi_{\phi+n \chi / 2}}^{n+1} \cap \operatorname{Imd}_{\Pi_{\phi+(2 N-n) \chi / 2}}^{2 N-n}\right) \\
& =\operatorname{Imd~d~}_{\Pi_{\phi+(2 N-n) \chi / 2}}^{2 N-n} /\left(\operatorname{Im~d}_{\Pi_{\phi+n \chi / 2}}^{n} \cap \operatorname{Im} \mathrm{d}_{\Pi_{\phi+(2 N-n) \chi / 2}}^{2 N-n}\right)
\end{aligned}
$$

thus showing $M=\operatorname{Im~d}_{\Pi_{\phi+(2 N-n) \chi / 2}}^{2 N-n}$, a contradiction.

The results of this section may be now summarized as follows:

Theorem 9.3. Let $V=\mathcal{V}(\Pi, U)$ be a tensor module over $H(\mathfrak{d}, \chi, \omega)$, where $\Pi$ and $U$ are finite-dimensional irreducible representations of $\mathfrak{d}$ and $\mathfrak{s p \mathfrak { d }}$, respectively. A complete list of $H(\mathfrak{d}, \chi, \omega)$-submodules of $V$ is as follows:

- $V \supset \operatorname{Im}_{\Pi_{\phi+N \chi}}^{2 N} \supset\{0\}$ if $U \simeq \mathbf{k}$;

- $V \supset \operatorname{Imd}_{\Pi_{\phi+n \times / 2}}^{n}+\operatorname{Imd}_{\Pi_{\phi+n}}^{2 N-n}$

$\operatorname{Im~d}_{\Pi_{\phi+n \chi / 2}}^{n} \cap \operatorname{Imd}_{\Pi_{\phi+(2 N-n) \chi / 2}}^{2 N-n} \supset\{0\}$ if $U \simeq R\left(\pi_{n}\right), 1 \leq n<N$;

- $V \supset \operatorname{Imd}_{\Pi_{\phi+N \chi / 2}^{N}}^{N} \supset \operatorname{Im~d}_{\Pi_{\phi+(N+2) \chi / 2}}^{\mathrm{R}} \supset\{0\}$ if $U=R\left(\pi_{N}\right)$;

- $V \supset\{0\}$ in all other cases.

\section{Submodules of tensor modules, non-graded case}

Throughout this section, we assume $\chi=0$ and $\omega=\mathrm{d} \zeta$, so that $\mathfrak{d}^{\prime}=\mathfrak{d}^{\zeta} \oplus \mathbf{k} c$ is a split central extension of $\mathfrak{d}$. Let $\Pi^{\prime}$ be a finite-dimensional irreducible $\mathfrak{d}^{\prime}$-module.

We have already seen that $\operatorname{sing} \mathcal{V}\left(\Pi^{\prime}, R\left(\pi_{n}\right)\right) \cap \mathrm{F}^{1} \mathcal{V}\left(\Pi^{\prime}, R\left(\pi_{n}\right)\right)$ contains canonical irreducible $\mathfrak{d}^{\prime} \oplus \mathfrak{s p} \mathfrak{d}$-summands isomorphic to $\Pi^{\prime} \otimes R\left(\pi_{n-1}\right)$ and $\Pi^{\prime} \otimes R\left(\pi_{n+1}\right)$. Recall that $\mathcal{V}\left(\Pi^{\prime}, R\left(\pi_{n}\right)\right)$ was constructed as an induced module, so that we may use its universal property in order to construct $H(\mathfrak{d}, 0, \mathrm{~d} \zeta)$-module homomorphisms

$$
\begin{aligned}
D_{\Pi^{\prime}}^{n}: \mathcal{V}\left(\Pi^{\prime}, R\left(\pi_{n-1}\right)\right) & \rightarrow \mathcal{V}\left(\Pi^{\prime}, R\left(\pi_{n}\right)\right), & & n=1, \ldots, N, \\
D_{\Pi^{\prime}}^{2 N-n}: \mathcal{V}\left(\Pi^{\prime}, R\left(\pi_{n+1}\right)\right) & \rightarrow \mathcal{V}\left(\Pi^{\prime}, R\left(\pi_{n}\right)\right), & & n=0, \ldots, N-1,
\end{aligned}
$$

which are, by Schur's Lemma, only determined up to multiplication by a nonzero scalar. Notice that when the $\mathfrak{d}^{\prime}$-module $\Pi^{\prime}$ factors through a $\mathfrak{d}$-module $\Pi$, i.e., when $c$ acts trivially and $\Pi^{\prime}=\pi^{*} \Pi$, then one has $D_{\Pi^{\prime}}^{n}=\mathrm{d}_{\Pi_{\phi}}^{n}$ and $D_{\Pi^{\prime}}^{2 N-n}=\mathrm{d}_{\Pi_{\phi}}^{2 N-n}$, up to the above undetermined scalar multiple. However, throughout the rest of this section, $c$ will act via multiplication by a nonzero scalar $\lambda \in \mathbf{k}$.

We also set $D_{\Pi^{\prime}}^{2 N+1}=D_{\Pi^{\prime}}^{0}=0$. Then $D_{\Pi^{\prime}}^{n+1} D_{\Pi^{\prime}}^{n}=0$ for all $0 \leq n \leq N-1$, as $\operatorname{sing} \mathcal{V}\left(\Pi^{\prime}, R\left(\pi_{n+1}\right)\right)$ contains no $\mathfrak{d}^{\prime} \oplus \mathfrak{s p} \mathfrak{d}$-irreducible summand isomorphic to $\Pi^{\prime} \otimes$ $R\left(\pi_{n-1}\right)$. Similarly, $D_{\Pi^{\prime}}^{2 N-n+1} D_{\Pi^{\prime}}^{2 N-n}=0$ for all $0 \leq n \leq N-1$. Recall that

$$
\begin{aligned}
& \operatorname{sing} \mathcal{V}\left(\Pi^{\prime}, R\left(\pi_{n}\right)\right) \cap \mathrm{F}^{1} \mathcal{V}\left(\Pi^{\prime}, R\left(\pi_{n}\right)\right) \\
& \quad=\mathrm{F}^{0} \mathcal{V}\left(\Pi^{\prime}, R\left(\pi_{n}\right)\right) \oplus D_{\Pi^{\prime}}^{n} \mathrm{~F}^{0} \mathcal{V}\left(\Pi^{\prime}, R\left(\pi_{n-1}\right)\right) \oplus D_{\Pi^{\prime}}^{2 N-n} \mathrm{~F}^{0} \mathcal{V}\left(\Pi^{\prime}, R\left(\pi_{n+1}\right)\right),
\end{aligned}
$$


when $1 \leq n \leq N-1$, whereas in the case $n=N$, the last direct summand is missing. Notice that when $1 \leq n \leq N, \operatorname{sing} \mathcal{V}\left(\Pi^{\prime}, R\left(\pi_{n}\right)\right)$ also contains an irreducible $\mathfrak{d}^{\prime} \oplus \mathfrak{s p} \mathfrak{d}$ summand isomorphic to $\Pi^{\prime} \otimes R\left(\pi_{n}\right)$ in degree two, which is however not canonically determined as any complement to $\mathrm{F}^{0} \mathcal{V}\left(\Pi^{\prime}, R\left(\pi_{n}\right)\right)$ in the $\Pi^{\prime} \otimes R\left(\pi_{n}\right)$-isotypical component of $\operatorname{sing} \mathcal{V}\left(\Pi^{\prime}, R\left(\pi_{n}\right)\right)$ will do. Recall also that, as $\lambda \neq 0$, we have no well-defined grading to choose a homogeneous canonical complement. However, we denote by $S_{\lambda}$ the space of singular vectors of degree 2 as constructed in Section 8.5.2 from homogeneous singular vectors.

Before computing the structure of singular vectors in the $H(\mathfrak{d}, 0, \mathrm{~d} \zeta)$-modules $\operatorname{Im} D_{\Pi^{\prime}}^{n}$ and $\operatorname{Im} D_{\Pi^{\prime}}^{2 N-n}$, we need some preliminary results.

Lemma 10.1. Let $M \subset \mathcal{V}\left(\Pi^{\prime}, U\right)$ be a proper $H(\mathfrak{d}, 0, \mathrm{~d} \zeta)$-submodule. If $M$ contains a vector $v \in \mathrm{F}^{1} \mathcal{V}\left(\Pi^{\prime}, U\right)$, then $v$ is singular.

Proof. We know by (6.14) that $\mathcal{P}_{2} \mathrm{~F}^{1} \mathcal{V}\left(\Pi^{\prime}, U\right)=0$. If $v$ is not singular, then $\mathcal{P}_{1} v$ contains a non-zero constant vector, which must lie in $M$. This yields a contradiction as proper submodules do not contain non-zero constant vectors, by Lemma 6.5.

Remark 10.1. The above lemma follows from the general fact that a vector of minimal degree in a proper submodule is always singular. However, we do not need this generality.

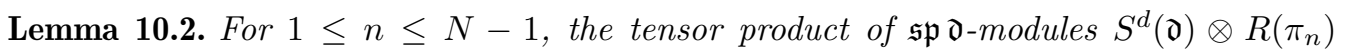
decomposes as

$$
\left.R\left(d \pi_{1}+\pi_{n}\right) \oplus R\left((d-1) \pi_{1}+\pi_{n-1}\right) \oplus R\left((d-1) \pi_{1}+\pi_{n+1}\right) \oplus R\left((d-2) \pi_{1}+\pi_{n}\right)\right) .
$$

Similarly, $S^{d}(\mathfrak{d}) \otimes R\left(\pi_{N}\right)$ decomposes as

$$
\left.R\left(d \pi_{1}+\pi_{N}\right) \oplus R\left((d-1) \pi_{1}+\pi_{N-1}\right) \oplus R\left((d-2) \pi_{1}+\pi_{N}\right)\right) .
$$

Proof. See, e.g., the reference tables at the end of [9].

Recall that the quotient $\mathcal{P}_{0} / \mathcal{P}_{1}$ acts on the space of singular vectors. However, the Lie subalgebra $\mathfrak{s p} \mathfrak{d} \simeq \mathfrak{p}_{0} \subset \mathcal{P}_{0}$ is a section of this quotient. It acts on each $H(\mathfrak{d}, 0, \mathrm{~d} \zeta)$-module and extends the $\mathfrak{s p} \mathfrak{d}$-action on singular vectors. As $\chi=0$, and we are disregarding the $\mathfrak{d}^{\prime}$-action, we have already seen that the $\mathfrak{s p} \mathfrak{d}$-action on $\operatorname{gr}^{d} \mathcal{V}\left(\Pi^{\prime}, U\right)$ is isomorphic to $\Pi^{\prime} \otimes\left(S^{d} \mathfrak{d} \otimes U\right)$, where we endow $\Pi^{\prime}$ with a trivial $\mathfrak{d}$-action. Every homomorphism of $H(\mathfrak{d}, 0, \mathrm{~d} \zeta)$-modules is also a $\mathfrak{p}_{0} \simeq \mathfrak{s p} \mathfrak{d}$-module homomorphism.

Proposition 10.1. Let $M \subset \mathcal{V}\left(\Pi^{\prime}, U\right)$ be a proper $H(\mathfrak{d}, 0, \mathrm{~d} \zeta)$-submodule. If $M$ contains a vector $v \in \mathrm{F}^{2} \mathcal{V}\left(\Pi^{\prime}, U\right)$ such that $v \equiv \psi(u) \bmod \mathrm{F}^{1} \mathcal{V}\left(\Pi^{\prime}, U\right)$, then $v$ is singular. 
Proof. We may assume without loss of generality that the action of $\mathfrak{s p d}$ on $U$ is nontrivial, so $U \simeq R\left(\pi_{n}\right)$ for some $1 \leq n \leq N$. The vector $v$ lies in $\mathrm{F}^{1} \mathcal{V}\left(\Pi^{\prime}, R\left(\pi_{n}\right)\right)+S_{\lambda}$, which is a $\mathfrak{p}_{0} \simeq \mathfrak{s p} \mathfrak{d}$-submodule of $\mathcal{V}\left(\Pi^{\prime}, R\left(\pi_{n}\right)\right)$ isomorphic to

$$
\Pi^{\prime} \otimes\left(R\left(\pi_{n}\right) \oplus\left(R\left(\pi_{n-1}\right) \oplus R\left(\pi_{n+1}\right) \oplus R\left(\pi_{1}+\pi_{n}\right)\right) \oplus R\left(\pi_{n}\right)\right)
$$

where we set $R\left(\pi_{N+1}\right)=0$ if $n=N$. As $M$ is a $\mathfrak{p}_{0}$-submodule of $\mathcal{V}\left(\Pi^{\prime}, R\left(\pi_{n}\right)\right)$, it must also contain the projection of $v$ to the $R\left(\pi_{1}+\pi_{n}\right)$-isotypical component, which only contains vectors of degree one that are non singular. By Lemma 10.2, this projection must vanish, hence $v$ lies in the sum of the other components, which only contain singular vectors.

Lemma 10.3. When $1 \leq n \leq N$, the $\mathfrak{s p d} \mathfrak{d}$-module $\operatorname{sing} \operatorname{Im} D_{\Pi^{\prime}}^{n}$ decomposes as the direct sum of $D_{\Pi^{\prime}}^{n} \mathrm{~F}^{0} \mathcal{V}\left(\Pi^{\prime}, R\left(\pi_{n-1}\right)\right)$ and an irreducible summand of singular vectors of degree 2.

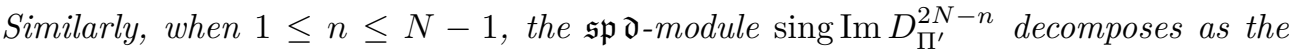
direct sum of $D_{\Pi^{\prime}}^{2 N-n} \mathrm{~F}^{0} \mathcal{V}\left(\Pi^{\prime}, R\left(\pi_{n+1}\right)\right)$ and an irreducible summand of singular vectors of degree 2 .

Proof. We will only prove the first statement, as the other one is completely analogous. The map $D_{\Pi^{\prime}}^{n}: \mathcal{V}\left(\Pi^{\prime}, R\left(\pi_{n-1}\right)\right) \rightarrow \mathcal{V}\left(\Pi^{\prime}, R\left(\pi_{n}\right)\right)$ is a homomorphism of $H(\mathfrak{d}, 0, \mathrm{~d} \zeta)$ modules; hence, it commutes with the action of $\mathfrak{p}_{0}$. We already know that

$$
\begin{aligned}
\operatorname{sing} \mathcal{V}\left(\Pi^{\prime}, R\left(\pi_{n}\right)\right)=\mathrm{F}^{0} \mathcal{V}\left(\Pi^{\prime}, R\left(\pi_{n}\right)\right) & \oplus D_{\Pi^{\prime}}^{n} \mathrm{~F}^{0} \mathcal{V}\left(\Pi^{\prime}, R\left(\pi_{n-1}\right)\right) \\
& \oplus D_{\Pi^{\prime}}^{2 N-n} \mathrm{~F}^{0} \mathcal{V}\left(\Pi^{\prime}, R\left(\pi_{n+1}\right)\right) \oplus S_{\lambda},
\end{aligned}
$$

where the first summand in the right-hand side is $\mathrm{F}^{0} \mathcal{V}\left(\Pi^{\prime}, R\left(\pi_{n}\right)\right)$, the next two summands are singular vectors of degree 1 , and the last summand consists of singular vectors of degree 2 as constructed in Section 8.5.2 starting from homogenous vectors.

The third summand does not lie in $\operatorname{Im} D_{\Pi^{\prime}}^{n}$, since by Lemma 10.2 the irreducible representation $R\left(\pi_{n+1}\right)$ does not show up as a direct summand in any $S^{d} \mathfrak{d} \otimes R\left(\pi_{n-1}\right)$. In particular, $\operatorname{Im} D_{\Pi^{\prime}}^{n}$ is a proper submodule of $\mathcal{V}\left(\Pi^{\prime}, R\left(\pi_{n}\right)\right)$, hence it does not contain constant vectors either. However, it contains $D_{\Pi^{\prime}}^{n} \mathrm{~F}^{0} \mathcal{V}\left(\Pi^{\prime}, R\left(\pi_{n-1}\right)\right)$, i.e., the second summand, by construction.

If $\Pi^{\prime} \otimes U$ is an irreducible representation of $\mathfrak{d}^{\prime} \oplus \mathfrak{s p \mathfrak { d }}$, and the action of $\mathfrak{s p} \mathfrak{d}$ is not trivial, we have seen in Corollary 7.1 that every submodule $M \subset \mathcal{V}\left(\Pi^{\prime}, U\right)$ contains a vector coinciding with $\psi(u)$ modulo $\mathrm{F}^{1} \mathcal{V}\left(\Pi^{\prime}, U\right)$, which must be singular due to Proposition 10.1. Thus $\operatorname{Im} D_{\Pi^{\prime}}^{n}$ contains an irreducible $\mathfrak{s p} \mathfrak{d}$-summand of degree two singular vectors.

Proposition 10.2. For each $1 \leq n \leq N-1$, the $H(\mathfrak{d}, 0, \mathrm{~d} \zeta)$-module $\mathcal{V}\left(\Pi^{\prime}, R\left(\pi_{n}\right)\right)$ decomposes as the direct sum of $\operatorname{Im} D_{\Pi^{\prime}}^{n}$ and $\operatorname{Im} D_{\Pi^{\prime}}^{2 N-n}$. Moreover, $\operatorname{Im} D_{\Pi^{\prime}}^{n}$ is an irreducible $H(\mathfrak{d}, 0, \mathrm{~d} \zeta)$-module for all $1 \leq n \leq N$. 
Proof. Let us treat the case $n=1$ first. We already know from Proposition 8.1 that $\mathcal{V}\left(\Pi^{\prime}, \mathbf{k}\right)$ is an irreducible $H(\mathfrak{d}, 0, \mathrm{~d} \zeta)$-module. As $D_{\Pi^{\prime}}^{1}$ is non-zero, it is injective by irreducibility of $\mathcal{V}\left(\Pi^{\prime}, \mathbf{k}\right)$, and its image is then isomorphic to $\mathcal{V}\left(\Pi^{\prime}, \mathbf{k}\right)$, hence $\operatorname{Im} D_{\Pi^{\prime}}^{1}$ is irreducible. The intersection $\operatorname{Im} D_{\Pi^{\prime}}^{1} \cap \operatorname{Im} D_{\Pi^{\prime}}^{2 N-1}$ is an $H(\mathfrak{d}, 0, \mathrm{~d} \zeta)$-submodule of $\operatorname{Im} D_{\Pi^{\prime}}^{1}$, which is irreducible, and comparing singular vectors shows that $\operatorname{Im} D_{\Pi^{\prime}}^{1}$ is not contained in $\operatorname{Im} D_{\Pi^{\prime}}^{2 N-1}$. Thus the intersection is trivial. As for the $\operatorname{sum} \operatorname{Im} D_{\Pi^{\prime}}^{1}+\operatorname{Im} D_{\Pi^{\prime}}^{2 N-1}$, it contains two irreducible summands of singular vectors of the degree 2 , which must be distinct as we already know the intersection to be trivial. Then $\operatorname{Im} D_{\Pi^{\prime}}^{1}+\operatorname{Im} D_{\Pi^{\prime}}^{2 N-1}$ contains constant vectors in $\mathcal{V}\left(\Pi^{\prime}, \mathbf{k}\right)$, hence it coincides with $\mathcal{V}\left(\Pi^{\prime}, \mathbf{k}\right)$.

Let us now proceed by induction on $n>1$. Assume we know that $\mathcal{V}\left(\Pi^{\prime}, R\left(\pi_{n}\right)\right)=$ $\operatorname{Im} D_{\Pi^{\prime}}^{n} \oplus \operatorname{Im} D_{\Pi^{\prime}}^{2 N-n}$. Then applying $D_{\Pi^{\prime}}^{n+1}$, we obtain

$$
\operatorname{Im} D_{\Pi^{\prime}}^{n+1}=D_{\Pi^{\prime}}^{n+1} \mathcal{V}\left(\Pi^{\prime}, R\left(\pi_{n}\right)\right)=D_{\Pi^{\prime}}^{n+1} D_{\Pi^{\prime}}^{2 N-n} \mathcal{V}\left(\Pi^{\prime}, R\left(\pi_{n+1}\right)\right)
$$

We know that each tensor module is generated by its constant singular vectors, and the same is true of the image of a tensor module via an $H(\mathfrak{d}, 0, \mathrm{~d} \zeta)$-homomorphism. Consequently, $\operatorname{Im} D_{\Pi^{\prime}}^{n+1}$ is generated over $H$ by $D_{\Pi^{\prime}}^{n+1} \mathrm{~F}^{0} \mathcal{V}\left(\Pi^{\prime}, R\left(\pi_{n}\right)\right)$, but also by $D_{\Pi^{\prime}}^{n+1} D_{\Pi^{\prime}}^{2 N-n} \mathrm{~F}^{0} \mathcal{V}\left(\Pi^{\prime}, R\left(\pi_{n+1}\right)\right)$, as they are the two $\mathfrak{d}^{\prime} \oplus \mathfrak{s p} \mathfrak{d}$-irreducible summands of sing $\operatorname{Im} D_{\Pi^{\prime}}^{n+1}$. As every $H(\mathfrak{d}, 0, \mathrm{~d} \zeta)$-submodule of $\operatorname{Im} D_{\Pi^{\prime}}^{n+1}$ must contain non-zero singular vectors, this shows that $\operatorname{Im} D_{\Pi^{\prime}}^{n+1}$ has no proper non-zero submodules, i.e., it is irreducible.

Once we know that $\operatorname{Im} D_{\Pi^{\prime}}^{n+1}$ is $H(\mathfrak{d}, 0, \mathrm{~d} \zeta)$-irreducible, and $n+1 \neq N$, then we may argue as in the case $n=1$ that $\operatorname{Im} D_{\Pi^{\prime}}^{n+1} \cap \operatorname{Im} D_{\Pi^{\prime}}^{2 N-n-1}=\{0\}$ by irreducibility of $\operatorname{Im} D_{\Pi^{\prime}}^{n+1}$ and $\mathcal{V}\left(\Pi^{\prime}, R\left(\pi_{n+1}\right)\right)=\operatorname{Im} D_{\Pi^{\prime}}^{n+1}+\operatorname{Im} D_{\Pi^{\prime}}^{2 N-n-1}$ because it contains non-zero constant vectors. In other words, $\mathcal{V}\left(\Pi^{\prime}, R\left(\pi_{n+1}\right)\right)=\operatorname{Im} D_{\Pi^{\prime}}^{n+1} \oplus \operatorname{Im} D_{\Pi^{\prime}}^{2 N-n-1}$, as claimed.

Proposition 10.3. For each $0 \leq n \leq N-2$, the $H(\mathfrak{d}, 0, \mathrm{~d} \zeta)$-module $\operatorname{Im} D_{\Pi^{\prime}}^{2 N-n}$ is irreducible.

Proof. The case $n=0$ is immediate, as $\operatorname{Im} D_{\Pi^{\prime}}^{2 N}=\mathcal{V}(\Pi, \mathbf{k})$. If $n>0$, we know that

$$
\operatorname{Im} D_{\Pi^{\prime}}^{2 N-n}=D_{\Pi^{\prime}}^{2 N-n} \mathcal{V}\left(\Pi^{\prime}, R\left(\pi_{n+1}\right)\right)=D_{\Pi^{\prime}}^{2 N-n}\left(\operatorname{Im} D_{\Pi^{\prime}}^{n+1} \oplus \operatorname{Im} D_{\Pi^{\prime}}^{2 N-n-1}\right) .
$$

Thus,

$$
\operatorname{Im} D_{\Pi^{\prime}}^{2 N-n}=D_{\Pi^{\prime}}^{2 N-n} \mathcal{V}\left(\Pi^{\prime}, R\left(\pi_{n+1}\right)\right)=D_{\Pi^{\prime}}^{2 N-n} D_{\Pi^{\prime}}^{n+1} \mathcal{V}\left(\Pi^{\prime}, R\left(\pi_{n}\right)\right)
$$

Then we may argue, as in the proof of Proposition 10.2, that $\operatorname{Im} D_{\Pi^{\prime}}^{2 N-n}$ is generated over $H$ by each of the two $\mathfrak{d}^{\prime} \oplus \mathfrak{s p} \mathfrak{d}$-irreducible summands of singular vectors it contains. Hence, it is irreducible.

We have seen above that $\operatorname{Im} D_{\Pi^{\prime}}^{N} \subset \mathcal{V}\left(\Pi^{\prime}, R\left(\pi_{N}\right)\right)$ is irreducible, and we know that its singular vectors contain a degree two summand. Let 


$$
\eta: \mathcal{V}\left(\Pi^{\prime}, R\left(\pi_{N}\right)\right) \rightarrow \mathcal{V}\left(\Pi^{\prime}, R\left(\pi_{N}\right)\right)
$$

be the $H(\mathfrak{d}, 0, \mathrm{~d} \zeta)$-module homomorphism that maps non-trivially $\mathrm{F}^{0} \mathcal{V}\left(\Pi^{\prime}, R\left(\pi_{N}\right)\right)$ to this summand. Then $\operatorname{Im} \eta$ is contained in $\operatorname{Im} D_{\Pi^{\prime}}^{N}$, and coincides with it by irreducibility of $\operatorname{Im} D_{\Pi^{\prime}}^{N}$. In particular, $\eta$ is not injective, as it is not injective on $\operatorname{sing} \mathcal{V}\left(\Pi^{\prime}, R\left(\pi_{N}\right)\right)$. Then $\operatorname{ker} \eta$ is a proper $H(\mathfrak{d}, 0, \mathrm{~d} \zeta)$-submodule of $\mathcal{V}\left(\Pi^{\prime}, R\left(\pi_{N}\right)\right)$, which cannot contain constant vectors.

Lemma 10.4. The $H(\mathfrak{d}, 0, \mathrm{~d} \zeta)$-submodule $\operatorname{ker} \eta \subset \mathcal{V}\left(\Pi^{\prime}, R\left(\pi_{N}\right)\right)$ does not contain singular vectors of degree 1 .

Proof. As $\eta$ is an $H(\mathfrak{d}, 0, \mathrm{~d} \zeta)$-homomorphism, it commutes with the action of $\mathfrak{p}_{0} \simeq \mathfrak{s p} \mathfrak{d}$, which provides a section of $\mathcal{P}_{0} / \mathcal{P}_{1}$ in $\mathcal{P}_{0}$. We have seen that $\operatorname{gr}^{d} \mathcal{V}\left(\Pi^{\prime}, R\left(\pi_{N}\right)\right) \simeq \Pi^{\prime} \otimes$ $\left(S^{d}(\mathfrak{d}) \otimes R\left(\pi_{N}\right)\right)$ as $\mathfrak{s p} \mathfrak{d}$-modules, where $\Pi^{\prime}$ is considered as trivial. However,

$$
S^{d}(\mathfrak{d}) \otimes R\left(\pi_{N}\right) \simeq R\left(d \pi_{1}+\pi_{N}\right) \oplus R\left((d-1) \pi_{1}+\pi_{N-1}\right) \oplus R\left((d-2) \pi_{1}+\pi_{N}\right)
$$

contains an irreducible summand isomorphic to $R\left(\pi_{N-1}\right)$ only when $d=1$, and this summand corresponds to singular vectors of degree 1 in $\mathcal{V}\left(\Pi^{\prime}, R\left(\pi_{N}\right)\right)$. We know that singular vectors of degree 1 in $\mathcal{V}\left(\Pi^{\prime}, R\left(\pi_{N}\right)\right)$ lie in the image of $\eta$; however, they can only be the image via $\eta$ of singular vectors of degree 1 , which therefore do not lie in ker $\eta$.

As $\operatorname{ker} \eta$ is a proper submodule of $\mathcal{V}\left(\Pi^{\prime}, R\left(\pi_{N}\right)\right)$, it must contain non-trivial singular vectors, that can only have degree 2 . We will denote by $D_{\Pi^{\prime}}^{R}$ the $H(\mathfrak{d}, 0, \mathrm{~d} \zeta)$ endomorphism of $\mathcal{V}\left(\Pi^{\prime}, R\left(\pi_{N}\right)\right)$ that maps constant singular vectors to the summand of degree 2 singular vectors contained in ker $\eta$. Notice that when $\lambda=0, D_{\Pi^{\prime}}^{R}$ coincides with $\mathrm{d}_{\Pi_{\phi}}^{\mathrm{R}}$, as usual up to a nonzero multiplicative constant.

Proposition 10.4. The image of $D_{\Pi^{\prime}}^{R}: \mathcal{V}\left(\Pi^{\prime}, R\left(\pi_{N}\right)\right) \rightarrow \mathcal{V}\left(\Pi^{\prime}, R\left(\pi_{N}\right)\right)$ is irreducible. Moreover,

(1) $\mathcal{V}\left(\Pi^{\prime}, R\left(\pi_{N}\right)\right)=\operatorname{Im} D_{\Pi^{\prime}}^{R} \oplus \operatorname{Im} D_{\Pi^{\prime}}^{N}$;

(2) $\operatorname{ker} D_{\Pi^{\prime}}^{R}=\operatorname{Im} D_{\Pi^{\prime}}^{N}$;

(3) $\operatorname{Im} D_{\Pi^{\prime}}^{N} \simeq \operatorname{Im} D_{\Pi^{\prime}}^{N+1}$;

(4) $\operatorname{Im} D_{\Pi^{\prime}}^{N+1}$ is an irreducible $H(\mathfrak{d}, 0, \mathrm{~d} \zeta)$-module.

Proof. The image of $D_{\Pi^{\prime}}^{R}$ is contained in ker $\eta$, so also $\operatorname{sing} \operatorname{Im} D_{\Pi^{\prime}}^{R} \subset \operatorname{sing} \operatorname{ker} \eta$. As sing ker $\eta$ is $\mathfrak{d}^{\prime} \oplus \mathfrak{s p} \mathfrak{d}$-irreducible, the same holds for $\operatorname{sing} \operatorname{Im} D_{\Pi^{\prime}}^{R}$. However, $\operatorname{Im} D_{\Pi^{\prime}}^{R}=$ $D_{\Pi^{\prime}}^{R} \mathcal{V}\left(\Pi^{\prime}, R\left(\pi_{N}\right)\right)$ is spanned over $H$ by $D_{\Pi^{\prime}}^{R} \mathrm{~F}^{0} \mathcal{V}\left(\Pi^{\prime}, R\left(\pi_{N}\right)\right)$ so it is certainly irreducible, as any submodule must contain non-trivial singular vectors, hence all of $D_{\Pi^{\prime}}^{R} \mathrm{~F}^{0} \mathcal{V}\left(\Pi^{\prime}, R\left(\pi_{N}\right)\right)$. Let us take care of the remaining claims. 
(1) The direct sum decomposition clearly holds once we know that the intersection is trivial, as the sum must then contain non-zero constant vectors. However, as sing $\operatorname{Im} D_{\Pi^{\prime}}^{R}$ does not contain singular vectors of degree one, $\operatorname{Im} D_{\Pi^{\prime}}^{R} \cap \operatorname{Im} D_{\Pi^{\prime}}^{N} \neq 0$ would imply $\operatorname{Im} D_{\Pi^{\prime}}^{R} \subsetneq \operatorname{Im} D_{\Pi^{\prime}}^{N}$, which is impossible as $\operatorname{Im} D_{\Pi^{\prime}}^{N}$ is irreducible.

(2) $\operatorname{As} \operatorname{Im} D_{\Pi^{\prime}}^{N}$ and $\operatorname{Im} D_{\Pi^{\prime}}^{R}$ are non-isomorphic irreducible $H(\mathfrak{d}, 0, \mathrm{~d} \zeta)$-modules, and the image of $D_{\Pi^{\prime}}^{R}$ is (tautologically) isomorphic to $\operatorname{Im} D_{\Pi^{\prime}}^{R}$, then $D_{\Pi^{\prime}}^{R}$ must map $\operatorname{Im} D_{\Pi^{\prime}}^{N}$ to 0 .

(3) Since $\mathcal{V}\left(\Pi^{\prime}, R\left(\pi_{N}\right)\right)=\operatorname{Im} D_{\Pi^{\prime}}^{R} \oplus \operatorname{Im} D_{\Pi^{\prime}}^{N}$ is the direct sum of two irreducible summands, the non-zero image of $D_{\Pi^{\prime}}^{N+1}: \mathcal{V}\left(\Pi^{\prime}, R\left(\pi_{N}\right)\right) \rightarrow \mathcal{V}\left(\Pi^{\prime}, R\left(\pi_{N-1}\right)\right)$ is isomorphic to either one of the summands or to their sum. However, we know that $\operatorname{sing} \operatorname{Im} D_{\Pi^{\prime}}^{N+1}$ has exactly two $\mathfrak{d}^{\prime} \oplus \mathfrak{s p} \mathfrak{d}$-irreducible summands, therefore $\operatorname{Im} D_{\Pi^{\prime}}^{N+1}$ is necessarily isomorphic to $\operatorname{Im} D_{\Pi^{\prime}}^{N}$

(4) Follows from irreducibility of $\operatorname{Im} D_{\Pi^{\prime}}^{N}$.

We are ready to summarize the results of this section in the following statement.

Theorem 10.1. Let $\Pi^{\prime}$ denote a finite-dimensional irreducible $\mathfrak{d}^{\prime}$-module with a non-trivial action of $c \in \mathfrak{d}^{\prime}$. Then the complex of $H(\mathfrak{d}, 0, \mathrm{~d} \zeta)$-modules

$$
\begin{aligned}
0 & \rightarrow \mathcal{V}\left(\Pi^{\prime}, R\left(\pi_{0}\right)\right) \stackrel{D_{\Pi^{\prime}}^{1}}{\longrightarrow} \mathcal{V}\left(\Pi^{\prime}, R\left(\pi_{1}\right)\right) \stackrel{D_{\Pi^{\prime}}^{2}}{\longrightarrow} \cdots \stackrel{D_{\Pi^{\prime}}^{N}}{\longrightarrow} \mathcal{V}\left(\Pi^{\prime}, R\left(\pi_{N}\right)\right) \\
& \stackrel{D_{\Pi^{\prime}}^{R}}{\longrightarrow} \mathcal{V}\left(\Pi^{\prime}, R\left(\pi_{N}\right)\right) \stackrel{D_{\Pi^{\prime}}^{N+1}}{\longrightarrow} \mathcal{V}\left(\Pi^{\prime}, R\left(\pi_{N-1}\right)\right) \\
& \stackrel{D_{\Pi^{\prime}}^{N+2}}{\longrightarrow} \cdots \stackrel{D_{\Pi^{\prime}}^{2 N}}{\longrightarrow} \mathcal{V}\left(\Pi^{\prime}, R\left(\pi_{0}\right)\right) \rightarrow 0,
\end{aligned}
$$

is exact and $\operatorname{Im} D_{\Pi^{\prime}}^{2 N-n} \simeq \operatorname{Im} D_{\Pi^{\prime}}^{n+1}$ is irreducible for all $n$, as well as $\operatorname{Im} D_{\Pi^{\prime}}^{R}$. One has

$$
\begin{aligned}
\mathcal{V}\left(\Pi^{\prime}, R\left(\pi_{n}\right)\right) & =\operatorname{Im} D_{\Pi^{\prime}}^{n} \oplus \operatorname{Im} D_{\Pi^{\prime}}^{2 N-n}, \quad 0 \leq n \leq N-1, \\
\mathcal{V}\left(\Pi^{\prime}, R\left(\pi_{N}\right)\right) & =\operatorname{Im} D_{\Pi^{\prime}}^{N} \oplus \operatorname{Im} D_{\Pi^{\prime}}^{R} .
\end{aligned}
$$

Proof. Exactness whenever $D_{\Pi^{\prime}}^{R}$ is not involved follows from the fact that $\operatorname{Im} D_{\Pi^{\prime}}^{n+1}$ is irreducible, hence $\operatorname{ker} D_{\Pi^{\prime}}^{n}$ is maximal and contains $\operatorname{Im} D_{\Pi^{\prime}}^{n}$. The isomorphism $\operatorname{Im} D_{\Pi^{\prime}}^{2 N-n} \simeq$ $\operatorname{Im} D_{\Pi^{\prime}}^{n+1}$ follows from the fact that $D_{\Pi^{\prime}}^{n+1}$ acts trivially on $\operatorname{Im} D_{\Pi^{\prime}}^{n}$ and faithfully on $\operatorname{Im} D_{\Pi^{\prime}}^{2 N-n}$. Everything else has already been proved.

Remark 10.2. We see now that the map $D_{\Pi^{\prime}}^{R}$ coincides with $\left(D_{\Pi^{\prime}}^{R}\right)^{2}$ up to a nonzero multiplicative factor, and we may normalize it so that $\left(D_{\Pi^{\prime}}^{R}\right)^{2}=D_{\Pi^{\prime}}^{R}$. Then $\mathrm{Id}-D_{\Pi^{\prime}}^{R}$ is the projector on $\operatorname{Im} D_{\Pi^{\prime}}^{N}$ and thus may be identified with $\eta$, up to scalar multiples. With a similar argument, one may also identify $\eta$ with $D_{\Pi^{\prime}}^{N} D_{\Pi^{\prime}}^{N+1}$. 


\section{Classification of finite irreducible modules over $H(\mathfrak{d}, \chi, \omega)$}

We can now complete the classification of finite irreducible modules over the Lie pseudoalgebra $H(\mathfrak{d}, \chi, \omega)$.

Theorem 11.1. A complete list of non-trivial pairwise non-isomorphic irreducible finite $H(\mathfrak{d}, \chi, \omega)$-modules is as follows:

- tensor modules $\mathcal{V}\left(\Pi^{\prime}, U\right)$ where $\Pi^{\prime} \otimes U$ is a finite-dimensional irreducible $\mathfrak{d}^{\prime} \oplus \mathfrak{s p \mathfrak { d } -}$ module and $U$ is not isomorphic to any $R\left(\pi_{n}\right), 0 \leq n \leq N$;

- $\operatorname{Im} \mathrm{d}_{\Pi_{\phi+n \chi / 2}}^{n} \mathrm{~d}_{\Pi_{\phi+(2 N-n+2) \chi / 2}}^{2 N-n+1}, 1 \leq n \leq N$, where $\Pi$ is a finite-dimensional irreducible d-module;

- $\operatorname{Im} D_{\Pi^{\prime}}^{n}, 1 \leq n \leq N$, where $\Pi^{\prime}$ is a finite-dimensional irreducible $\mathfrak{d}^{\prime}$-module with a non-trivial action of $c \in \mathfrak{d}^{\prime}$;

- $\operatorname{Im} D_{\Pi^{\prime}}^{R}$, where $\Pi^{\prime}$ is a finite-dimensional irreducible $\mathfrak{d}^{\prime}$-module with a non-trivial action of $c \in \mathfrak{d}^{\prime}$.

Proof. Due to Theorem 6.1, irreducible $H(\mathfrak{d}, \chi, \omega)$-modules are all obtained as quotients of a tensor module $\mathcal{V}\left(\Pi^{\prime}, U\right)$ by a maximal submodule, where $\Pi^{\prime} \otimes U$ is a finite-dimensional irreducible $\mathfrak{d}^{\prime} \oplus \mathfrak{s p} \mathfrak{d}$-module. If $U$ is not isomorphic to any $R\left(\pi_{n}\right), 0 \leq n \leq N$, then Theorem 7.1 shows that $\mathcal{V}\left(\Pi^{\prime}, U\right)$ is itself irreducible. In all other cases, the maximal submodules of $\mathcal{V}\left(\Pi^{\prime}, U\right)$ are determined in Sections 9 and 10.

When $c$ acts non-trivially on $\Pi^{\prime}$, the description of irreducible quotients of $\mathcal{V}\left(\Pi^{\prime}, U\right)$ follows from Theorem 10.1. Suppose now that $c$ acts trivially on $\Pi^{\prime}$; then $\Pi^{\prime}=\pi^{*} \Pi$ for some $\mathfrak{d}$-module $\Pi$, where $\pi: \mathfrak{d}^{\prime} \rightarrow \mathfrak{d}^{\prime} / \mathbf{k} c \simeq \mathfrak{d}$ denotes the canonical projection. Henceforth, we will use the notation introduced in Definition 6.2(iii).

By Theorem 9.3, the unique maximal submodule of $\mathcal{V}\left(\Pi, R\left(\pi_{n}\right)\right)$, for $1 \leq n<N$, is $M=\operatorname{Imd}_{\Pi_{\phi+n \chi / 2}}^{n}+\operatorname{Imd}_{\Pi_{\phi+(2 N-n) \chi / 2}}^{2 N-n}$, which clearly lies in the kernel of the homomorphisms

$$
\mathrm{d}_{\Pi_{\phi+n \chi / 2}}^{n} \mathrm{~d}_{\Pi_{\phi+(2 N-n+2) \chi / 2}}^{2 N-n+1}, \quad \mathrm{~d}_{\Pi_{\phi+(2 N-n) \chi / 2}}^{2 N-n} \mathrm{~d}_{\Pi_{\phi+(n+2) \chi / 2}}^{n+1},
$$

which both map $\mathcal{V}\left(\Pi_{\chi}, R\left(\pi_{n}\right)\right)$ to $\mathcal{V}\left(\Pi, R\left(\pi_{n}\right)\right)$ and coincide up to multiplication by a non-zero scalar. As the above maps are non-zero, their kernel must be equal to $M$; hence

$$
\operatorname{Im~d}_{\Pi_{\phi+n \chi / 2}}^{n} \mathrm{~d}_{\Pi_{\phi+(2 N-n+2) \chi / 2}}^{2 N-n+1} \simeq \mathcal{V}\left(\Pi, R\left(\pi_{n}\right)\right) / M
$$

is the unique irreducible quotient of $\mathcal{V}\left(\Pi, R\left(\pi_{n}\right)\right)$.

As for $\mathcal{V}\left(\Pi, R\left(\pi_{N}\right)\right)$, its unique maximal submodule is $M=\operatorname{Im~}_{\Pi_{\phi+N \chi / 2}}^{N}$, which lies in the kernel of the non-zero map $\mathrm{d}_{\Pi_{\phi+(N+2) \chi / 2}^{\mathrm{R}}}: \mathcal{V}\left(\Pi, R\left(\pi_{N}\right)\right) \rightarrow \mathcal{V}\left(\Pi_{-\chi}, R\left(\pi_{N}\right)\right)$. Thus,

$$
\operatorname{Im}_{\Pi_{\phi+(N+2) \chi / 2}}^{\mathrm{R}} \simeq \mathcal{V}\left(\Pi, R\left(\pi_{N}\right)\right) / M
$$


is the unique irreducible quotient of $\mathcal{V}\left(\Pi, R\left(\pi_{N}\right)\right)$.

Finally, the only proper non-zero submodule of $\mathcal{V}(\Pi, \mathbf{k})$ is $\operatorname{Im}_{\Pi_{\phi+N \chi}}^{2 N}$, which is of finite $\mathbf{k}$-codimension. Its $\mathfrak{d} \oplus \mathfrak{s p} \mathfrak{d}$-irreducible quotient is thus a torsion $H$-module, which is thence endowed with a trivial action.

As representations of the Lie pseudoalgebra $H(\mathfrak{d}, \chi, \omega)$ are in one-to-one correspondence with conformal (in the sense of the definition given in Section 2.4) representations of its extended annihilation algebra $\mathfrak{d} \ltimes \mathcal{P}$, one may use the above results to deduce a classification of irreducible (topological, discrete) $P_{2 N}$-modules and of singular vectors in Verma modules induced from irreducible $\mathfrak{s p}_{2 N}$ representations as follows.

Let $\mathfrak{d}$ be a $2 N$-dimensional Lie algebra endowed with a Frobenius structure $\omega=\mathrm{d} \zeta$; for instance, one may choose $\mathfrak{d}$ to be the direct sum of $N$ copies of the non-abelian 2-dimensional Lie algebra. The extended annihilation algebra $\widetilde{\mathcal{P}}=\mathfrak{d} \ltimes \mathcal{P}$ of $H(\mathfrak{d}, 0, \mathrm{~d} \zeta)$ decomposes as a direct sum of Lie algebras $\widetilde{\mathcal{P}}=\mathfrak{d}^{\zeta} \oplus \mathcal{P}$. Indeed, as $\chi=0$, elements $\widetilde{\partial}$ act on $\mathcal{P}$ by inner derivations, and as $\omega=\mathrm{d} \zeta$, the central extension yielding $\mathfrak{d}^{\prime}$ may be trivialized to $\mathfrak{d}^{\prime}=\mathfrak{d}^{\zeta} \oplus \mathbf{k} c$. Let $U$ be a representation of $\mathfrak{s p} \mathfrak{d}$. Using $\mathfrak{s p} \mathfrak{d} \simeq \mathcal{P}_{0} / \mathcal{P}_{1}$, we endow $U$ with an action of $\mathcal{P}_{0}$ and extend it to $\mathcal{N}_{\mathcal{P}}$ by letting $\mathfrak{d}^{\zeta}$ act trivially on $U$ and $c$ act via multiplication by a scalar $\lambda \in \mathbf{k}$. Then the induced module $\operatorname{Ind}_{\mathcal{N}_{\mathcal{P}}}^{\widetilde{P}}$ is isomorphic to the tensor module $\mathcal{V}\left(\mathbf{k}_{\lambda}, U\right)$, where $\mathbf{k}_{\lambda}$ denotes the 1-dimensional $\mathfrak{d}^{\prime}$-module where $c$ acts as $\lambda$ and $\mathfrak{d}^{\zeta}$ acts trivially. Notice that elements in $\mathfrak{d}^{\zeta}$ act trivially on all of $\mathcal{V}\left(\mathbf{k}_{\lambda}, U\right)$, so that the submodule lattice of $\mathcal{V}\left(\mathbf{k}_{\lambda}, U\right)$ as an $H(\mathfrak{d}, 0, \mathrm{~d} \zeta)$-module only depends on its structure as a representation of $\mathcal{P}$. Also, when $\lambda=0$, the action of $\mathcal{P}$ factors through its quotient $\mathcal{H}$.

We thus recover an old result of Rudakov as claim $(i)$ and $(i i)$ of the following proposition.

Proposition 11.1 ([10]).

(i) Every non-constant homogeneous singular vector in the $\mathcal{H}$-module $V=\mathcal{V}(\mathbf{k}, U)$ has degree 1 or 2 . The space $S$ of such singular vectors is an $\mathfrak{s p d - m o d u l e ~ a n d ~ t h e ~}$ quotient of $V$ by the $\mathcal{H}$-submodule generated by $S$ is an irreducible $\mathcal{H}$-module. All singular vectors of degree 1 are listed in cases (a), (b) below, while all singular vectors of degree 2 are listed in case (c):

(a) $U=R\left(\pi_{p}\right), \quad S=R\left(\pi_{p+1}\right), \quad 0 \leq p \leq N-1$.

(b) $U=R\left(\pi_{p}\right), \quad S=R\left(\pi_{p-1}\right), \quad 1 \leq p \leq N$.

(c) $U=R\left(\pi_{p}\right), \quad S=R\left(\pi_{p}\right), \quad 1 \leq p \leq N$.

(ii) If the $\mathfrak{s p d}$-module $U$ is infinite-dimensional irreducible, then $\mathcal{V}(\mathbf{k}, U)$ does not contain non-constant singular vectors.

(iii) If an $\mathcal{H}$-module $\mathcal{V}(\mathbf{k}, U)$ is not irreducible, then its unique irreducible quotient is isomorphic to the topological dual of the kernel of the differential of a member of the Eastwood complex from [6] over formal power series. 
The next proposition deals with conformal representations of $\mathcal{P} \simeq P_{2 N}$ with a nontrivial action of the center.

Proposition 11.2. Let $0 \neq \lambda \in \mathbf{k}$.

(i) Every non-constant homogeneous singular vector in the $\mathcal{P}$-module $V=\mathcal{V}\left(\mathbf{k}_{\lambda}, U\right)$ lies

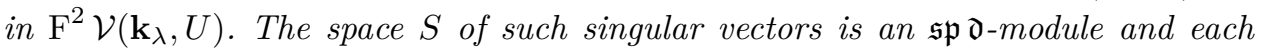
non-constant $\mathfrak{s p} \mathfrak{d}$-irreducible summand lying in $\mathrm{F}^{1} \mathcal{V}\left(\mathbf{k}_{\lambda}, U\right)$ generates an irreducible $\mathcal{P}$-module. The description of the subspaces of singular vectors is the same as in Proposition 11.1(i).

(ii) If the $\mathfrak{s p \mathfrak { d } - m o d u l e ~} U$ is infinite-dimensional irreducible, then $\mathcal{V}\left(\mathbf{k}_{\lambda}, U\right)$ does not contain non-constant singular vectors.

(iii) If a $\mathcal{P}$-module $\mathcal{V}\left(\mathbf{k}_{\lambda}, U\right)$ is not irreducible, then it decomposes as a direct sum of its two irreducible submodules.

\section{Acknowledgments}

This research was partially conducted during the authors' visits to the University of Rome La Sapienza, MIT, IHES and ETH. The first author was supported in part by a Simons Foundation grant 610284 . The second author was partially supported by Ateneo fundings from University of Rome La Sapienza grant RG120172B9BA78DF. The third author was partially supported by the Bert and Ann Kostant fund and by a Simons Fellowship grant 610284 . We are grateful to the referee for carefully reading the manuscript and for many valuable comments.

\section{References}

[1] B. Bakalov, A. D'Andrea, V.G. Kac, Theory of finite pseudoalgebras, Adv. Math. 162 (2001) 1-140.

[2] B. Bakalov, A. D'Andrea, V.G. Kac, Irreducible modules over finite simple Lie pseudoalgebras I. Primitive pseudoalgebras of type $W$ and S, Adv. Math. 204 (2006) 278-346.

[3] B. Bakalov, A. D'Andrea, V.G. Kac, Irreducible modules over finite simple Lie pseudoalgebras II. Primitive pseudoalgebras of type K, Adv. Math. 232 (2013) 188-237.

[4] A. Beilinson, V. Drinfeld, Chiral Algebras, AMS Colloquium Publications, vol. 51, American Math. Society, Providence, RI, 2004.

[5] A. D'Andrea, V.G. Kac, Structure theory of finite conformal algebras, Sel. Math. New Ser. 4 (3) (1998) 377-418.

[6] M. Eastwood, Extensions of the coeffective complex, Ill. J. Math. 57 (2) (2013) 373-381.

[7] W. Fulton, J. Harris, Representation Theory. A First Course, Readings in Mathematics, Graduate Texts in Mathematics, vol. 129, Springer-Verlag, New York, 1991.

[8] V.G. Kac, Vertex Algebras for Beginners, University Lecture Series, vol. 10, American Math. Society, Providence, RI, 1996, 2nd edition, 1998.

[9] A.L. Onishchik, E.B. Vinberg, Lie Groups and Algebraic Groups, Springer Series in Soviet Mathematics, Springer-Verlag, Berlin, 1990.

[10] A.N. Rudakov, Irreducible representations of infinite-dimensional Lie algebras of types S and H, Izv. Akad. Nauk SSSR, Ser. Mat. 39 (1975) 496-511 (in Russian); English translation in Math. USSR, Izv. 9 (1976) 465-480.

[11] M. Rumin, Formes différentielles sur les variétés de contact, J. Differ. Geom. 39 (1994) $281-330$.

[12] M. Sweedler, Hopf Algebras, Math. Lecture Note Series, W. A. Benjamin, Inc., New York, 1969. 